\title{
Alternative Considerátions for Environmental Oversight Training: Results from a Needs Assessment
}

Environmental Assessment Division Argonne National Laboratory

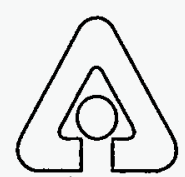

Operated by The University of Chicago, under Contract W-31-109-Eng-38, for the

United States Department of Energy

DISTRIBUTION OF THIS DOCUMENT IS UNLIMITED 


\section{Argonne National Laboratory}

Argonne National Laboratory, with facilities in the states of Illinois and Idaho, is owned by the United States Government, and operated by the University of Chiçago under the provisions of a contract with the Department of Energy.

This technical memo is a product of Argonne's Environmental Assessment Division (EAD). For information on the division's scientific and engineering activities, contact:

Director, Environmental Assessment Division

Argonne National Laboratory

Argonne, Illinois 60439-4815

Telephone (708) 252-3107

Presented in this technical memo are preliminary results of ongoing work or work that is more limited in scope and depth than that described in formal reports issued by the EAD."

Publishing support services were provided by Argonne's Information and Publishing Division (for more information, see IPD's home page: http://www.ipd.anl.gov/).

\section{Disclaimer}

This report was prepared as an account of work sponsored by an agency of the United States Government. Neither the United States Government nor any agency thereof, nor any of their employees, makes any warranty, express or implied, or assumes any legal liability or responsibility for the accuracy, completeness, or usefulness of any information, apparatus, product, or process disclosed, or represents that its use would not infringe privately owned rights. Reference herein to any specific commercial product, process, or service by trade name, trademark, manufacturet, or otherwise, does not necessarily constitute or imply its endorsement, recommendation, or favoring by the United States Government or any agency thereof. The views and opinions of authors expressed herein do not necessarily state or reflect those of the. United States Government or any agency thereof.

Reproduced directly from the best available copy.

Available to DOE and DOE contractors from the Office of Scientific and Technical Information, P.O. Box 62, Oak Ridge, TN-37831; prices available from (423) 576-8401.

Available to the public from the National Technical . 5285 Port Royal Road, Springfield, VA 22161. 


\section{Alternative Considerations for Environmental Oversight Training: Results from a Needs Assessment}

by C. Young and J. Hensley

November 1995

Work sponsored by the U.S. Department of Energy,

Office of Northwestern Area Programs, Washington, D.C.

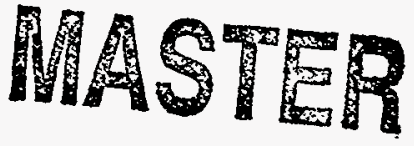

do

DISTRIBUTION OF THIS DOCUMENT IS UNLIMITED 
This report is printed on recycled paper. 


\section{CONTENTS}

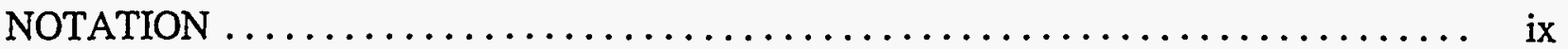

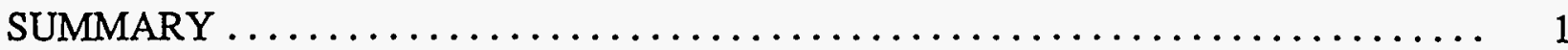

1 INTRODUCTION $\ldots \ldots \ldots \ldots \ldots \ldots \ldots \ldots \ldots \ldots \ldots \ldots \ldots \ldots \ldots \ldots \ldots \ldots \ldots$

2 METHODS $\ldots \ldots \ldots \ldots \ldots \ldots \ldots \ldots \ldots \ldots \ldots \ldots \ldots \ldots \ldots \ldots \ldots \ldots$

2.1 Workbook $\ldots \ldots \ldots \ldots \ldots \ldots \ldots \ldots \ldots \ldots \ldots \ldots \ldots \ldots \ldots \ldots \ldots \ldots \ldots$

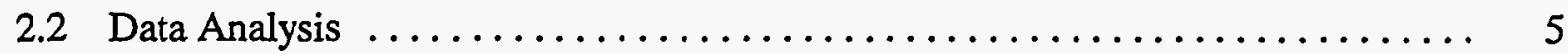

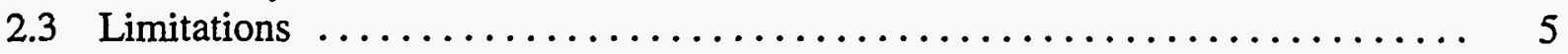

3 RESULTS $\ldots \ldots \ldots \ldots \ldots \ldots \ldots \ldots \ldots \ldots \ldots \ldots \ldots \ldots \ldots \ldots \ldots \ldots$

3.1 Tasks Performed $\ldots \ldots \ldots \ldots \ldots \ldots \ldots \ldots \ldots \ldots \ldots \ldots \ldots \ldots \ldots \ldots$

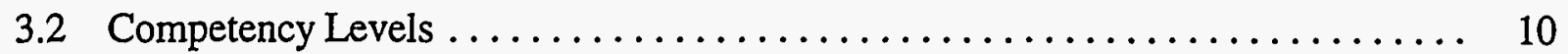

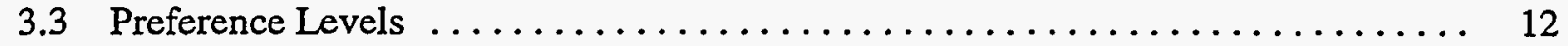

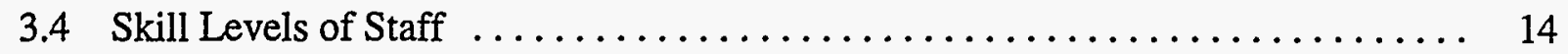

4 CONCLUSIONS $\ldots \ldots \ldots \ldots \ldots \ldots \ldots \ldots \ldots \ldots \ldots \ldots \ldots \ldots \ldots \ldots \ldots$

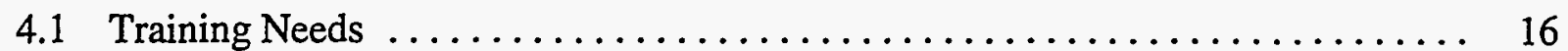

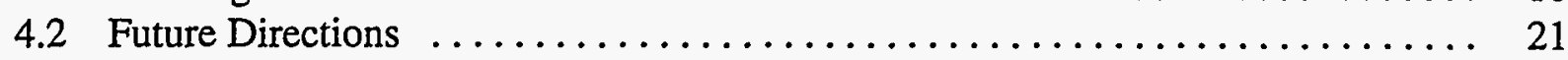

4.2 .1 Develop Training Activities ...................... 21

4.2 .2 Evaluate the Effectiveness of Training Activities . . . . . . . . . . 22

4.2.3 Demonstrate Management's Commitment .................. 22

4.2 .4 Explore Related Data Applications . . . . . . . . . . . . . . . . . . 23

4.2 .5 Apply Methodology to Other Offices ................... 23

5 REFERENCES $\ldots \ldots \ldots \ldots \ldots \ldots \ldots \ldots \ldots \ldots \ldots \ldots \ldots \ldots \ldots \ldots \ldots \ldots \ldots$

APPENDIX A: Data Collection Workbook ..................... A-1

APPENDIX B: Most Critical Skills for Each Task .................. B-1

APPENDIX C: Skills with Highest and Lowest Average Scores $\ldots \ldots \ldots \ldots \ldots \ldots \ldots$ C-1 


\section{TABLES}

1 Percentage of Tasks that Staff Perform and Should Perform $\ldots \ldots \ldots \ldots \ldots \ldots$

2 Tasks that Staff Generally Do Not and Should Not Perform $\ldots \ldots \ldots \ldots \ldots$

3 Common Skills for Tasks that Most Staff Perform and Should Perform ......... 10

4 Tasks that Staff Perform Relatively Less Well $\ldots \ldots \ldots \ldots \ldots \ldots \ldots \ldots \ldots$

5 Common Skills for Tasks with the Lowest Competency Levels .............. 11

6 Tasks with Relatively Wide Ranges of Ability $\ldots \ldots \ldots \ldots \ldots \ldots \ldots \ldots \ldots$

7 Common Skills Associated with Tasks with Relatively

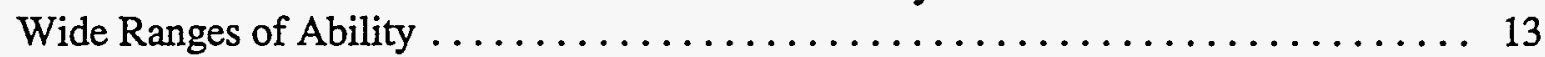

8 Most Preferred and Least Preferred Tasks that Staff Perform ............... 13

9 Common Skills Associated with Most Preferred Tasks that Staff Perform . . . . . . 14

10 Common Skills Associated with Least Preferred Tasks that Staff Perform ........ 14

11 Common Skills Associated with Various Sets of Tasks .................. 17

12 Priority Ranking for 17 Common Skills for which Training

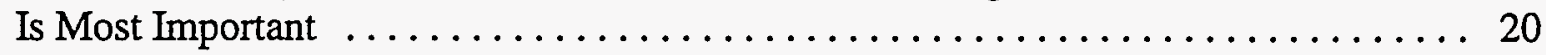

B.1 Most Critical Skills Needed to Prepare Documents $\ldots \ldots \ldots \ldots \ldots \ldots \ldots \ldots$ B-3

B.2 Most Critical Skills Needed to Review Documents $\ldots \ldots \ldots \ldots \ldots \ldots \ldots$ B-3

B.3 Most Critical Skills Needed to Coordinate Document Reviews $\ldots \ldots \ldots \ldots \ldots \ldots$. B-4

B.4 Most Critical Skills Needed to Develop Testimony Questions

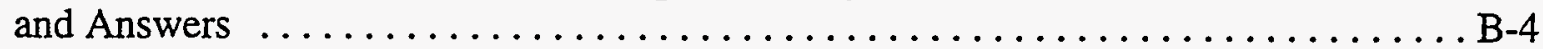

B.5 Most Critical Skills Needed to Conduct Briefings/Presentations $\ldots \ldots \ldots \ldots \ldots \ldots$. -5

B.6 Most Critical Skills Needed to Interface with DOE $\ldots \ldots \ldots \ldots \ldots \ldots \ldots$ B-6

B.7 Most Critical Skills Needed to Advise, Assist, and Provide

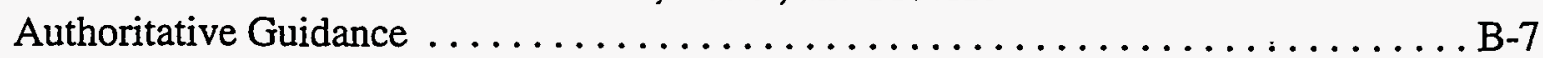




\section{TABLES (Cont.)}

B.8 Most Critical Skills Needed to Provide Regulatory Analysis

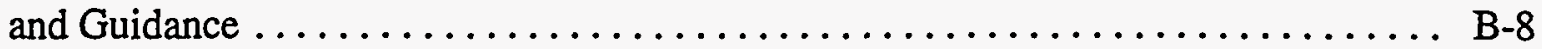

B.9 Most Critical Skills Needed to Implement Regulations and Policy ........... B-9

B.10 Most Critical Skills Needed to Provide Support to Conduct

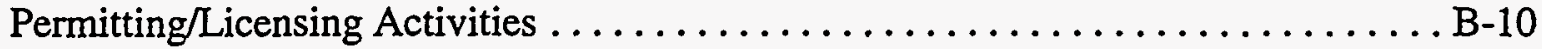

B.11 Most Critical Skills Needed to Operate Equipment $\ldots \ldots \ldots \ldots \ldots \ldots \ldots \ldots$.

B.12 Most Critical Skills Needed to Manage Files and Records ............... B-12

B.13 Most Critical Skills Needed to Track Correspondence ................. B-13

B.14 Most Critical Skills Needed to Perform Travel Administration Functions ......... B-14

B.15 Most Critical Skills Needed to Participate in Personnel

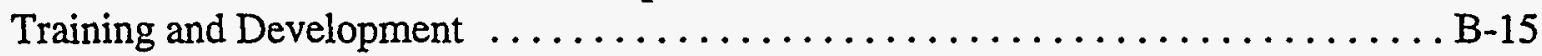

B.16 Most Critical Skills Needed to Oversee Data/Information Management ......... B-16

B.17 Most Critical Skills Needed to Address Personnel/Staffing Issues . . . . . . . . . . B-17

B.18 Most Critical Skills Needed to Track and Verify Corrective Actions ........... B-18

B.19 Most Critical Skills Needed to Oversee Surveillance/

Monitoring/Inspection Activities ............................ B-19

B.20 Most Critical Skills Needed to Investigate Complaints,

Allegations, and Incidents $\ldots \ldots \ldots \ldots \ldots \ldots \ldots \ldots \ldots \ldots \ldots \ldots \ldots \ldots \ldots \ldots \ldots \ldots \ldots \ldots$

B.21 Most Critical Skills Needed to Provide Analyses to Determine

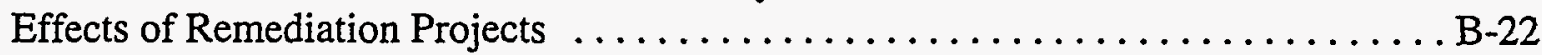

B.22 Most Critical Skills Needed to Review Remediation

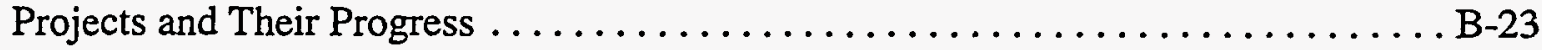

B.23 Most Critical Skills Needed to Recommend Actions to

Correct Remedial Problems . . . . . . . . . . . . . . . . . . . . . . . B-24

B.24 Most Critical Skills Needed to Identify and Document Deficiencies $\ldots \ldots \ldots \ldots$ B-25

B.25 Most Critical Skills Needed to Set Schedules $\ldots \ldots \ldots \ldots \ldots \ldots \ldots \ldots \ldots$. B-26 


\section{TABLES (Cont.)}

B.26 Most Critical Skills Needed to Identify and Conduct Facility Acceptance ....... B-27

B.27 Most Critical Skills Needed to Oversee Assessments for Cleanup ............ B-28

B.28 Most Critical Skills Needed to Oversee Environmental

Restoration Activities

B.29 Most Critical Skills Needed to Oversee Waste Management Activities ......... B-30

B.30 Most Critical Skills Needed to Oversee Toxic and Hazardous

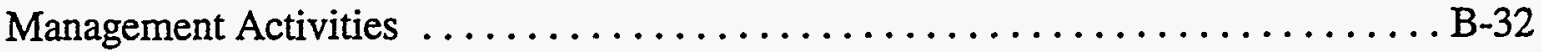

B.31 Most Critical Skills Needed to Oversee Water Quality Activities ............ B-34

B.32 Most Critical Skills Needed to Oversee Air Quality Activities ............... B-35

B.33 Most Critical Skills Needed to Oversee Cultural Resources

Management Activities $\ldots \ldots \ldots \ldots \ldots \ldots \ldots \ldots \ldots \ldots \ldots \ldots \ldots \ldots \ldots \ldots \ldots \ldots$

B.34 Most Critical Skills Needed to Oversee Radiation Protection Activities ......... B-37

B.35 Most Critical Skills Needed to Oversee NEPA Activities $\ldots \ldots \ldots \ldots \ldots \ldots$. . . . 39

B.36 Most Critical Skills Needed to Oversee Decontamination and Decommissioning $\ldots \ldots \ldots \ldots \ldots \ldots \ldots \ldots \ldots \ldots \ldots \ldots \ldots \ldots \ldots \ldots \ldots$

B.37 Most Critical Skills Needed to Oversee Quality Assurance and

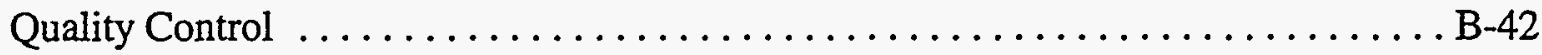

B.38 Most Critical Skills Needed to Oversee Land Use Activities ................ B-44

B.39 Most Critical Skills Needed to Evaluate Alternatives for Cleanup ............ B-45

B.40 Most Critical Skills Needed to Oversee Program Evaluations $\ldots \ldots \ldots \ldots \ldots$. . . 46

B.41 Most Critical Skills Needed to Manage Contracts $\ldots \ldots \ldots \ldots \ldots \ldots \ldots \ldots$. B-47

B.42 Most Critical Skills Needed to Evaluate Contractors $\ldots \ldots \ldots \ldots \ldots \ldots \ldots$ B-48

B.43 Most Critical Skills Needed to Oversee Independent Verification $\ldots \ldots \ldots \ldots \ldots$. B-49

B.44 Most Critical Skills Needed to Identify and Allocate Resources $\ldots \ldots \ldots \ldots \ldots$. B-51 


\section{TABLES (Cont.)}

B.45 Most Critical Skills Needed to Provide Goods and Services $\ldots \ldots \ldots \ldots \ldots$. . 52

B.46 Most Critical Skills Needed to Oversee Cost Reviews ................. B-54

B.47 Most Critical Skills Needed to Formulate, Defend, and Modify Budget ........ B-56

B.48 Most Critical Skills Needed to Develop, Coordinate, and Disseminate Program Management Policies and Procedures .............. B-57

B.49 Most Critical Skills Needed to Establish Cost Schedule and Technical Baselines . . . . . . . . . . . . . . . . . . . . . . . . . . 58

B.50 Most Critical Skills Needed to Develop Resource Requirements . . . . . . . . . . . B-59

B.51 Most Critical Skills Needed to Develop New/Improve Technologies .......... B-60

B.52 Most Critical Skills Needed to Oversee Research, Development, Demonstration, Testing, and Evaluation Activities .................. B-62

C.1 10 Highest Average Scores on General Knowledge/Skills $\ldots \ldots \ldots \ldots \ldots \ldots$ C-3

C.2 10 Lowest Average Scores on General Knowledge/Skills $\ldots \ldots \ldots \ldots \ldots \ldots$ C-4

C.3 10 Highest Average Scores on Technical Knowledge $\ldots \ldots \ldots \ldots \ldots \ldots \ldots$ C-5

C.4 10 Lowest Average Scores on Technical Knowledge $\ldots \ldots \ldots \ldots \ldots \ldots \ldots$ C-6

C.5 10 Highest Average Scores on Regulatory Knowledge $\ldots \ldots \ldots \ldots \ldots \ldots \ldots$ C-7

C.6 10 Lowest Average Scores on Regulatory Knowledge $\ldots \ldots \ldots \ldots \ldots \ldots \ldots$ C-8 


\section{NOTATION}

The following is a list of the acronyms, initialisms, and abbreviations used in this document.

\section{ACRONYMS, INITIALISMS, AND ABBREVIATIONS}

CERCLA Comprehensive Environmental Response, Compensation, and Liability Act

D\&D Decontamination and Decommissioning

DOE U.S. Department of Energy

EM Office of Environmental Management

IDP Individual Development Plan

NEPA National Environmental Policy Act

RCRA Resource Conservation and Recovery Act

RDDT\&E Research, Development, Demonstration, Testing, and Evaluation 


\title{
ALTERNATIVE CONSIDERATIONS FOR ENVIRONMENTAL OVERSIGHT TRAINING: RESULTS FROM A NEEDS ASSESSMENT
}

by

\author{
C. Young and J. Hensley
}

SUMMARY

A headquarters organization of the U.S. Department of Energy's (DOE's) Office of Environmental Management directs and oversees environmental remediation activities at the Chicago, Idaho, Oakland, and Richland Operations Offices. To successfully oversee environmental remediation activities, staff must be adequately trained in the various tasks they perform. Argonne National Laboratory conducted a training needs assessment for this headquarters organization by having staff members identify tasks performed and the knowledge, skills, and abilities needed to accomplish those tasks. This information provided the basis for determining and prioritizing possible training needs.

As part of the training needs assessment, staff from this headquarters office completed a workbook to identify the most critical skills needed for each task. Respondents also identified tasks they actually perform and should perform. These tasks are listed in staff's position descriptions, assigned by their supervisors, or based on DOE policy. Staff were also asked to specify the tasks they do not perform and their preferences for performing each task. Further, staff rated their competency in performing each task and how appropriate they perceived their knowledge and skills to be.

Results from the assessment suggest that staff from this office perform diverse tasks that they should perform and that they generally like performing. Staff identified 10 tasks that should be performed by an additional $10 \%$ of staff. These tasks related to functions such as program oversight, document preparation, and policy analysis. Staff have relatively wide ranges of ability for some of the tasks that they currently perform and should perform. Moreover, those tasks that most staff do not or should not perform are generally those tasks that are least preferred.

The results suggest staff are moderately proficient in using the skills needed to perform the above tasks. However, for the organization to function at its best, staff must be extremely proficient in the skills they use to carry out most of their tasks. Seventeen important, common skills were found to be associated with the multiple tasks that staff perform and should perform. Given the importance of these skills, training is appropriate. These 17 skills fall into three broad categories: (1) communication, (2) critical thinking/problem solving, and (3) program management. Examples include "writing clearly and succinctly"; "assessing strengths and weaknesses of alternatives"; and 
knowledge of "planning, programming, funding, and acquisition management." Staff should be encouraged to attend training in these areas.

Next steps include improving the knowledge and skills of staff of this headquarters office, particularly in the 17 common skills necessary for most tasks that staff perform and should perform. This improvement can be accomplished by identifying and examining different types of skill development opportunities to accomplish a high proficiency level. Not only should formal training courses be identified or developed, but on-the-job experiences should also be considered. The opportunities for training should then be evaluated for their effectiveness to determine which training is most useful. Management support for training should be demonstrated through actions such as developing and carrying out plans that address individual training needs and professional development. 


\section{INTRODU்CTION}

For staff to perform their jobs effectively and efficiently, they must be adequately trained. Well-trained staff are also more likely to be satisfied with their jobs and to remain with a given organization (Kleiman 1993; Hubble and Green 1992). In addition to hiring staff with relevant backgrounds and skills, critical steps in maintaining adequately trained staff are to analyze skill levels needed for the various tasks that personnel are required to perform and to provide training to improve staff's skill base. This first analysis is commonly referred to as a training needs assessment. Training needs are usually determined by defining the tasks required for a particular job and the associated knowledge, skills, and abilities necessary to adequately accomplish these tasks (Cook and Seith 1992; Marshall and Mayer 1992).

The Office of Northwestern Area Programs of the U.S. Department of Energy's (DOE's) Office of Environmental Management (EM) oversees environmental remediation activities in the Chicago, Idaho, Oakland, and Richland Operations Offices. For this organization to effectively carry out its mission, its staff need to be as proficient as possible in the appropriate knowledge and skills. Therefore, a training needs assessment was conducted to determine staff's level of knowledge and proficiency in various skills. The purpose of the assessment was to:

- Examine the types of activities or tasks in which staff are involved,

- Determine the skills needed to perform relevant tasks, and

- Assess gaps in knowledge and skills for the tasks performed in order to suggest opportunities for skill development. 


\section{METHODS}

This section describes the methods used to gather and analyze information to determine where training would be useful.

\subsection{WORKBOOK}

A workbook was designed to collect information in three major areas: tasks that staff perform and believe they should perform, skills ${ }^{1}$ associated with these tasks, and staff members' own assessments of their levels of expertise in these skills. These areas are standard components of a training needs assessment (Birnbrauer and Tyson 1985; Fisher and Frank 1992; Nadler 1982; Zemke 1985).

To prepare the workbook, information was gathered from multiple sources to identify the range of tasks performed. First, position descriptions were reviewed to generate a list of possible tasks performed by staff. Because position descriptions are not updated as frequently as job functions evolve and, therefore, may not accurately reflect the activities actually performed, additional sources were solicited. Additional lists of tasks for environmental programs were obtained from the DOE Office of Professional and Technical Training and Development, from the Office of Oversight and Self-Assessment, and from previous investigations into environmental activities in other agencies (Close 1987; Meshenberg et al. 1990). Tasks were consolidated to reduce duplication and to create consistency across a wide range of science and engineering position types. These tasks related to broad organizational functions such as communication, administration, personnel, project management, project implementation, remedial activities, and budget.

Information on the underlying knowledge and skills necessary to complete the indicated tasks was also obtained from multiple sources, including position descriptions, job announcements, and literature on environmental training, problem solving, and decision making (Green et al. 1985; Posner 1989; Segal et al. 1985). Additional information was obtained from the Office of Professional and Technical Training and Development, from previous work performed by the Office of Compliance and Program Coordination, and from performance evaluation criteria of this headquarters office. The lists of knowledge and skills were also consolidated to reduce duplication. Skills were listed under the broad categories of communication, administration, organizational structure and process, supervision, physical sciences, engineering, safety and health, biology, management of hazardous materials and wastes, federal environmental laws and regulations, DOE orders, and executive orders on environmental topics.

1 The term skills is used throughout the text and tables to refer to skills, knowledge, and/or abilities. 
The lists of tasks and skills were then arranged into a pilot version of the workbook that contained three parts with accompanying instructions. Part 1 contained questions about activities or tasks in which staff are involved. Staff were asked which tasks they perform and should perform. This category was defined as consisting of tasks listed in their position descriptions, assigned by their supervisors, or based on DOE policy. Staff were also asked how much they liked performing these tasks and how well they thought they performed them. Part 2 asked the staff to identify the knowledge and skills needed to perform the tasks in Part 1. Part 3 addressed the individual staff member's level of proficiency with different types of knowledge and skills. Four staff members and training experts at Argonne National Laboratory reviewed the pilot version of the workbook. Their review focused on clarity of instructions, workbook format, completeness of task and skill lists, and ease of responding to questions. Reviewers indicated that definitions of tasks and skills were unnecessary but suggested the lists of regulatory knowledge be expanded.

A final workbook (see Appendix A) was then prepared and distributed to all 23 staff members. All workbooks were returned; 14 individuals completed all three sections, and the remaining staff completed parts of the workbook. A master code book was compiled, and the responses were entered into a database in Excel (Microsoft Corporation 1992).

\subsection{DATA ANALYSIS}

Responses were tabulated in Excel for each section of the workbook. For Part 1 (Tasks), the following were computed: (1) the proportion of staff that perform and should perform each task, (2) average score of staff's competency to perform each task, and (3) average score on levels of preference for tasks. For Part 2 (Ideal Skills for Each Task), the mean level of importance of all skills for each task was calculated. These mean scores were then sorted in descending order to determine the most important skills needed for each task. Appendix B of this report presents the skills that received the 10 highest mean criticality scores for each task. For Part 3 (Skills), the average score of competency of staff members' skill levels was calculated for each skill.

\subsection{LIMITATIONS}

The analysis provides valuable information on tasks associated with oversight of environmental remediation and skill levels of staff; the results also may be used to suggest training needs. However, the study has limitations. The response rate to the three parts of the workbook was variable. Part 2 of the workbook, which linked skills to tasks, was completed by only 14 of the 23 staff members.

In terms of data analysis, mean scores are presented in the aggregate for all staff. As a result, it is impossible to obtain information on skills associated with a task that only one person may perform or a skill that only one person may need. 
In addition, the approach used in this project was a process of self-assessment - a standard approach for conducting needs assessments (Kosecoff and Fink 1982; American Society for Training and Development 1990). Staff indicated whether they performed various tasks, how well they performed those tasks, and their own skill level. Although these perceptions are valuable, they offer only one point of view. Another useful perspective could be managers' assessments of relevant tasks and skill levels of staff. (These perspectives have been solicited, and the results corroborate those presented in this report [Young and Hensley 1995; Hensley and Young 1995].) Even the perspectives of "outsiders" could be included - for example, evaluations by English teachers of staff writing samples.

Furthermore, despite pilot testing and review by experts, the lists of skills and tasks may not be exhaustive or may not be mutually exclusive. 


\section{RESULTS}

\subsection{TASKS PERFORMED}

In Part 1 of the workbook, staff were asked to specify whether they currently perform a variety of tasks and whether they should perform tasks that they currently do not. The purpose of these questions was to identify activities or tasks that staff perform on a fairly regular basis, as well as to identify those that are irrelevant to the function of this headquarters organization.

Tasks that were considered important for this training needs assessment are those that most of the staff perform and feel they should perform as part of their job responsibilities. Tasks that staff should perform are those listed in their position descriptions, assigned by their supervisors, or based on DOE policy. As expected, most staff members perform and feel they should perform diverse types of tasks (Table 1). Tasks that most staff perform are related to the broad functions of preparing and processing documents, performing administrative/management duties, implementing guidance, and carrying out general remedial activities; they are not discipline-specific (e.g., "monitoring air quality"). Examples of these diverse tasks include "coordinate document review," "set schedules," and "implement regulations and policy." Moreover, respondents indicated that the tasks they are currently not performing (Table 2) and those that they should not perform are nearly identical.

For the most part, staff members believe they should perform the tasks they are currently performing. ${ }^{2}$ However, staff identified 10 tasks that should be performed by at least an additional $10 \%$ of staff (Table 1). For example, although most staff "formulate, defend, and modify budget," an additional $14 \%$ of the staff indicated they should perform this task. This finding suggests a rather large gap in the proportion of staff who perform this task relative to the proportion of those who should perform it. Therefore, skill development opportunities may be appropriate.

The following tasks should be performed by an additional $10 \%$ of staff:

- "Conduct briefings/presentations";

- “Oversee assessments for cleanup";

- "Provide analyses to determine effects of remediation projects";

- "Evaluate contractors";

- "Develop testimony questions and answers";

2 Hereafter, "tasks that staff perform and should perform" will be simplified to "tasks that staff perform" (because the tasks that staff perform and should perform are essentially identical). 
TABLE 1 Percentage ${ }^{a}$ of Tasks that Staff Perform and Should Perform

\begin{tabular}{|c|c|c|}
\hline Task & $\begin{array}{l}\text { Perform } \\
(\%)\end{array}$ & $\begin{array}{l}\text { Should } \\
\text { Perform } \\
\text { (\%) }\end{array}$ \\
\hline Advise, assist, and provide authoritative guidance ${ }^{b}$ & 90 & 100 \\
\hline Coordinate document reviews (in/out DOE) & 100 & 100 \\
\hline Review documents & 100 & 100 \\
\hline Formulate, defend, and modify budget ${ }^{b}$ & 80 & 100 \\
\hline Implement regulations and policy & 90 & 100 \\
\hline Interface within/outside DOE & 90 & 100 \\
\hline Conduct briefings/presentations ${ }^{b}$ & 70 & 90 \\
\hline Identify and document deficiencies & 90 & 90 \\
\hline Operate equipment & 100 & 90 \\
\hline Oversee environmental restoration activities & 80 & 90 \\
\hline Provide analyses to determine effects of remediation projects ${ }^{b}$ & 50 & 90 \\
\hline Review remediation projects and their progress & 90 & 90 \\
\hline Oversee assessments for cleanup $p^{b}$ & 60 & 90 \\
\hline Evaluate alternatives for cleanup & 80 & 90 \\
\hline Develop testimony questions and answers ${ }^{b}$ & 70 & 80 \\
\hline Recommend actions to correct remedial problems & 80 & 80 \\
\hline Provide regulatory analysis and guidance & 80 & 80 \\
\hline Evaluate contractors ${ }^{\mathrm{b}}$ & 50 & 80 \\
\hline $\begin{array}{l}\text { Develop, coordinate, and disseminate program management } \\
\text { policies and procedures }\end{array}$ & 70 & 80 \\
\hline Oversee cost reviews ${ }^{b}$ & 70 & 80 \\
\hline Oversee National Environmental Policy Act (NEPA) activities & 80 & 80 \\
\hline Prepare documents & 80 & 80 \\
\hline Oversee program evaluations ${ }^{b}$ & 60 & 80 \\
\hline Establish cost, schedule, and technical baselines & 70 & 70 \\
\hline Track and verify corrective actions & 70 & 70 \\
\hline Set schedules & 70 & 70 \\
\hline Participate in personnel training and development ${ }^{b}$ & 50 & 70 \\
\hline Oversee quality assurance/quality control activities & 60 & 60 \\
\hline $\begin{array}{l}\text { Oversee data/information management (e.g., Occurrence } \\
\text { Reporting and Processing System) }\end{array}$ & 70 & 60 \\
\hline Manage files and records & 80 & 50 \\
\hline
\end{tabular}

a Percentages have been rounded to the nearest $10 \%$ to simplify rankings.

b At least an additional $10 \%$ of staff should perform this task. 
TABLE 2 Tasks that Staff Generally Do Not and Should Not Perform ${ }^{\mathrm{a}}$

Oversee air quality activities

Oversee land use activities

Perform travel administrative functions

Investigate complaints, allocations, and incidents

Develop new/improved technologies

Oversee RDDT\&E ${ }^{\mathrm{b}}$ activities

Manage contracts

Procure goods and services

Track correspondence

a Tasks that less than $30 \%$ of staff perform.

b Research, development, demonstration, testing, and evaluation.

- "Advise, assist, and provide authoritative guidance";

- "Formulate, defend, and modify budget";

- "Oversee cost reviews";

- “Oversee program evaluations"; and

- "Participate in personnel training and development."

Once the relevant tasks were identified from the complete list of tasks in the workbook, the underlying skills needed to carry out these tasks were determined. Skills needed for multiple tasks were termed "common skills." Common skills are those among the five most critical for a given task and needed for at least $30 \%$ of the relevant set of tasks. All staff should be proficient in these skills. Several common skills were found to be associated with the tasks that most staff perform (Table 3); examples include writing well, "keeping abreast of current developments," and "assessing strengths and weaknesses of alternatives."

The common skills associated with those tasks that an additional $10 \%$ of staff should perform are similar to those common skills associated with those tasks that most staff currently perform (Table 3). For the common skills discussed so far, staff indicated they have a working knowledge of these skills, although very few consider themselves experts in any of them. 


\section{TABLE 3 Common Skills ${ }^{\mathrm{a}}$ for Tasks that Most Staff Perform and Should Perform}

Organizing/categorizing

Assessing strengths and weaknesses of alternatives

Organizing information and presenting cogent arguments

Prioritizing based on criteria

Keeping abreast of current developments ${ }^{b}$

Providing clear guidance and direction to accomplish tasks ${ }^{b}$

Writing succinctly and clearly

Examining trade-offs among alternatives

Addressing schedule constraints

Identifying relevant material/requirements ${ }^{\mathrm{b}}$

Defining applicable criteria ${ }^{b}$

EM's mission, policies, management plans, procedures, priorities, goals, and objectives ${ }^{b}$

DOE management strategies

a Skills that were among the top 5 most critical skills for at least 9 of the 30 tasks that most staff perform. These skills have a mean score ranging from 3.1 at top of table to 2.4 at bottom of table based on a 4-point scale, where $1=$ no knowledge;

2 = familiarity; 3 = working knowledge; and 4 = highly proficient/expert.

b Common skills for tasks that at least $10 \%$ more staff should perform.

\subsection{COMPETENCY LEVELS}

Staff were asked to rate on a 4-point scale how well they perform each task, with 1 being a low proficiency level and 4 being a high proficiency level. The purpose of this question was to determine whether there are tasks that staff do not perform well and to examine whether staff had widely varying ability levels in performing particular tasks. For those tasks that staff perform, high mean scores, low mean scores, and widely varying mean scores on competency were examined.

Those tasks for which staff have the lowest (although still generally moderate) competency levels include "identify and document deficiencies"; "manage files and records"; and oversight related to cleanup, cost reviews, National Environmental Policy Act (NEPA) activities, and data and information management (Table 4). Except for three common skills, staff use the same common skills to perform tasks regardless of whether they perform these tasks well or less well (Table 5). These three exceptions are "project or program management techniques," "analyzing information," and "knowledge of interagency agreements, administration, and process." 
TABLE 4 Tasks that Staff Perform

Relatively Less Well ${ }^{\mathrm{a}}$

Implement regulations

Oversee assessments for cleanup

Oversee cost reviews

Oversee NEPA activities

Provide regulatory analysis and guidance

Oversee data/information management

Evaluate contractors

Manage files and records

Identify and document deficiencies

Oversee quality assurance/quality control

a Tasks with a mean score ranging from 2.9 at top of table to 2.7 at bottom of table based on a 4-point scale, where $1=$ not at all well; 2 = somewhat well; 3 = sufficiently well; and $4=$ extremely well.

TABLE 5 Common Skills ${ }^{\text {a }}$ for Tasks with the Lowest Competency Levels

Interagency agreements, administration, and process

Providing clear guidance and direction to accomplish tasks

EM's mission, policies, management plans, procedures, priorities, goals, and objectives

Keeping abreast of current developments

Organizing/categorizing

Addressing schedule constraints

Assessing strengths and weaknesses of alternatives

Identifying relevant materials/requirements

Defining applicable criteria

Project or program management techniques

Analyzing information

Examining trade-offs among alternatives

a Skills that apply to at least 4 of the 10 tasks that staff do least well. 
Staff were found to have relatively wide ranges of ability for several of the tasks they perform (Table 6). Rather than falling easily into categories, these tasks were quite diverse. Examples include "conduct briefings and presentations," "oversee assessments for cleanup," "evaluate contractors," and "oversee cost reviews."

All but three common skills for tasks for which staff have relatively wide-ranging ability (Table 7) are the same as the common skills for tasks that staff perform (Table 3). These three exceptions are "project and program management techniques"; "understanding schedule constraints"; and "planning, programming, funding, and acquisition management."

\subsection{PREFERENCE LEVELS}

Staff were asked to indicate the desirability of the tasks they perform. The rationale underlying this question was to ascertain those tasks that staff might easily choose and those tasks that might be burdensome and, therefore, less likely to be accomplished. If staff are provided with the opportunity to improve their skill proficiency for least preferred tasks, these tasks may become more desirable. The tasks with the highest and lowest mean scores on desirability/preference were examined.

Staff indicated that seven tasks were considered highly desirable (Table 8). These primarily relate to technical functions such as remediation activities and assessments. On the other hand, five tasks were considered undesirable, particularly those related to reporting ("develop testimony questions and answers," "prepare documents," and "coordinate document reviews"). Staff members also considered undesirable the task of overseeing NEPA activities because it duplicates other activities, particularly those associated with the Comprehensive Environmental Response, Compensation, and Liability Act (CERCLA). Tasks associated with NEPA were also viewed as having a subjective approval process and ambiguous guidance.

TABLE 6 Tasks with Relatively Wide Ranges ${ }^{\text {a }}$ of Ability

Develop, coordinate, and disseminate program management policies

Participate in personnel training and development

Conduct briefings and presentations

Oversee NEPA activities

Oversee quality assurance/quality control activities

Oversee assessments for cleanup

Oversee cost reviews

Evaluate contractors

a Standard deviation, or the amount of variability around the mean ability score, is $\geq 0.75$. 
TABLE 7 Common Skills ${ }^{\text {a }}$ Associated with Tasks with Relatively Wide Ranges of Ability

Providing clear guidance and direction to accomplish tasks

DOE management strategies

EM's mission, policies, management plans, procedures, priorities, goals, and objectives

Keeping abreast of current developments

Project or program management techniques

Addressing schedule constraints

Planning, programming, funding, and acquisition management

Identifying relevant material/requirements

Defining applicable criteria

a Skills that apply to at least four of the eight tasks with relatively wide ranges of ability.

TABLE 8 Most Preferred and Least Preferred Tasks that Staff Perform

\section{Most Preferred Tasks}

Review remediation projects and their progress

Recommend actions to correct remedial problems

Evaluate alternatives for cleanup

Advise, assist, and provide authoritative guidance

Oversee environmental restoration (CERCLA) activities

Oversee assessments for cleanup

Interface within/outside of DOE

\section{Least Preferred Tasks}

Develop testimony questions and answers

Oversee NEPA activities

Prepare documents

Coordinate document review

Oversee quality assurance/quality control activities 
As described above, tasks with different preference levels were identified. The common skills associated with these tasks were then determined. The common skills associated with the most preferred tasks are listed in Table 9. The common skills associated with the least preferred tasks (Table 10) are similar to the common skills associated with all the tasks that staff perform (Table 3). These general skills include keeping abreast of current developments, organizing, writing clearly, and identifying relevant requirements.

\subsection{SKILL LEVELS OF STAFF}

Once the critical skills were identified for the tasks that staff perform, staff members' levels of proficiency with these various skills were examined. Levels of proficiency were evaluated for three categories of skills: general knowledge and skills (e.g., organizing information, assessing strengths and weaknesses); discipline-specific knowledge (e.g., chemistry, hazardous waste characteristics); and regulatory knowledge (e.g., federal laws, DOE orders).

TABLE 9 Common Skills ${ }^{\mathrm{a}}$ Associated with Most Preferred Tasks that Staff Perform

Organizing information and presenting cogent arguments

Providing clear guidance and direction to accomplish tasks

EM's mission, policies, management plans, procedures, priorities, goals, and objectives

Remediation techniques

RCRA ${ }^{b} / C E R C L A$ process

a Skills that apply to at least four of the seven most preferred tasks.

b Resource Conservation and Recovery Act.

TABLE 10 Common Skills $^{a}$ Associated with Least Preferred Tasks that Staff Perform

Writing clearly and succinctly

EM's mission, policies, management plans, procedures, priorities, goals, and objectives

Keeping abreast of current developments

Identifying relevant requirements

a Skills that apply to at least three of the five least preferred tasks. 
For the three categories of skills identified above, the skills for which staff have low abilities are those skills for which training is especially important. However, in general, staff indicated that their skill levels are moderate to good. Appendix $\mathrm{C}$ provides details for those skills with both high and low levels of competency. Although staff collectively indicated they have working knowledge of most skills and although some individually indicated they are experts, staff do not collectively consider themselves experts in any skills. This is an interesting finding because these headquarters staff work in a complex environment where they provide guidance to the operations offices, interface with federal and state agencies, and defend programs - all of which depend on highly proficient environmental remediation skills. Despite staff's working knowledge, then, training is still appropriate for this headquarters office.

Examples of those general skills for which staff indicated the most proficiency include "organizing/categorizing information," "conducting library research," "operating computers," "assessing the strengths and weaknesses of alternatives," and "prioritizing based on criteria." Examples of general skills for which staff indicated the least proficiency include "speed reading" and "touch typing." However, these low-proficiency skills are not especially critical to the tasks that staff perform (Tables 3, 5, 7, and 10; Appendixes B and C).

Staff rated their abilities higher for general skills than for discipline-specific knowledge, which was rated higher than knowledge of regulations and agency orders. In terms of these discipline-specific skills, staff collectively indicated they were most knowledgeable of the principles of RCRA/CERCLA, NEPA, environmental sciences, and remediation techniques (with mean scores ranging from 2.6 to 2.9 on a 4-point scale, with 4 representing expert knowledge). On the whole, staff were least proficient at geomapping techniques, cartography, and nuclear engineering. In their responses for regulatory knowledge, staff indicated they were most knowledgeable in the following regulations: CERCLA, NEPA, and RCRA. Staff knew least about the regulations of the Noxious Weed Act, Repatriation Act, and Exotic Species Act; however, several staff members indicated that these acts do not apply to this headquarters office. 


\section{CONCLUSIONS}

\subsection{TRAINING NEEDS}

Staff at a headquarters organization perform diverse tasks in functional areas such as preparing documents, implementing guidance, and overseeing general remedial activities. For the most part, these tasks are the same ones that staff should perform and like to perform.

Once relevant tasks were identified, the skills needed to carry out these tasks were determined. Of 165 skills, 17 were identified as necessary to perform the majority of tasks. These 17 skills focus on critical thinking, problem solving, communication, and program management. Staff were moderately proficient in these skills. If staff had indicated low proficiency in the skills needed for relevant tasks, one could simply conclude that training is justified for skills associated with those tasks. However, because staff have a working knowledge of most skills, training needs are not directly suggested. Therefore, an alternative way to look at training needs is proposed. The first step is to examine the most important skills that apply to multiple tasks (called common skills). Once the common skills are identified, those skills that underlie various sets of tasks are examined to determine the most important skills in which staff should be highly proficient and, thus, the skills for which training is appropriate.

Table 11 lists common skills associated with various tasks. The first of the five columns in the table lists the common skills associated with those tasks that most staff perform (refer back to Table 1 to review the tasks to which these skills apply). Training is valuable for skills associated with those tasks that most staff members perform (and should perform) regularly because these skills are not generally taught in formal science, engineering, and environmental curricula or job training. The second column presents the common skills for tasks that additional staff feel they should perform (these 10 tasks are also listed in Table 1). Considering skills associated with these tasks is worthwhile for assessing training needs because considerably more staff are likely to be called on to perform these tasks when time and resources allow.

The next two columns in Table 11 are related. The third column lists the common skills for those tasks performed least well (see Table 4). The fourth column lists the common skills associated with the tasks for which staff indicated wide ranges of ability (see Table 6). Staff need to be sufficiently trained in these skills to consistently perform the relevant tasks well.

The fifth column of Table 11 lists the common skills for the least preferred tasks that staff perform (see Table 8). This category is valuable because it can be argued that one reason certain tasks are less desirable is that staff feel less confident in their abilities to perform them well. 
TABLE 11 Common Skills Associated with Various Sets of Tasks

\begin{tabular}{|c|c|c|c|c|}
\hline $\begin{array}{l}\text { Common Skills for Tasks that Most } \\
\text { Staff Perform }\end{array}$ & $\begin{array}{l}\text { Common Skills for Tasks that More } \\
\text { Staff Should Perform }\end{array}$ & $\begin{array}{l}\text { Common Skills for Tasks } \\
\text { Performed Least Well }\end{array}$ & $\begin{array}{c}\text { Common Skills for Tasks with } \\
\text { Relatively Wide Ranges } \\
\text { of Ability }\end{array}$ & $\begin{array}{l}\text { Common Skills for Least } \\
\text { Preferred Tasks }\end{array}$ \\
\hline $\begin{array}{l}\text { EM's mission, policies, } \\
\text { management plans, procedures, } \\
\text { priorities, goals, and objectives }\end{array}$ & $\begin{array}{l}\text { EM's mission, policies, } \\
\text { management plans, procedures, } \\
\text { priorities, goals, and objectives }\end{array}$ & $\begin{array}{l}\text { EM's mission, policies, } \\
\text { management plans, procedures, } \\
\text { priorities, goals, and objectives }\end{array}$ & $\begin{array}{l}\text { EM's mission, policies, } \\
\text { management plans, procedures, } \\
\text { priorities, goals, and objectives }\end{array}$ & $\begin{array}{l}\text { EM's mission, policies, } \\
\text { management plans, procedures, } \\
\text { priorities, goals, and objectives }\end{array}$ \\
\hline $\begin{array}{l}\text { Keeping abreast of current } \\
\text { developments }\end{array}$ & $\begin{array}{l}\text { Keeping abreast of current } \\
\text { developments }\end{array}$ & $\begin{array}{l}\text { Keeping abreast of current } \\
\text { developments }\end{array}$ & $\begin{array}{l}\text { Keeping abreast of current } \\
\text { developments }\end{array}$ & $\begin{array}{l}\text { Keeping abreast of current } \\
\text { developments }\end{array}$ \\
\hline Defining applicable criteria & Defining applicable criteria & Defining applicable criteria & Defining applicable criteria & \\
\hline \multirow[t]{2}{*}{$\begin{array}{l}\text { Providing clear.guidance and } \\
\text { direction to accomplish tasks }\end{array}$} & $\begin{array}{l}\text { Providing clear guidance and } \\
\text { direction to accomplish tasks }\end{array}$ & $\begin{array}{l}\text { Providing clear guidance and } \\
\text { dircction to accomplish tasks }\end{array}$ & $\begin{array}{l}\text { Providing clear guidance and } \\
\text { direction to accomplish tasks }\end{array}$ & \\
\hline & $\begin{array}{l}\text { Identifying relevant } \\
\text { material/requirements }\end{array}$ & $\begin{array}{l}\text { Identifying relevant } \\
\text { material/requirements }\end{array}$ & $\begin{array}{l}\text { Identifying relevant } \\
\text { material/requirements }\end{array}$ & $\begin{array}{l}\text { Identifying relevant } \\
\text { material/requirements }\end{array}$ \\
\hline Addressing schedule constraints & & Addressing schedule constraints & Addressing schedule constraints & \\
\hline Organizing/categorizing information & & Organizing/categorizing information & & \\
\hline $\begin{array}{l}\text { Assessing strengths and weaknesses } \\
\text { of alternatives }\end{array}$ & & $\begin{array}{l}\text { Assessing strengths and weaknesses } \\
\text { of altematives }\end{array}$ & & \\
\hline Writing clearly and succinctly & & & & Writing clearly and succinctly \\
\hline $\begin{array}{l}\text { Examining trade-offs among } \\
\text { altematives }\end{array}$ & & $\begin{array}{l}\text { Examining trade-offs among } \\
\text { altematives }\end{array}$ & & \\
\hline DOE management strategies & & & DOE management strategies & \\
\hline & & $\begin{array}{l}\text { Project or program management } \\
\text { techniques }\end{array}$ & $\begin{array}{l}\text { Project or program management } \\
\text { techniques }\end{array}$ & \\
\hline
\end{tabular}




\section{TABLE 11 (Cont.)}

\begin{tabular}{|c|c|c|c|c|}
\hline $\begin{array}{l}\text { Common Skills for Tasks that Most } \\
\text { Staff Perform }\end{array}$ & $\begin{array}{c}\text { Common Skills for Tasks that More } \\
\text { Staff Should Perform }\end{array}$ & $\begin{array}{l}\text { Common Skills for Tasks } \\
\text { Performed Least Well }\end{array}$ & $\begin{array}{l}\text { Common Skills for Tasks with } \\
\text { Relatively Wide Ranges } \\
\text { of Ability }\end{array}$ & $\begin{array}{l}\text { Common Skills for Least } \\
\text { Preferred Tasks }\end{array}$ \\
\hline \multicolumn{5}{|l|}{ Prioritizing based on criteria } \\
\hline \multicolumn{5}{|l|}{$\begin{array}{l}\text { Organizing information and } \\
\text { presenting cogent arguments }\end{array}$} \\
\hline & & $\begin{array}{l}\text { Interagency agreements, } \\
\text { administration, and process }\end{array}$ & & \\
\hline & & Analyzing information & & \\
\hline \multicolumn{5}{|c|}{$\begin{array}{l}\text { Planning, programming, funding, } \\
\text { and acquisition management }\end{array}$} \\
\hline
\end{tabular}


More training in the common skills underlying these tasks could further improve staff proficiency in the relevant skills and make the tasks more desirable.

Training is appropriate for all 17 common skills presented in Table 11; these skills focus on critical thinking/problem solving, communications, and program management. However, it may be impossible to train for these 17 skills simultaneously. Thus, each skill has been further prioritized to assist in implementing training activities (Table 12).

Three criteria were used to determine priorities for training needs: (1) the number of task sets to which the skill applied, (2) the mean competency level for the skill, and (3) the standard deviation for the mean competency of the skill. If these criteria were considered separately, the priority would be as follows:

- Skills that apply to many sets of tasks would receive a higher priority than those that apply to fewer sets of tasks;

- Skills for which staff have low mean competency scores would receive higher priority than those with high mean competency scores; and

- Skills for which staff collectively have a wide variation in competency would be of higher priority than those with a small variation.

To be as comprehensive as possible, these three criteria were considered together. For example, a skill that applied to multiple sets of tasks, had a low mean competency level, and had a large standard deviation would suggest the highest priority for training. On the other hand, a skill that applied to only two sets of tasks and had a high mean competency score would be considered of lower priority for training.

This scheme suggests three levels of priority for staff to further improve their proficiency in the 17 common skills. Four skills were assigned first priority for training:

- Keeping abreast of current developments;

- Defining applicable criteria;

- Understanding of "EM's mission, policies, management plans, procedures, priorities, goals, and objectives"; and

- Understanding of "planning, programming, funding, and acquisition management." 
TABLE 12 Priority Ranking for 17 Common Skills for which Training Is Most Important

\begin{tabular}{|c|c|c|c|c|}
\hline Skill & $\begin{array}{l}\text { Number of } \\
\text { Task } \\
\text { Groups to } \\
\text { which Skill } \\
\text { Applied }^{\mathrm{a}}\end{array}$ & $\begin{array}{l}\text { Mean } \\
\text { Proficiency } \\
\text { Level }^{\mathrm{b}}\end{array}$ & $\begin{array}{l}\text { Standard } \\
\text { Deviation }^{\mathrm{c}}\end{array}$ & Priority \\
\hline \multicolumn{5}{|l|}{ Communications } \\
\hline $\begin{array}{l}\text { Providing clear guidance and direction to } \\
\text { accomplish tasks }\end{array}$ & 4 & 3.0 & 0.7 & 2 \\
\hline Writing clearly and succinctly & 2 & 3.0 & 0.7 & 3 \\
\hline $\begin{array}{l}\text { Interagency agreements, administration, } \\
\text { and process }\end{array}$ & 1 & 2.7 & 0.7 & 2 \\
\hline \multicolumn{5}{|l|}{ Problem solving/critical thinking } \\
\hline Identifying relevant material/requirements & 4 & 2.8 & 0.7 & 2 \\
\hline Organizing/categorizing information & 2 & 3.1 & 0.8 & 2 \\
\hline Keeping abreast of current developments & 5 & 3.0 & 0.8 & 1 \\
\hline Defining applicable criteria & 4 & 2.8 & 0.7 & 1 \\
\hline Analyzing information & 1 & 3.1 & 0.5 & 3 \\
\hline $\begin{array}{l}\text { Organizing information and presenting } \\
\text { cogent arguments }\end{array}$ & 1 & 3.1 & 0.7 & 3 \\
\hline Examining trade-offs among alternatives & 2 & 2.9 & 0.7 & 3 \\
\hline $\begin{array}{l}\text { Assessing strengths and weaknesses of } \\
\text { alternatives }\end{array}$ & 2 & 3.1 & 0.7 & 3 \\
\hline Prioritizing based on criteria & 1 & 3.1 & 0.7 & 3 \\
\hline \multicolumn{5}{|l|}{ Program/project management } \\
\hline $\begin{array}{l}\text { EM's mission, policies, management plans, } \\
\text { procedures, priorities, goals, and } \\
\text { objectives }\end{array}$ & 5 & 2.7 & 0.7 & 1 \\
\hline Addressing schedule constraints & 3 & 2.9 & 0.7 & 3 \\
\hline Project or program management techniques & 2 & 2.9 & 0.9 & 2 \\
\hline DOE management strategies & 2 & 2.4 & 0.7 & 2 \\
\hline $\begin{array}{l}\text { Planning, programming, funding, and } \\
\text { acquisition management }\end{array}$ & 1 & 2.5 & 0.9 & 1 \\
\hline
\end{tabular}

a As illustrated in Table 11.

b Mean proficiency level is based on a 4-point scale: $1=$ no knowledge; 2 = familiarity; $3=$ working knowledge; 4 = expert.

c Standard deviation, or amount of variability, of mean proficiency level. 
Six skills were assigned to second priority:

- Providing clear guidance and direction to accomplish tasks;

- Identifying relevant material/requirements;

- Organizing/categorizing information;

- Project or program management techniques;

- DOE management strategies; and

- Interagency agreements, administration, and process.

Seven skills were assigned to third priority:

- Writing clearly and succinctly,

- Analyzing information,

- Organizing information and presenting cogent arguments,

- Examining trade-offs among alternatives,

- Assessing strengths and weaknesses of alternatives,

- Prioritizing based on criteria, and

- Addressing schedule constraints.

\subsection{FUTURE DIRECTIONS}

The results of this project suggest many approaches for continuing to improve the knowledge and skills of staff. Possible next steps are briefly described below.

\subsubsection{Develop Training Activities}

Now that training needs have been identified, staff must be given an opportunity to meet these needs. Training activities should focus on improving communication skills, critical thinking 
techniques, and program management skills. Different versions of the training may be appropriate for specific job categories or tasks performed. And, of course, diverse formats for training may be appropriate. For example, it may be useful to obtain reference materials and tools (e.g., software) so that staff can be involved in self-paced training.

Different types of existing training activities should be identified, and new training programs may need to be developed. Training courses within the government (e.g., DOE core project management courses, budget courses, technical courses) may be available from various offices within EM or DOE, the Office of Personnel Management, or other federal agencies. Training opportunities outside of DOE and the federal government should also be explored. For example, discussions with U.S. corporations could help identify successful training opportunities to improve staff's knowledge and skills and to promote staff performance.

Other types of opportunities for helping staff improve their skills should also be explored. Pairing experienced staff with less experienced staff and using in-house mentors could improve job proficiency and staff interaction.

\subsubsection{Evaluate the Effectiveness of Training Activities}

While it is critical to ensure that staff have opportunities to participate in training and related experiences, it is equally critical that these opportunities be monitored and assessed to determine how well they are working. Only with such feedback can training opportunities be continually enhanced to better help staff develop the appropriate skills. Effective evaluation includes identifying the purposes of the training, developing data collection materials to test whether the purposes have been achieved, determining how participants will be selected, analyzing the results, and using the results to improve the training.

\subsubsection{Demonstrate Management's Commitment}

Management of this headquarters organization should continue to demonstrate its commitment to professional and personal development for its staff by ensuring that the necessary administrative structures and personnel processes are in place so that training can occur in a timely manner. Actions that managers can take to demonstrate their commitment to meeting training needs are varied. In fact, some of these actions are already in place in varying degrees, although they can be further institutionalized.

Managers can develop and see that Individual Development Plans (IDPs) are implemented. IDPs are the roadmaps between two parties to achieve an agreed-upon goal. Supervisors should work closely with their staff to develop IDPs that reflect the training needs identified in this document and 
the professional and personal training needs of each individual. Goals should be set for individual hours of training to be attended. Supervisors and staff should review IDPs regularly (e.g., twice a year) to evaluate the training process.

Moreover, management can empower staff to use knowledge and skills learned through training opportunities. This empowerment might be accomplished by sharing tasks usually performed by management, such as conducting briefings on projects in which staff have been greatly involved and delegating authority to staff members who are capable of performing tasks.

Managers can also provide flexible training schedules that take into account program activities and can ensure that staff attend the scheduled training. In addition, managers can keep lines of communication open by setting up and monitoring a training schedule, distributing training information to all staff, and assisting in the administrative process of requesting training. Management can also distribute feedback from training courses attended throughout the headquarters organization, share upcoming professional opportunities, and follow through on commitments made at retreats and other self-evaluation activities.

\subsubsection{Explore Related Data Applications}

This report focuses on the tasks and associated skills necessary for this headquarters organization to meet its mission. The information collected in this survey can be viewed from other perspectives to suggest alternative mechanisms for professional development. For instance, the findings reveal the staff's least preferred tasks (see Table 8). Within this headquarters organization, the least preferred tasks might be rotated so as to minimize the negative impact. Rotational assignments with operations offices or with other EM offices might also be considered.

Management of this headquarters organization could also provide staff with more opportunities to perform the tasks they view as most desirable (see Table 8). These tasks use skills such as knowledge of remediation techniques and of the RCRA/CERCLA process (see Table 9), which are not common skills for other sets of tasks (see Table 11) and thus may be used less often. However, if staff are to perform at their best, they need opportunities to perform tasks and use skills they find desirable.

\subsubsection{Apply Methodology to Other Offices}

From a broader EM perspective, it will be important to validate and replicate this study with other organizational units at both headquarters and operations offices to ensure that all staff within EM are performing their jobs as effectively as possible. 


\section{REFERENCES}

American Society for Training and Development, 1990, Be a Better Needs Analyst, INFO-LINE, Issue 502, Alexandria, Va.

Birnbrauer, H., and L.A. Tyson, 1985, "The How To's of Needs Analysis," Technical and Skills Training Programs, H. Birnbrauer (editor), American Society for Training and Development, Washington, D.C.

Close, J.E., 1987, unpublished information, New York State Department of Environmental Conservation, Albany, N.Y.

Cook, J., and B.J. Seith, 1992, "Designing an Effective Environmental Training Program," Journal of Environmental Regulation 2(1):53-62.

Fisher, S.A., and G.B. Frank, 1992, "The Most from Your Training Dollars," The National Public Accountant March:18-21.

Green T.B., et al., 1985, "The Practice of Management: Knowledge vs. Skills," Training and Development Journal July:56-58.

Hensley, J., and C. Young, 1995, personal communication from Hensley and Young (Argonne National Laboratory, Argonne, Ill.) to J.C. Lehr (U.S. Department of Energy, Office of Northwestern Area Programs, Germantown, Md.), July 14.

Hubble, L., and R. Green, 1992, "State Training Needs Assessment," The Public Manager, The New Bureaucrat Summer:33-36.

Kleiman, C., 1993, “As Pay Raises Shrink, Company Perks Grow," Chicago Tribune, Chicago, Il., Feb. 14.

Kosecoff, J., and A. Fink, 1982, Evaluation Basics: A Practitioner's Manual, Sage Publications, Newbury Park, Calif.

Marshall, M.E., and D.W. Mayer, 1992, "Environmental Training: It's Good Business," Business Horizons March/April:54-57.

Meshenberg, M.J., et al., 1990, unpublished information, Argonne National Laboratory, Argonne, III. 
Microsoft Corporation, 1992, Excel for Windows, computer software spreadsheet.

Nadler, L., 1982, Designing Training Programs: The Critical Events Model, Addison-Wesley, Reading, Mass.

Posner, M., 1989, Foundations of Cognitive Science, MTT Press, Cambridge, Mass.

Segal, J.W., et al., 1985, Thinking and Learning Skills, Vol. 2: Relating Instruction to Research, Lawrence Erlbaum Associates, Hillsdale, N.J.

Young, C., and J. Hensley, 1995, "Determining Training Needs from Supervisors' Assessment of Staff Proficiency in Tasks and Skills," in proceedings of the WM '95 Conference, HLW, LLW, Mixed Wastes and Environmental Restoration - Working Towards a Cleaner Environment, Feb. 26 to March 2, 1995, WM Symposia, Inc., Tucson, Ariz.

Zemke, R., 1985, "Needs Assessment: A Brief Overview," Technical and Skills Training Programs, H. Birnbrauer (editor), American Society for Training and Development, Washington, D.C. 

A-1

APPENDIX A:

DATA COLLECTION WORKBOOK 
A-2 


\section{INSTRUCTIONS FOR TRAINING ASSESSMENT/PROFESSIONAL DEVELOPMENT WORKBOOK}

This headquarters organization desires to promote opportunities for your training and professional development so you can feel satisfaction from performing your job effectively and thoroughly. ANL has developed the attached workbook to facilitate this goal. The workbook is designed to inventory the types of activities in which you are involved, to solicit the importance of various skills you feel are necessary for accomplishing various tasks, and to describe your present skills. We are also interested in the types of technical knowledge you use and the regulations that you need to understand.

No one is required to participate in this assessment; it is strictly voluntary. The data collected from this workbook will be confidential. The results will be consolidated into job categories and will not be made available to supervisors for performance or qualification purposes. The information will be tabulated and used to make recommendations about needed training for all staff members. Completion of this workbook is your opportunity to provide input about the types of skills you feel you and other staff need, activities that are not central to your job, and the types of training that interest you.

The workbook is divided into three parts:

Part 1 - Tasks

Part 2 - Ideal Skills for Each Task

\section{Part 3 - Skills}

\section{PART 1 - TASKS}

Part 1 lists, down the left side, the types of activities or tasks that you might perform. As you complete this table, consider tasks that you perform on a routine basis, as well as those that may be performed by you on an irregular basis. These may be tasks listed in your position description, assigned by your supervisor or based on DOE policy.

Please provide your name and position title on the top right-hand corner. This confidential information will be used to group similar types of job categories for analysis.

Please place a response in each cell of all five columns so that every cell has an answer. For each task listed in the left-hand column, please indicate the following: 


\section{Column 1:}

Circle "Yes" or "No" for whether you perform the particular task. You may not perform any number of these tasks for any number of reasons (e.g., "not in my job description," don't feel as you need to, don't have the time, or don't know how), so don't be concerned about answering "No." At the end of Part 1, please list additional tasks you currently perform that are not listed (both routine and irregular).

\section{Column 2:}

Circle the number that best indicates how much you like doing the particular task. Circle "NA" if you do not perform the particular task as indicated in Column 1. Please fill in all cells using the following scale:

$$
\begin{aligned}
1 & =\text { not at all } \\
2 & =\text { a little } \\
3 & =\text { somewhat } \\
4 & =\text { a great deal } \\
\mathrm{NA} & =\text { not applicable }
\end{aligned}
$$

\section{Column 3:}

For those tasks to which you answered "Yes" in Column 1, circle the number that best indicates the degree to which you feel you are currently prepared to perform the task. Circle "NA" if you do not perform the particular task as indicated in Column 1. Please fill in all cells using the following scale:

$$
\begin{aligned}
1 & =\text { not at all } \\
2 & =\text { somewhat well } \\
3 & =\text { sufficiently well } \\
4 & =\text { extremely well } \\
\mathrm{NA} & =\text { not applicable }
\end{aligned}
$$

\section{Column 4:}

Circle "Yes" or "No" for whether you should perform the particular task, but do not for whatever reasons. You should provide a response for each cell. If you answered "Yes" in Column 1 and "No" in Column 4, it will indicate that you perform a particular task that you do not believe you should perform. Likewise, a response of "No" in Column 1 and a "Yes" in Column 4 indicates a task that you currently do not perform, for whatever reason, but you believe you should perform. 


\section{Column 5:}

If you circled "Yes" in Column 4 for a particular task, circle the number that best indicates how much you would like to do this task. If you circled "Yes" in Column 1 and Column 4, your answer in Column 5 should be the same as your answer in Column 2. If you circled "No" in Column 4 for a particular task, circle "NA." Please fill in all cells using the following scale:

$$
\begin{aligned}
1 & =\text { not at all } \\
2 & =\text { a little } \\
3 & =\text { somewhat } \\
4 & =\text { a great deal } \\
\text { NA } & =\text { not applicable }
\end{aligned}
$$

\section{PART 2 - IDEAL SKILLS FOR EACH TASK}

This part of the workbook examines your judgment of the knowledge or skills necessary to perform various tasks even though you may not perform them. Each task will likely require more than one area of knowledge or skill, so each cell of the matrix should have some number circled. These ratings should be based on the knowledge or skills you think well-trained employees need in ideal situations for performing the tasks.

For each individual task listed across the top of the page, please work down the column of cells, circling the number 1 to 4 to indicate how important, in your judgment, each of the 78 areas of knowledge or skills, listed down the left side of the table, is for completing each of the 52 tasks, listed across the top of the table. For example, when executing the task of "PREPARE DOCUMENTS," how important is each area of knowledge or skill (e.g., national consensus standards; interagency agreements, administration, and process; organizing information and present cogent arguments). Please fill in all cells using the following scale:

$1=$ not at all important (irrelevant for the task)

2 = of little importance (some minimum level of knowledge or skill needed to perform task)

3 = important (needed to perform the task well)

4 = of great importance (critical, one cannot perform this task without knowledge or skills in this area) 


\section{PART 3 - SKILLS}

In this third part of the workbook, we want to know about your personal level of expertise. This part of the workbook is divided into the following subsections: general knowledge or skills, technical knowledge, and regulatory knowledge. At the end of Part 3, please list additional knowledge or skills you desire but were not listed.

Please circle the number that specifies your personal level of knowledge or skill. Please fill in all cells using the following scale:

$$
\begin{aligned}
& 1 \text { = little or no knowledge (no understanding) } \\
& 2 \text { = familiarity (some understanding) } \\
& 3 \text { = working knowledge (enough understanding to answer questions asked by others) } \\
& 4 \text { = highly proficient/expert (could teach topic on particular knowledge or skill) }
\end{aligned}
$$

Finally, we would like for you to provide a list of training courses (either by specific title or by subject) that you need to take or would like to take. 


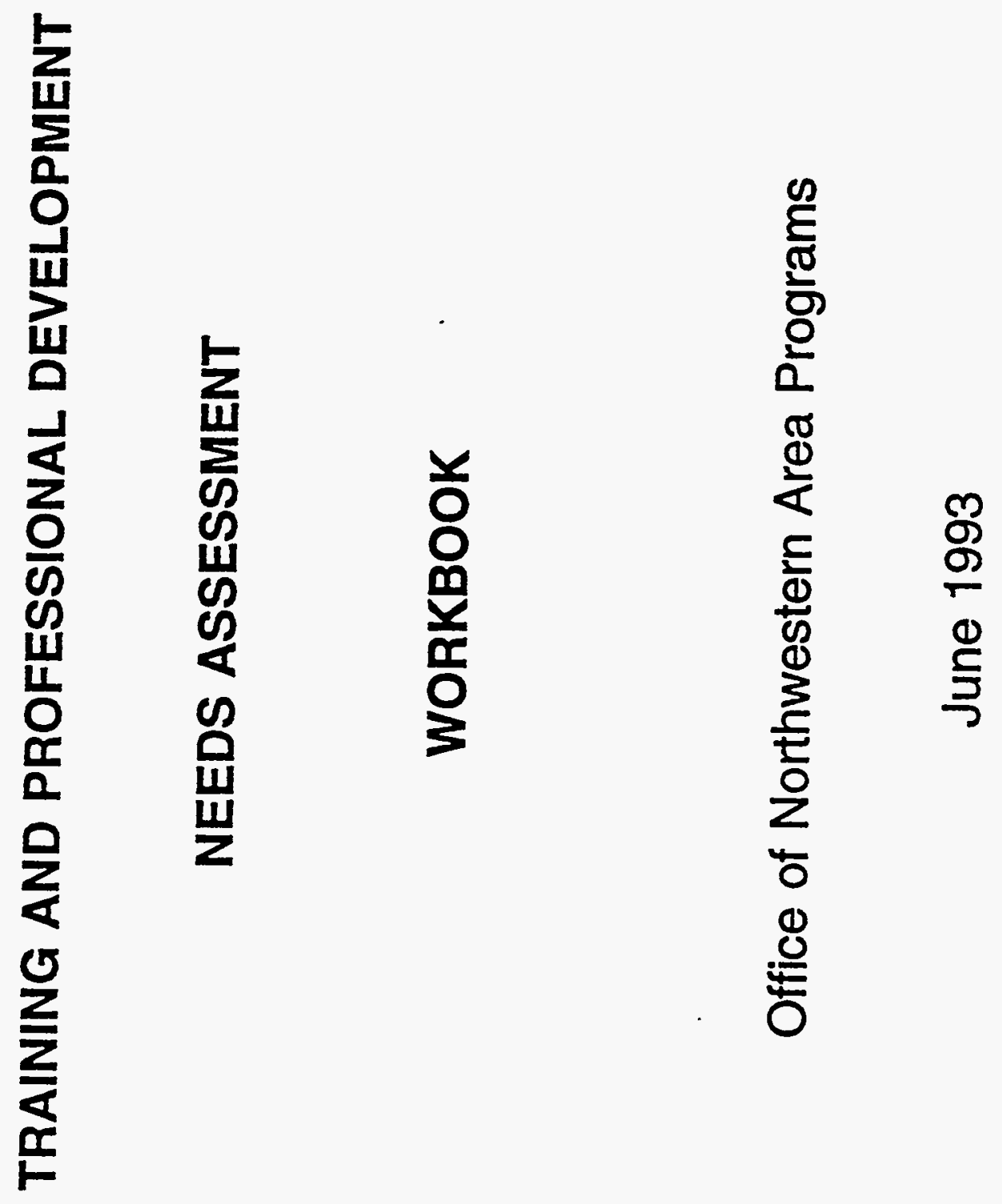


A-8

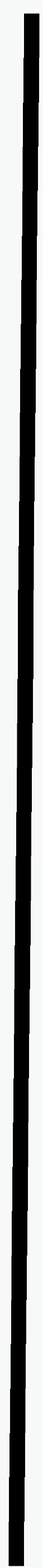


PART 1 - IASKS

Name:

Position Title:

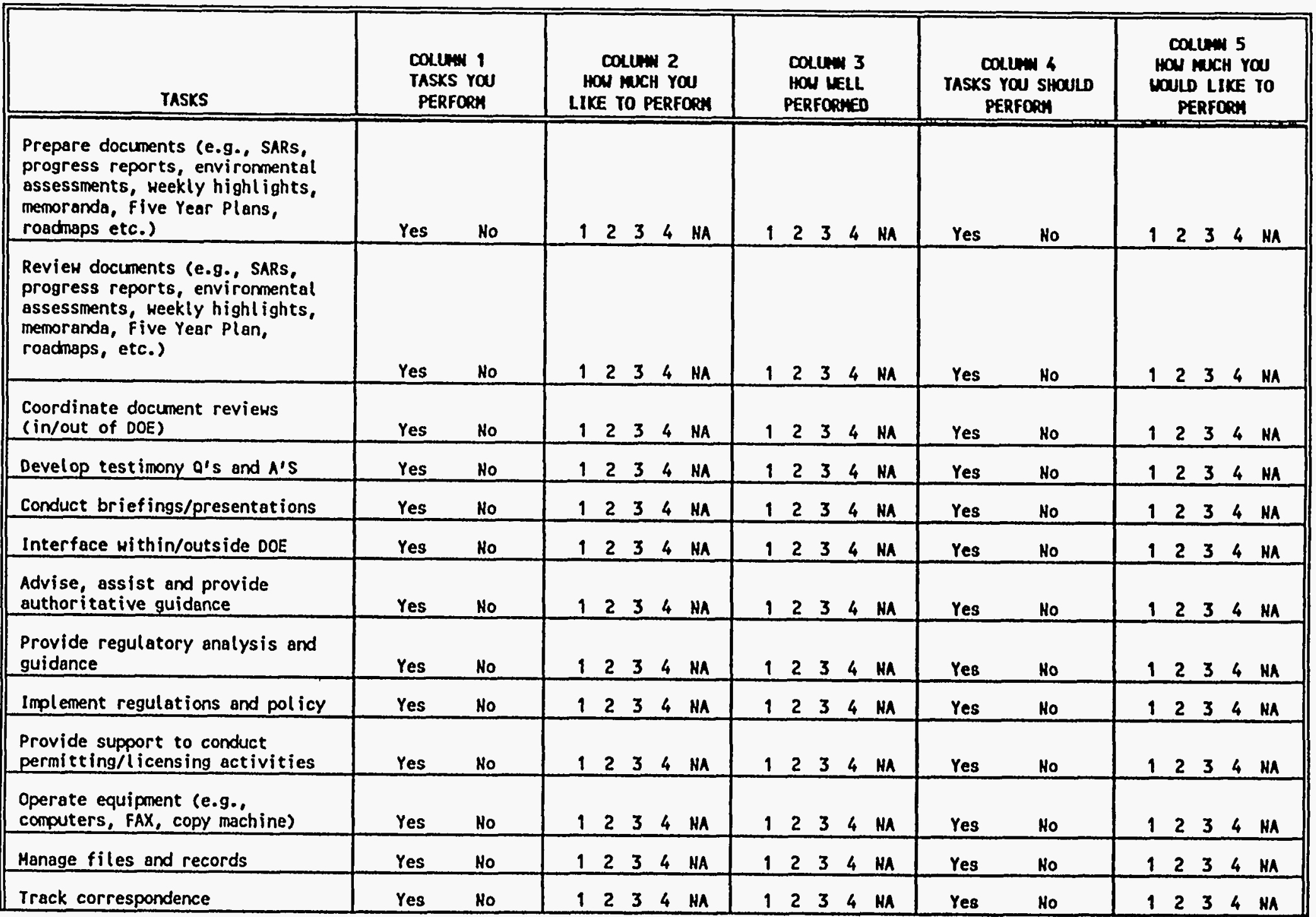


PART 1 - TASKS

\begin{tabular}{|c|c|c|c|c|c|c|c|}
\hline rasKS & $\begin{array}{r}\text { COL } \\
\text { TAS } \\
\text { PE } \\
\end{array}$ & & $\begin{array}{l}\text { COLUNM } 2 \\
\text { HON MUCH YOS } \\
\text { LIKE TO PERFORM } \\
\end{array}$ & $\begin{array}{l}\text { COLUNW } 3 \\
\text { HON MELL } \\
\text { PERFOREDD }\end{array}$ & TASXs & $\begin{array}{l}4 \\
\text { SHOULD } \\
\end{array}$ & $\begin{array}{l}\text { COLUW } 5 \\
\text { HOU MUCH YOS } \\
\text { WOULD LIKE TO } \\
\text { PERFORA }\end{array}$ \\
\hline $\begin{array}{l}\text { Perform travel administrative } \\
\text { functions }\end{array}$ & Yes & Ho & $1234 \mathrm{HA}$ & $1234 \mathrm{HA}$ & Yes & Ho & $1234 \mathrm{HA}$ \\
\hline $\begin{array}{l}\text { Participate in personnel training } \\
\text { and development }\end{array}$ & Yes & No & $1234 \mathrm{NA}$ & $1234 \mathrm{MA}$ & Yes & Ho & $1234 \mathrm{HA}$ \\
\hline $\begin{array}{l}\text { Oversee data/information } \\
\text { management (e.g., ORPS) }\end{array}$ & Yes & No & $1234 \mathrm{HA}$ & $\begin{array}{lllll}1 & 2 & 3 & 4 \mathrm{HA}\end{array}$ & Yes & No & $1234 \mathrm{HA}$ \\
\hline Address personnel/staffing issues & Yes & No & $\begin{array}{lllll}1 & 2 & 3 & 4 & \mathrm{NA} \\
\end{array}$ & $\begin{array}{llll}1 & 2 & 3 & 4 \\
\mathrm{MA}\end{array}$ & Yes & No & $\begin{array}{llll}1 & 234 \mathrm{HA}\end{array}$ \\
\hline $\begin{array}{l}\text { Track and verify corrective } \\
\text { actions }\end{array}$ & Yes & No & $1234 \mathrm{NA}$ & $\begin{array}{llll}1 & 2 & 3 & \mathrm{HA}\end{array}$ & Yes & No & $1234 \mathrm{HA}$ \\
\hline $\begin{array}{l}\text { Oversee surveillance/ } \\
\text { monitoring/inspection activities }\end{array}$ & Yes & No & $\begin{array}{lllll}1 & 2 & 3 & 4\end{array}$ & $1234 \mathrm{MA}$ & Yes & Ho & $\begin{array}{llll}1 & 2 & 3 & 4\end{array}$ \\
\hline $\begin{array}{l}\text { Investigate complaints, } \\
\text { allegations, and incidents }\end{array}$ & Yes & No & $1234 \mathrm{NA}$ & $123 \quad 34 \mathrm{MA}$ & Yes & No & $1234 \mathrm{HA}$ \\
\hline $\begin{array}{l}\text { Provide analyses to determine } \\
\text { effects of remediation projects }\end{array}$ & Yes & No & $1234 \mathrm{HA}$ & $\begin{array}{lllll}1 & 2 & 3 & 4 & \mathrm{MA} \\
\end{array}$ & Yes & No & $\begin{array}{llll}1 & 2 & 3 & 4\end{array}$ \\
\hline $\begin{array}{l}\text { Revien remediation projects and } \\
\text { their progress }\end{array}$ & Yes & No & $1234 \mathrm{NA}$ & $1234 \mathrm{MA}$ & Yes & No & $\begin{array}{llll}1 & 2 & 3 & 4\end{array}$ \\
\hline $\begin{array}{l}\text { Recomend actions to correct } \\
\text { remedial problems }\end{array}$ & Yes & No & $1234 \mathrm{MA}$ & $12334 \mathrm{MA}$ & Yes & No & $1234 \mathrm{HA}$ \\
\hline $\begin{array}{l}\text { Identify and document } \\
\text { deficiencies }\end{array}$ & Yes & No & $1234 \mathrm{MA}$ & $\begin{array}{lllll}1 & 2 & 3 & 4 & \mathrm{MA} \\
\end{array}$ & Yes & Ho & $\begin{array}{lllll}1 & 2 & 3 & 4 & \mathrm{HA}\end{array}$ \\
\hline Set schedules & Yes & No & $1234 \mathrm{NA}$ & $1234 \mathrm{MA}$ & Yes & No & $1234 \mathrm{MA}$ \\
\hline $\begin{array}{l}\text { Identify and conduct facility } \\
\text { acceptance }\end{array}$ & Yes & No & $\begin{array}{llll}1 & 2 & 3 & 4\end{array}$ & $1234 \mathrm{HA}$ & Yes & Ho & $1234 \mathrm{HA}$ \\
\hline $\begin{array}{l}\text { Oversee assessments for clean up } \\
\text { (e.g., risk, feasibility, water } \\
\text { quality, etc.) }\end{array}$ & Yes & No & $1234 \mathrm{NA}$ & $1234 \mathrm{ma}$ & Yes & No & $1234 \mathrm{MA}$ \\
\hline
\end{tabular}


PART 1 - TASKS

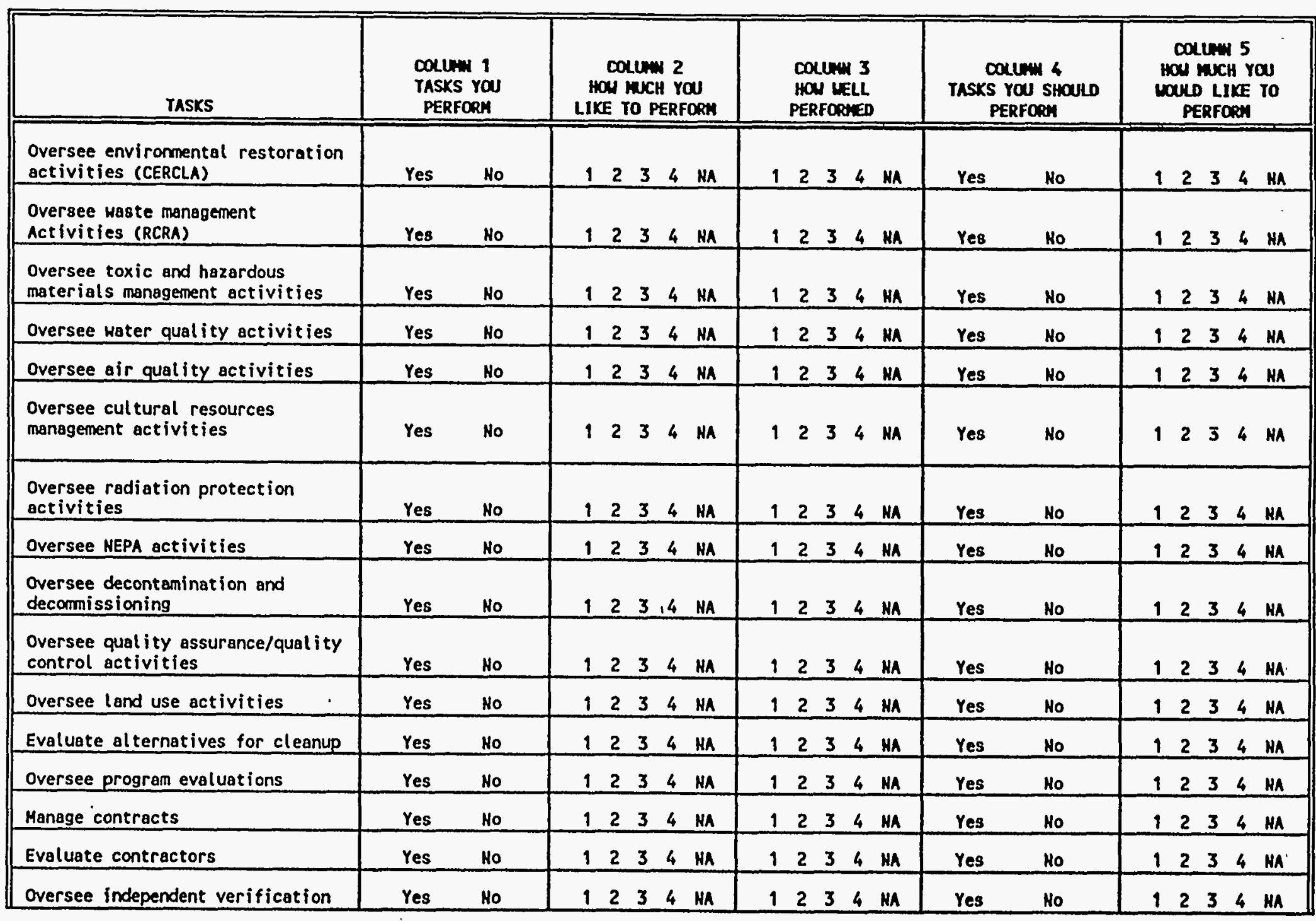


PART 1 - TASKS

\begin{tabular}{|c|c|c|c|c|c|c|c|}
\hline TASKS & & & $\begin{array}{c}\text { COLLWN } 2 \\
\text { HON MUCH YOU } \\
\text { LIKE TO PERFORK }\end{array}$ & $\begin{array}{l}\text { COLUWW } 3 \\
\text { HOU VELL } \\
\text { PERFORMED }\end{array}$ & TASKS & $\begin{array}{l}16 \\
\text { SHOLCD } \\
\text { PAN }\end{array}$ & $\begin{array}{l}\text { COLIUN } 5 \\
\text { HOW MUCH YOU } \\
\text { WOHD LIKE TO } \\
\text { PERFORH }\end{array}$ \\
\hline Identify and allocate resources & Yes & No & $1234 \mathrm{KA}$ & 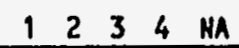 & Yes & Ho & $1234 \mathrm{NA}$ \\
\hline Procure goods and services & Yes & No & $123 \quad 4 \quad \mathrm{HA}$ & $1234 \mathrm{NA}$ & Yes & Ho & $123 \quad 4 \quad \mathrm{NA}$ \\
\hline Oversee cost reviews & Yes & No & $1234 \mathrm{HA}$ & $1234 \mathrm{MA}$ & Yes & Ho & $1234 \mathrm{NA}$ \\
\hline $\begin{array}{l}\text { Formulate, defend, and modify } \\
\text { budget }\end{array}$ & Yes & Ho & $\begin{array}{lllll}1 & 2 & 3 & 4 & \mathrm{HA} \\
\end{array}$ & $1234 \mathrm{HA}$ & Yes & Ho & $1234 \mathrm{HA}$ \\
\hline $\begin{array}{l}\text { Develop, coordinate, and } \\
\text { disseminate program management } \\
\text { policies and procedures }\end{array}$ & Yes & Ho & $1234 \mathrm{HA}$ & 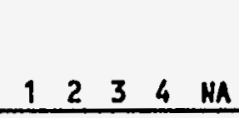 & Yes & Ho & $1234 \mathrm{HA}$ \\
\hline $\begin{array}{l}\text { Establish cost, schedule and } \\
\text { technical baselines }\end{array}$ & Yes & No & $123 \quad 3 \quad \mathrm{NA}$ & $123 \quad 3 \quad \mathrm{HA}$ & Yes & Ho & $1234 \mathrm{HA}$ \\
\hline $\begin{array}{l}\text { Develop resource requirements } \\
\text { (budget/FTEs) }\end{array}$ & Yes & Ho & $\begin{array}{lllll}1 & 2 & 3 & 4 & \mathrm{NA} \\
\end{array}$ & 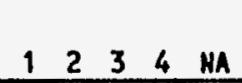 & Yes & Ho & $1234 \mathrm{MA}$ \\
\hline Develop new/improved technologies & Yes & No & $1234 \quad \mathrm{MA}$ & $1234 \mathrm{MA}$ & Yes & Ho & $1234 \mathrm{MA}$ \\
\hline Oversee RODT\&E activities & Yes & No & $1234 \mathrm{HA}$ & $\begin{array}{lllll}1 & 2 & 3 & 4 \mathrm{HA}\end{array}$ & Yes & Ho & $1234 \mathrm{NA}$ \\
\hline other (specify) & Yes & No & $123,4 \mathrm{MA}$ & $\begin{array}{lllll}1 & 2 & 34 \mathrm{HA} \\
\end{array}$ & Yes & Ho & $1234 \mathrm{HA}$ \\
\hline other (specify) & Yes & No & $1234 \mathrm{HA}$ & $\begin{array}{lllll}1 & 2 & 3 & 4 & \mathrm{HA}\end{array}$ & Yes & No & $1234 M A$ \\
\hline Other (specify) & Yes & No & $1234 \mathrm{MA}$ & $\begin{array}{lllll}1 & 2 & 3 & 4 \mathrm{HA}\end{array}$ & Yes & No & $1234 \mathrm{HA}$ \\
\hline Other (specify) & Yes & No & $\begin{array}{lllll}1 & 2 & 3 & 4 & \mathrm{MA} \\
\end{array}$ & $\begin{array}{lllll}1 & 2 & 3 & \mathrm{NA}\end{array}$ & Yes & No & $12334 \mathrm{HA}$ \\
\hline Other (specify) & Yes & No & $1234 \mathrm{MA}$ & $\begin{array}{lllll}1 & 2 & 3 & 4\end{array}$ & Yes & Ho & $12334 \mathrm{NA}$ \\
\hline Other (specify) & Yes & No & $1234 \mathrm{MA}$ & 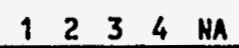 & Yes & Ho & 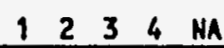 \\
\hline Other (specify) & Yes & Ho & $1234 \mathrm{MA}$ & $1234 \mathrm{MA}$ & Yes & Ho & 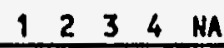 \\
\hline other (specify) & Yes & No & $1234 \mathrm{HA}$ & $1234 \mathrm{NA}$ & Yes & Ho & 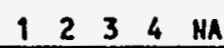 \\
\hline other (specify) & Yes & Ho & $\begin{array}{lllll}1 & 2 & 3 & 4 & \mathrm{HA}\end{array}$ & $1234 \mathrm{NA}$ & Yes & Ho & $1234 \mathrm{MA}$ \\
\hline
\end{tabular}


A- 15

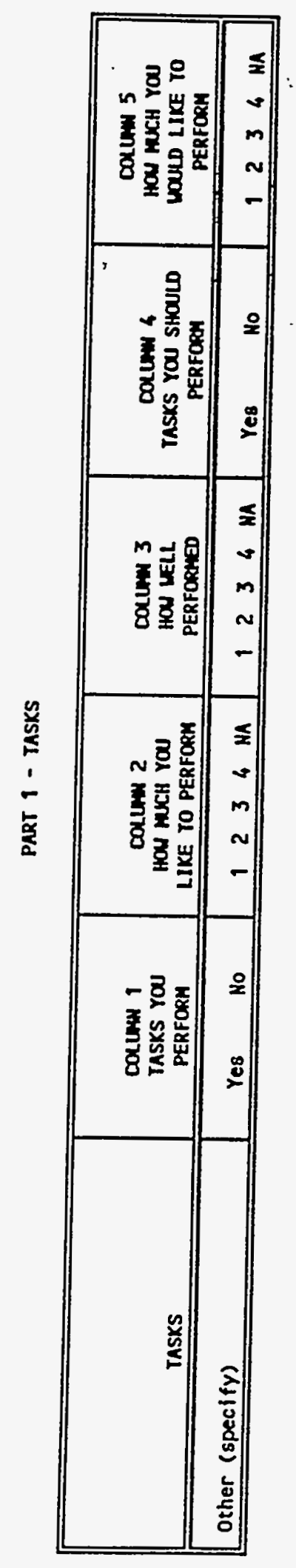

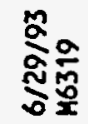


A-16 


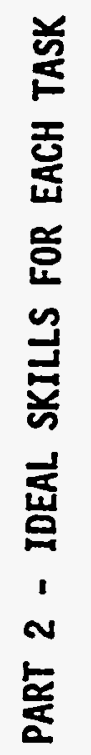


A-18

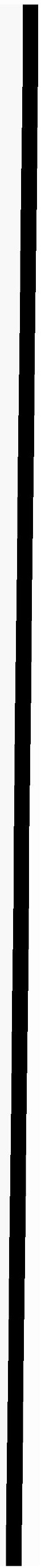




\section{PART 2 - IDEAL SKILLS FOR EACH TASK}

\begin{tabular}{|c|c|c|c|c|c|}
\hline$\frac{\text { TASKS } \rightarrow->}{\text { SXILLS/KMOULEDCE }}$ & 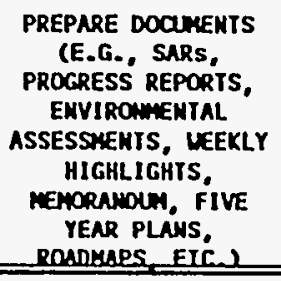 & 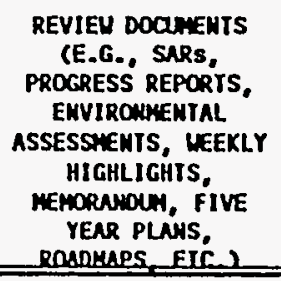 & $\begin{array}{c}\text { COORDIMATE } \\
\text { DOQWEEHS REVIEUS } \\
\text { (IN/OUT OF DOE) } \\
\end{array}$ & $\begin{array}{l}\text { DEVELOP IESTIIOWY } \\
\text { Q'S ANO A'S } \\
\end{array}$ & $\begin{array}{c}\text { COMOUCT } \\
\text { BRIEFIMGS/PRESER- } \\
\text { TATIONS } \\
\end{array}$ \\
\hline Hational consensus standurds & 1234 & 1234 & 1234 & 1234 & 1234 \\
\hline $\begin{array}{l}\text { Interagency agreements, } \\
\text { adninistration, and process }\end{array}$ & 1234 & 1234 & 1234 & 1234 & 1234 \\
\hline $\begin{array}{l}\text { Organizing information and } \\
\text { present cogent arguments }\end{array}$ & 1234 & 1234 & 1234 & 1234 & 1234 \\
\hline Writing succinctly and ctearty & 1234 & 1234 & 1234 & 1234 & 1234 \\
\hline $\begin{array}{l}\text { Public speaking (e.9., public } \\
\text { presentations, employee/employer } \\
\text { interactions) }\end{array}$ & 1234 & 1234 & 1234 & 1234 & 1234 \\
\hline $\begin{array}{l}\text { Interpersonal facility (e.g., } \\
\text { public, media, other employees } \\
\text { and special interest groups) }\end{array}$ & 1234 & 1234 & 1234 & 1234 & 1234 \\
\hline Negotiating/conflict resolution & 1234 & 1234 & 1234 & 1234 & 1234 \\
\hline $\begin{array}{l}\text { Providing clear guidance and } \\
\text { direction to accompl ish tasks }\end{array}$ & $123 \quad 3 \quad 4$ & 1234 & 1234 & 1234 & 1234 \\
\hline Meeting facilitation/leadership & $123 \quad 4$ & 1234 & 1234 & 1234 & 1234 \\
\hline DOE management strategies & 1234 & 1234 & 1234 & 1234 & 1234 \\
\hline $\begin{array}{l}\text { EM's mission, policies, } \\
\text { management plans, procedures, } \\
\text { priorities, gools and objectives }\end{array}$ & 1234 & 1234 & $123 \quad 3 \quad 4$ & 1234 & 1234 \\
\hline DOE departmental organization & 1234 & 1234 & 1234 & 1234 & 1234 \\
\hline $\begin{array}{l}\text { Contractor organization and } \\
\text { procedures }\end{array}$ & 1234 & $123 \quad 3 \quad 4$ & 1234 & 1234 & 1234 \\
\hline Decision analysis & 1234 & 1234 & 1234 & 1234 & 1234 \\
\hline Conducting library research & 1234 & $123 \quad 3$ & 1234 & 1234 & 1234 \\
\hline $\begin{array}{l}\text { Facility, site, operations and } \\
\text { processes }\end{array}$ & 1234 & 1234 & 1234 & 1234 & 1234 \\
\hline Defining boundaries of a problem & 1234 & 1234 & 1234 & 1234 & $\begin{array}{llll}1 & 2 & 3 & 4\end{array}$ \\
\hline
\end{tabular}


PART 2 - IDEAL SKILLS FOR EACH TASK

\begin{tabular}{|c|c|c|c|c|c|}
\hline SKILLS/KMOMLEOCE & 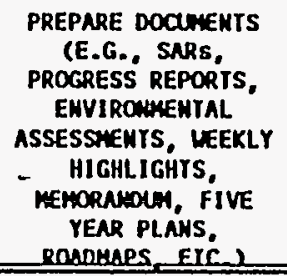 & 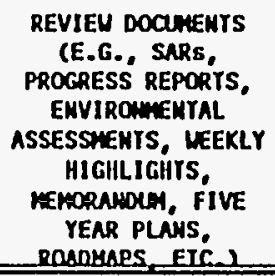 & $\begin{array}{c}\text { COORDIMATE } \\
\text { DOCANENTS REVIEUS } \\
\text { (IH/OUI OF DOE) } \\
\end{array}$ & $\begin{array}{c}\text { DEVELOP TESTIMONY } \\
\text { O'S AND A'S } \\
\end{array}$ & $\begin{array}{c}\text { DOWOUCT } \\
\text { QRIEFINGS/PRESEM- } \\
\text { TATIOWS } \\
\end{array}$ \\
\hline $\begin{array}{l}\text { Keeping abreast of current } \\
\text { developments }\end{array}$ & 1234 & $123 \quad 3$ & 1234 & 1234 & 1234 \\
\hline Organizing/categorizing & 1234 & 1234 & 1234 & 1234 & 1234 \\
\hline $\begin{array}{l}\text { Operating equipment (e.g... } \\
\text { testing equipment, respirators) }\end{array}$ & 1234 & 1234 & 1234 & 1234 & 1234 \\
\hline Operoting and using computer & 1234 & 1234 & 1234 & 1234 & 1234 \\
\hline Touch typing & $123 \quad 4$ & 1234 & 1234 & 1234 & 1234 \\
\hline $\begin{array}{l}\text { Understanding principles of data } \\
\text { base requirements and basel ine } \\
\text { techniques }\end{array}$ & 1234 & 1234 & $123 \quad 34$ & 1234 & 1234 \\
\hline $\begin{array}{l}\text { Using sof tware and applying it } \\
\text { to tasks }\end{array}$ & 1234 & 1234 & 1234 & 1234 & 1234 \\
\hline Time management & 1234 & 1234 & 1234 & 1234 & 1234 \\
\hline Neighing costs versus benefits & 1234 & 1234 & 1234 & 1234 & 1234 \\
\hline $\begin{array}{l}\text { Project or program manogement } \\
\text { techniques }\end{array}$ & 1234 & $\begin{array}{lll} & & 1 \\
123 & 4 \\
\end{array}$ & 1234 & 1234 & 1234 \\
\hline $\begin{array}{l}\text { Principles of DOE budget } \\
\text { formulations, budget execution } \\
\text { and cost accounting, and the } \\
\text { procedures involved }\end{array}$ & 1234 & 1234 & 1234 & 1234 & 1234 \\
\hline Schedule constroints & 1234 & 1234 & 1234 & 1234 & 1234 \\
\hline $\begin{array}{l}\text { Planning, programing, funding } \\
\text { and acquisition manogement }\end{array}$ & 1234 & 1234 & 1234 & 1234 & 1234 \\
\hline $\begin{array}{l}\text { Conducting onalyses involving } \\
\text { complex variables }\end{array}$ & 1234 & 1234 & 1234 & $123 \quad 4$ & 1234 \\
\hline Supervising staff/contractors & 1234 & 1234 & 1234 & 1234 & 1234 \\
\hline $\begin{array}{l}\text { Delegating authority and } \\
\text { responsibility }\end{array}$ & 1234 & $\begin{array}{llll}1 & 2 & 3 & 4\end{array}$ & 1234 & 1234 & 1234 \\
\hline
\end{tabular}


PART 2 - IDEAL SKILLS FOR EACH TASK

\begin{tabular}{|c|c|c|c|c|c|}
\hline$\frac{\text { IASKS } \cdots>}{\text { SXILLS/KWOAREOGE }}$ & 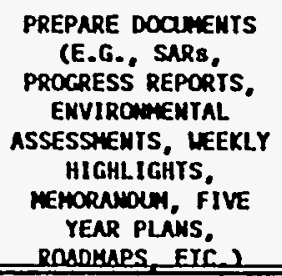 & 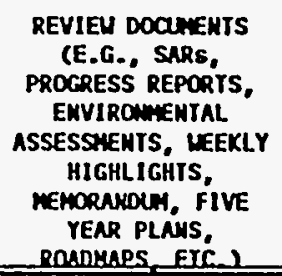 & $\begin{array}{l}\text { COORDIMAIE } \\
\text { DOCOMENTS REVIEUS } \\
\text { (IN/OUT OF DOE) }\end{array}$ & $\begin{array}{l}\text { DEVELOP IESTIMONY } \\
\text { Q'S NDD A'S }\end{array}$ & $\begin{array}{c}\text { CONOUCT } \\
\text { BRIEFINGS/PRESEM- } \\
\text { TAIIOWS } \\
\end{array}$ \\
\hline Equal Opportunity program plans & 1234 & 1234 & 1234 & 1234 & 1234 \\
\hline $\begin{array}{l}\text { Analyzing/synthesizing } \\
\text { information }\end{array}$ & 1234 & 1234 & 1234 & 1234 & 1234 \\
\hline $\begin{array}{l}\text { Assessing strengths and } \\
\text { weaknesses of alternatives }\end{array}$ & 1234 & 1234 & 1234 & 1234 & 1234 \\
\hline $\begin{array}{l}\text { Anticipating unintended } \\
\text { consequences }\end{array}$ & 1234 & 1234 & 1234 & 1234 & 1234 \\
\hline $\begin{array}{l}\text { Comparing existing conditions to } \\
\text { pre-set standards }\end{array}$ & 1234 & 1234 & 1234 & 1234 & 1234 \\
\hline Prioritizing based on criteria & 1234 & 1234 & 1234 & 1234 & 1234 \\
\hline $\begin{array}{l}\text { Identifying relevant } \\
\text { materisl/requirements }\end{array}$ & 1234 & 1234 & 1234 & 1234 & 1234 \\
\hline Delegating tasks & 1234 & $123 \quad 4$ & 1234 & 1234 & 1234 \\
\hline Defining applicable criteria & 1234 & 1234 & 1234 & 1234 & 1234 \\
\hline $\begin{array}{l}\text { Reading technical material for a } \\
\text { set purpose }\end{array}$ & $123 \quad 4$ & 1234 & 1234 & 1234 & $123 \quad 34$ \\
\hline Speed reading & 1234 & 1234 & 1234 & 1234 & 1234 \\
\hline Consolidating materiols & 1234 & 1234 & 1234 & 1234 & 1234 \\
\hline $\begin{array}{l}\text { Examining trade-offs among } \\
\text { alternatives }\end{array}$ & 1234 & 1234 & 1234 & 1234 & 1234 \\
\hline $\begin{array}{l}\text { Geomapping, remote sensing, and } \\
\text { characterization techniques }\end{array}$ & 1234 & 1234 & 1234 & 1234 & 1234 \\
\hline $\begin{array}{l}\text { Proper engineering/technical } \\
\text { standards }\end{array}$ & 1234 & 1234 & 1234 & 1234 & 1234 \\
\hline Volid engineering solutions & 1234 & 1234 & 1234 & 1234 & 1234 \\
\hline Remediation techniques & 1234 & 1234 & 1234 & 1234 & 1234 \\
\hline
\end{tabular}


PART 2 - IDEAL SKILLS FOR EACH TASK

\begin{tabular}{|c|c|c|c|c|c|}
\hline SXILLS/KMOALEOCE & 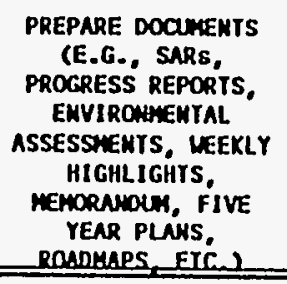 & 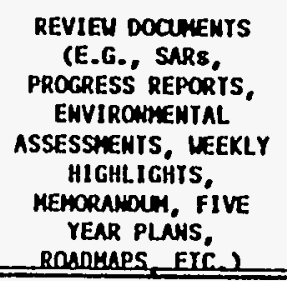 & $\begin{array}{c}\text { COORDIKAIE } \\
\text { DOCWENTS REVIEUS } \\
\text { (IN/OUT OF DOE) } \\
\end{array}$ & $\begin{array}{l}\text { DEVELLP TESTINOWY } \\
\text { O'S NND A'S } \\
\end{array}$ & $\begin{array}{l}\text { COMDUCT } \\
\text { BRIEFINGS/PRESEN- } \\
\text { TATIONS } \\
\end{array}$ \\
\hline $\begin{array}{l}\text { Haste treatment/disposal } \\
\text { technologies }\end{array}$ & 1234 & 1234 & 1234 & $123 \quad 4$ & 1234 \\
\hline Human heal th impocts & 1234 & $123 \quad 4$ & 1234 & 1234 & 1234 \\
\hline Radiation effects & 1234 & 1234 & 1234 & 1234 & 1234 \\
\hline $\begin{array}{l}\text { Heal th and radiological safety } \\
\text { impacts and benefits }\end{array}$ & 1234 & 1234 & 1234 & 1234 & 1234 \\
\hline Heal th physics & 1234 & 1234 & 1234 & 1234 & 1234 \\
\hline Safety and health & 1234 & 1234 & 1234 & 1234 & 1234 \\
\hline Iransportation issues & 1234 & 1234 & 1234 & 1234 & 1234 \\
\hline Packaging issues & 1234 & 1234 & 1234 & 1234 & 1234 \\
\hline Biology, zoology, and ecology & 1234 & 1234 & 1234 & 1234 & 1234 \\
\hline Chemistry & 1234 & 1234 & 1234 & 1234 & 1234 \\
\hline Nucl ear engineering & 1234 & $123^{\prime} 4$ & 1234 & 1234 & 1234 \\
\hline Physical sciences & $123 \quad 3$ & 1234 & 1234 & 1234 & 1234 \\
\hline Geohydrology & 1234 & 1234 & 1234 & 1234 & 1234 \\
\hline Cartography & 1234 & 1234 & 1234 & 1234 & 1234 \\
\hline Geophysics & 1234 & 1234 & 1234 & 1234 & 1234 \\
\hline D8D techniques & 1234 & 1234 & 1234 & 1234 & 1234 \\
\hline Cultural diversity issues & 1234 & 1234 & 1234 & 1234 & 1234 \\
\hline $\begin{array}{l}\text { Hazardous, chemical, radioactive } \\
\text { and mixed weste management }\end{array}$ & 1234 & 1234 & 1234 & 1234 & 1234 \\
\hline RCRA/CERCLA process & 1234 & 1234 & 1234 & 1234 & 1234 \\
\hline Hatural resources & 1234 & 1234 & 1234 & 1234 & 1234 \\
\hline Land use & 1234 & 1234 & 1234 & $\begin{array}{llll}1 & 2 & 3 & 4\end{array}$ & 1234 \\
\hline
\end{tabular}


PART 2 - IDEAL SKILLS FOR EACH TASK

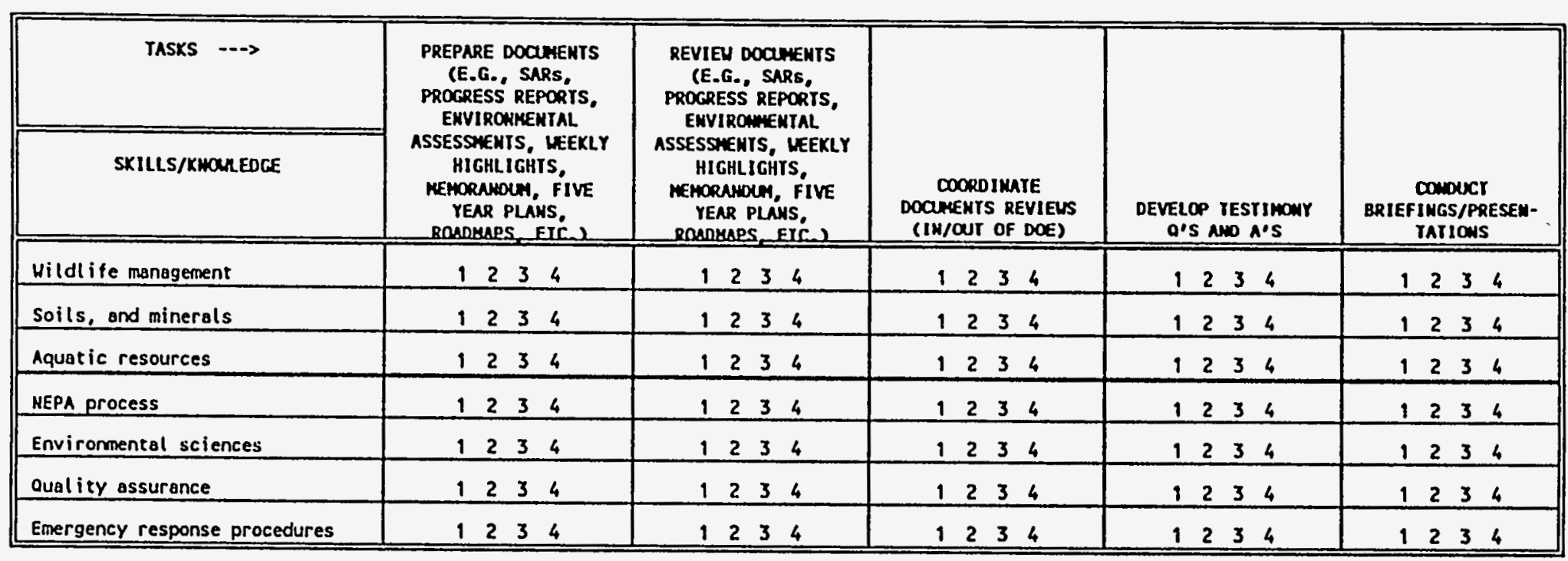


PART 2 - IDEAL SKILLS FOR EACH TASK

\begin{tabular}{|c|c|c|c|c|c|}
\hline rasks $\cdots$ & \multirow[b]{2}{*}{$\begin{array}{c}\text { INTERFACE } \\
\text { WITHIN/OUISIDE DOE }\end{array}$} & \multirow{2}{*}{$\begin{array}{l}\text { ADVISE, ASSIST \& } \\
\text { PROVIDE } \\
\text { MUTHORIYATIVE } \\
\text { RUIDAMCE } \\
\end{array}$} & \multirow[b]{2}{*}{$\begin{array}{l}\text { PROVIDE RECHAATORY } \\
\text { AMALYSIS \& GUIDAHCE }\end{array}$} & \multirow{2}{*}{$\begin{array}{c}\text { PROVIDE SUPPORT TO } \\
\text { COWOUCT } \\
\text { PEPOIIIING/LICENSING } \\
\text { ACLUYUIIES } \\
\end{array}$} & \multirow{2}{*}{$\begin{array}{c}\text { IPPLEKENY } \\
\text { RECAHATIONS \& } \\
\text { POAICY }\end{array}$} \\
\hline SKILLS/KHOAREDCE & & & & & \\
\hline Mational consensus standards & 1234 & 1234 & 1234 & 1234 & 1234 \\
\hline $\begin{array}{l}\text { Interagency agreements, } \\
\text { adninistration, and process }\end{array}$ & 1234 & 1234 & 1234 & 1234 & 1234 \\
\hline $\begin{array}{l}\text { Organizing information and present } \\
\text { cogent arguments }\end{array}$ & 1234 & 1234 & 1234 & 1234 & 1234 \\
\hline Uriting succinctly and clearly & 1234 & 1234 & 1234 & 1234 & 1234 \\
\hline $\begin{array}{l}\text { Publ ic speaking (e.g., public } \\
\text { presentations, employee/employer } \\
\text { interactions) }\end{array}$ & 1234 & 1234 & 1234 & 1234 & 1234 \\
\hline $\begin{array}{l}\text { Interpersonal facility (e.g., } \\
\text { public, media, other employees and } \\
\text { special interest groups) }\end{array}$ & 1234 & 12234 & $123 \quad 3 \quad 4$ & 1234 & 1234 \\
\hline Negotiating/conflict resolution & 1234 & 1234 & 1234 & 1234 & 1234 \\
\hline $\begin{array}{l}\text { Providing clear guidance and } \\
\text { direction to accomplish tasks }\end{array}$ & 1234 & 1234 & 1234 & 1234 & 1234 \\
\hline Meeting facilitation/leadership & 1234 & 1234 & 1234 & 1234 & 1234 \\
\hline DOE management strategies & 1234 & 1234 & 1234 & 1234 & 1234 \\
\hline $\begin{array}{l}\text { EH's mission, policies, management } \\
\text { plans, procedures, priorities, } \\
\text { goals and objectives }\end{array}$ & 1234 & 1234 & 1234 & 1234 & 1234 \\
\hline DOE departmental organization & 1.234 & 1234 & 1234 & 1234 & 1234 \\
\hline $\begin{array}{l}\text { Controctor organization and } \\
\text { procedures }\end{array}$ & 1234 & 1234 & 1234 & 1234 & 1234 \\
\hline Decision analysis & 1234 & 1234 & 1234 & 1234 & 1234 \\
\hline Conduct ing library research & 1234 & 1234 & 1234 & 1234 & 1234 \\
\hline $\begin{array}{l}\text { Facility, site, operations and } \\
\text { processes }\end{array}$ & 1234 & 1234 & 1234 & 1234 & 1234 \\
\hline Defining boundaries of a problem & 1234 & 1234 & 1234 & 1234 & 1234 \\
\hline $\begin{array}{l}\text { Keeping abreast of current } \\
\text { developments }\end{array}$ & 1234 & 1234 & 1234 & 1234 & 1234 \\
\hline
\end{tabular}


PART 2 - IDEAL SKILLS FOR EACH TASK

\begin{tabular}{|c|c|c|c|c|c|}
\hline TASXS $\cdots$ SXILLS/KMOAKEOCE & $\begin{array}{c}\text { INTERFACE } \\
\text { UITHIN/OUISIDE DOE } \\
\end{array}$ & $\begin{array}{c}\text { NDVISE, ASSIST } \\
\text { PROWIDE } \\
\text { MTHORITATIVE } \\
\text { CULDAYCE } \\
\end{array}$ & $\begin{array}{l}\text { PROVIDE RECULATORY } \\
\text { ANALYSIS \& CUIDAKCE } \\
\end{array}$ & $\begin{array}{l}\text { PROVIDE SUPPORT TO } \\
\text { COUDUCT } \\
\text { PERAIITING/LICENSIMG } \\
\text { ACIIUIYIES } \\
\end{array}$ & $\begin{array}{l}\text { IHPLENENT } \\
\text { REGULAIIONS } \\
\text { POLICY } \\
\end{array}$ \\
\hline Organizing/categorizing & 1234 & 1234 & 1234 & 1234 & 1234 \\
\hline $\begin{array}{l}\text { Operating equipment (e.g., testing } \\
\text { equipment, respirators) }\end{array}$ & 1234 & 1234 & 1234 & 1234 & 1234 \\
\hline Operating and using computer & 1234 & 1234 & 1234 & 1234 & 1234 \\
\hline Iouch typing & 1234 & 1234 & 1234 & 1234 & 1234 \\
\hline $\begin{array}{l}\text { Understanding principles of dato } \\
\text { base requirements and basel ine } \\
\text { techniques }\end{array}$ & 1234 & 1234 & 1234 & 1234 & 1234 \\
\hline $\begin{array}{l}\text { Using sof tware and applying it to } \\
\text { tasks }\end{array}$ & 1234 & 1234 & 1234 & 1234 & 1234 \\
\hline rime management & 1234 & 1234 & 1234 & 1234 & 1234 \\
\hline Weighing costs versus benefits & 1234 & 1234 & 1234 & 1234 & 1234 \\
\hline $\begin{array}{l}\text { Project or program management } \\
\text { techniques }\end{array}$ & 1234 & 1234 & 1234 & 1234 & 1234 \\
\hline $\begin{array}{l}\text { Principles of DOE budget } \\
\text { formulations, budget execution and } \\
\text { cost accounting, and the } \\
\text { procedures involved }\end{array}$ & 1234 & 12341 & 1234 & 1234 & 1234 \\
\hline Schedule constraints & 1234 & 1234 & 1234 & 1234 & 1234 \\
\hline $\begin{array}{l}\text { Planning, programing, funding and } \\
\text { acquisition management }\end{array}$ & 1234 & 1234 & 1234 & 1234 & 1234 \\
\hline $\begin{array}{l}\text { Conduct ing analyses involving } \\
\text { complex variables }\end{array}$ & 1234 & 1234 & 1234 & 1234 & 1234 \\
\hline Supervising staff/contractors & 1234 & 1234 & 1234 & 1234 & 1234 \\
\hline $\begin{array}{l}\text { Delegating authority and } \\
\text { responsibility }\end{array}$ & 1234 & 1234 & 1234 & 1234 & 1234 \\
\hline Equal Opportunity program plans & 1234 & 1234 & 1234 & 1234 & 1234 \\
\hline Analyzing/synthesizing information & 1234 & 1234 & 1234 & 1234 & 1234 \\
\hline $\begin{array}{l}\text { Assessing strengths and meaknesses } \\
\text { of al ternatives }\end{array}$ & 1234 & 1234 & 1234 & 1234 & 1234 \\
\hline
\end{tabular}


PART 2 - IDEAL SKILLS FOR EACH TASK

\begin{tabular}{|c|c|c|c|c|c|}
\hline$\frac{\text { TASKS } \cdots>}{\text { SKILLS/KMOMEOCE }}$ & $\begin{array}{c}\text { IHTERFACE } \\
\text { MITHIH/OUISIDE DOE } \\
\end{array}$ & $\begin{array}{c}\text { ADVISE, ASSIST } \\
\text { PROVIDE } \\
\text { AUTHORITATIVE } \\
\text { GULDAYCE } \\
\end{array}$ & $\begin{array}{l}\text { PROVIDE RECUIATORY } \\
\text { AMALYSIS \& GUIDANCE } \\
\end{array}$ & $\begin{array}{l}\text { PROVIOE SUPPORT YO } \\
\text { OONDUCT } \\
\text { PERAITIING/LICENSIKG } \\
\text { ACUIYIYUSS }\end{array}$ & $\begin{array}{c}\text { IMPLEMEMY } \\
\text { RECHLATIONS } \\
\text { POUICY } \\
\end{array}$ \\
\hline $\begin{array}{l}\text { Anticipating unintended } \\
\text { consequences }\end{array}$ & 1234 & 1234 & $123 \quad 4$ & 1234 & 1234 \\
\hline $\begin{array}{l}\text { Conparing existing conditions to } \\
\text { pre-set standards }\end{array}$ & 1234 & 1234 & $123 \quad 4$ & 1234 & 1234 \\
\hline Prioritizing based on criteria & 1234 & 1234 & 1234 & 1234 & 1234 \\
\hline $\begin{array}{l}\text { Identifying relevant } \\
\text { naterial/requirements }\end{array}$ & 1234 & 1234 & 1234 & 1234 & 1234 \\
\hline Delegating tasks & 1234 & 1234 & 1234 & 1234 & 1234 \\
\hline Defining applicable criteria & 1234 & 1234 & 1234 & 1234 & 1234 \\
\hline $\begin{array}{l}\text { Reading technical material for a } \\
\text { set purpose }\end{array}$ & 1234 & 1234 & 1234 & 1234 & 1234 \\
\hline Speed resding & 1234 & 1234 & 1234 & 1234 & 1234 \\
\hline Consol idating materials & 1234 & 1234 & 1234 & 1234 & 1234 \\
\hline $\begin{array}{l}\text { Examining trade-offs among } \\
\text { alternatives }\end{array}$ & 1234 & $\begin{array}{llll}1 & 2 & 3 & 4 \\
\end{array}$ & 1234 & 1234 & 1234 \\
\hline $\begin{array}{l}\text { Geomopping, remote sensing, and } \\
\text { characterization techniques }\end{array}$ & 1234 & 1234 & 1234 & 1234 & 1234 \\
\hline $\begin{array}{l}\text { Proper engineering/technical } \\
\text { standards }\end{array}$ & 1234 & 1234 & 1234 & 1234 & 1234 \\
\hline Valid engineering solutions & 1234 & 1234 & 1234 & 1234 & 1234 \\
\hline Remediat ion techniques & 1234 & 1234 & 1234 & 11234 & 1234 \\
\hline $\begin{array}{l}\text { Waste treatment/disposal } \\
\text { technologies }\end{array}$ & 1234 & 1234 & 1234 & 1234 & 1234 \\
\hline Human health impacts & $\begin{array}{llll}1 & 2 & 3 & 4\end{array}$ & 1234 & 1234 & 1234 & 1234 \\
\hline Radiation effects & 1234 & 1234 & 1234 & 1234 & 1234 \\
\hline $\begin{array}{l}\text { Heal th and radiological safety } \\
\text { impacts and benefits }\end{array}$ & 1234 & 1234 & 1234 & 1234 & 1234 \\
\hline Heal th physics & 1234 & 1234 & 1234 & 1234 & 1234 \\
\hline Safety and health & 1234 & 1234 & 1234 & 1234 & 1234 \\
\hline
\end{tabular}


PART 2 - IDEAL SKILLS FOR EACH TASK

\begin{tabular}{|c|c|c|c|c|c|}
\hline$\frac{\text { TASKS } \rightarrow>}{\text { SXILLS/KMOULEDGE }}$ & $\begin{array}{c}\text { INTERFACE } \\
\text { MITHIM/OUTSIDE DOE } \\
\end{array}$ & $\begin{array}{c}\text { ADVISE, ASSIST } \\
\text { PROVIDE } \\
\text { MUHORITATIVE } \\
\text { CUIDAMCE } \\
\end{array}$ & $\begin{array}{l}\text { PROVIDE REQULATORY } \\
\text { AHALYSIS } 2 \text { CUIDANCE } \\
\end{array}$ & $\begin{array}{c}\text { PRONIDE SUPPORT TO } \\
\text { COWDUCI } \\
\text { PERAIITIMG/LICENSING } \\
\text { ACUNUUIES } \\
\end{array}$ & $\begin{array}{c}\text { IMPLENEHT } \\
\text { REGULATIONS } 4 \\
\text { POLICY } \\
\end{array}$ \\
\hline Iransportation issues & 1234 & 1234 & 1234 & 1234 & 1234 \\
\hline Packaging issues & 1234 & 1234 & 1234 & 12334 . & $1234 \ldots$ \\
\hline Biology, zoology, and ecology & 1234 & 1234 & 1234 & 1234 & 1234 \\
\hline Chemistry & 1234 & 1234 & 1234 & 1234 & 1234 \\
\hline Nuclear engineering & 1234 & 1234 & 1234 & 1234 & 1234 \\
\hline Physical sciences & 1234 & 1234 & 1234 & 1234 & 1234 \\
\hline Geohydrology & 1234 & 1234 & 1234 & 1234 & $1234=$ \\
\hline Cartography & 1234 & 1234 & 1234 & 1234 & 1234 \\
\hline Geophysics & 1234 & 1234 & 1234 & 1234 & 1234 \\
\hline D80 techniques & 1234 & 1234 & 1234 & 1234 & 1234 \\
\hline Cultural diversity issues & 1234 & 1234 & 1234 & 1234 & 1234 \\
\hline $\begin{array}{l}\text { Hazardaus, chemical, radioact ive } \\
\text { and mixed waste management }\end{array}$ & 1234 & 1234 & 1234 & 1234 & 1234 \\
\hline RCRA/CERCLA process & 1234 & 1234 & 1234 & 1234 & 1234 \\
\hline Natural resources & 1234 & $\begin{array}{llll}1 & 2 & 3 & 14 \\
\end{array}$ & 1234 & 1234 & 1234. \\
\hline Lend use & 1234 & 1234 & 1234 & 1234 & 1234 \\
\hline Hildlife management & 1234 & 1234 & 1234 & 1234 & 1234 \\
\hline Soils, and minerols & 1234 & 1234 & 1234 & 1234 & 1234 \\
\hline Aquatic resources & 1234 & 1234 & 1234 & 1234 & 1234 \\
\hline NEPA process & 1234 & 1234 & $12 \quad 3 \quad 4$ & 1234 & 1234 \\
\hline Enviromental sciences & 1234 & 1234 & 1234 & 1234 & 1234 \\
\hline Qual ity assurance & 1234 & 1234 & 1234 & 1234 & $1234=-$ \\
\hline Emergency response procedures & 1234 & 1234 & 1234 & 1234 & 1234 \\
\hline
\end{tabular}


PART 2 - IDEAL SKILLS FOR EACH TASK

\begin{tabular}{|c|c|c|c|c|c|}
\hline$\frac{\text { TASKS } \cdots>}{\text { SKILLS/KWOULEDGE }}$ & $\begin{array}{l}\text { OPERATE EQUIPKERT } \\
\text { (E.G., COHPUIERS, FAX, } \\
\text { cOPY MCHINES) } \\
\end{array}$ & $\begin{array}{c}\text { MAKACE FILES \& } \\
\text { RECORDS } \\
\end{array}$ & $\begin{array}{c}\text { IRACK } \\
\text { CORRESPOWEENCE } \\
\end{array}$ & $\begin{array}{l}\text { PERFORA TRAVEL } \\
\text { NOWINISTRAYIVE } \\
\text { FUWCTIOWS } \\
\end{array}$ & $\begin{array}{c}\text { OVERSEE } \\
\text { DATNINFORMATIOA } \\
\text { MUACEEENT (E.G.. } \\
\text { OOPS) } \\
\end{array}$ \\
\hline National consensus standards & 1234 & 1234 & 1234 & 1234 & 1234 \\
\hline $\begin{array}{l}\text { Interagency agreements, } \\
\text { administrotion, and process }\end{array}$ & 1234 & 1234 & 1234 & 1234 & 1234 \\
\hline $\begin{array}{l}\text { Organizing information and present } \\
\text { cogent orguments }\end{array}$ & $123 \quad 4$ & 1234 & 1234 & 1234 & 1234 \\
\hline Writing succinctly and clearly & 1234 & 1234 & 1234 & 1234 & 1234 \\
\hline $\begin{array}{l}\text { Public speaking (e.g.., public } \\
\text { presentations, employee/employer } \\
\text { interactions) }\end{array}$ & 1234 & 1234 & 1234 & 1234 & 1234 \\
\hline $\begin{array}{l}\text { Interpersonal facility (e.g., } \\
\text { public, medio, other employees and } \\
\text { special interest groups) }\end{array}$ & 1234 & 1234 & 1234 & 1234 & 1234 \\
\hline Hegotiating/conflict resolution & 1234 & 1234 & 1234 & 1234 & 1234 \\
\hline $\begin{array}{l}\text { Providing clear guidance and } \\
\text { direction to accomplish tasks }\end{array}$ & 1234 & 1234 & 1234 & 1234 & 1234 \\
\hline Meeting facilitation/teadership & 1234 & 1234 & 1234 & 1234 & 1234 \\
\hline DOE management strategies & $123 \quad 4$ & 1234 & 1234 & 1234 & 1234 \\
\hline $\begin{array}{l}\text { EH's mission, policies, management } \\
\text { plans, procedures, priorities, goals } \\
\text { and objectives }\end{array}$ & 1234 & $\begin{array}{r}1 \\
1234 \\
\end{array}$ & 1234 & 1234 & 1234 \\
\hline Decision enalysis & 1234 & 1234 & 1234 & 1234 & 1234 \\
\hline Conducting library research & 1234 & 1234 & 1234 & 11234 & 1234 \\
\hline $\begin{array}{l}\text { Facility, site, operations and } \\
\text { processes }\end{array}$ & 1234 & 1234 & $123 \quad 3 \quad 4$ & 1234 & 1234 \\
\hline Defining boundaries of a problem & 1234 & 1234 & 1234 & 1234 & 1234 \\
\hline $\begin{array}{l}\text { Keeping abreast of current } \\
\text { developments }\end{array}$ & 1234 & 1234 & 1234 & 1234 & 1234 \\
\hline Organizing/categorizing & 1234 & 1234 & 1234 & 1234 & 1234 \\
\hline $\begin{array}{l}\text { Operating equipment (e.g., testing } \\
\text { equipment, respirators) }\end{array}$ & 1234 & 1234 & 1234 & 1234 & 1234 \\
\hline Operating and using computer & 1234 & 1234 & 1234 & 1234 & $\begin{array}{llll}1 & 2 & 3 & 4\end{array}$ \\
\hline
\end{tabular}


PART 2 - IDEAL SKILLS FOR EACH TASK

\begin{tabular}{|c|c|c|c|c|c|}
\hline$\frac{\text { IASKS }-\cdots}{\text { SKILLS/KMOULEDCE }}$ & $\begin{array}{c}\text { OPERATE EQUIPHENT } \\
\text { (E.G., COMPUIERS, FAX, } \\
\text { COPY MACHIMES) } \\
\end{array}$ & $\begin{array}{c}\begin{array}{c}\text { MUACE FILES } 2 \\
\text { RECORDS }\end{array} \\
\end{array}$ & $\begin{array}{c}\text { TRACX } \\
\text { CORRESPONOENCE } \\
\end{array}$ & $\begin{array}{l}\text { PERFCRN TRAVEL } \\
\text { ADWINISTRAIIIVE } \\
\text { FUWCIIONS } \\
\end{array}$ & $\begin{array}{c}\text { ONERSEE } \\
\text { DATNIMFORMTION } \\
\text { MUMACENEMT (E.G.., } \\
\text { OQPS) } \\
\end{array}$ \\
\hline Touch typing & 1234 & 1234 & 1234 & 1234 & 1234 \\
\hline $\begin{array}{l}\text { Understanding principles of data } \\
\text { base requirements and baseline } \\
\text { techniques }\end{array}$ & 1234 & 1234 & 1234 & 1234 & 1234 \\
\hline $\begin{array}{l}\text { Using software and applying it to } \\
\text { tasks }\end{array}$ & 1234 & 1234 & 1234 & 1234 & 1234 \\
\hline rime management & 1234 & 1234 & 1234 & 1234 & 1234 \\
\hline Heighing cost versus benefits & 1234 & 1234 & 1234 & 1234 & 1234 \\
\hline $\begin{array}{l}\text { Project or program management } \\
\text { techniques }\end{array}$ & 1234 & 1234 & 1234 & 1234 & 1234 \\
\hline $\begin{array}{l}\text { Principles of DOE budget } \\
\text { formulations, budget execution and } \\
\text { cost accounting, and the procedures } \\
\text { involved }\end{array}$ & 1234 & 1234 & 1234 & 1234 & 1234 \\
\hline Schedule constraints & 1234 & 1234 & 1234 & 1234 & 1234 \\
\hline $\begin{array}{l}\text { Planning, programing, funding and } \\
\text { acquisition management }\end{array}$ & 1234 & 1234 & 1234 & 1234 & 1234 \\
\hline $\begin{array}{l}\text { Conduct ing analyses involving } \\
\text { complex variables }\end{array}$ & 1234 & $122^{1} 34$ & 1234 & 1234 & 1234 \\
\hline Supervising staff/contractors & 1234 & 1234 & 1234 & 1234 & 1234 \\
\hline $\begin{array}{l}\text { Delegating authority and } \\
\text { responsibility }\end{array}$ & 1234 & 1234 & 1234 & 1,234 & 1234 \\
\hline Equal Opportunity program plans & 1234 & 1234 & 1234 & 1.234 & 1234 \\
\hline $\begin{array}{l}\text { Analyzing strengths and Heaknesses } \\
\text { of al ternatives }\end{array}$ & 1234 & 1234 & 1234 & 1234 & 1234 \\
\hline Anticipating unintended consequences & 1234 & 1234 & 1234 & 1234 & 1234 \\
\hline $\begin{array}{l}\text { Comparing existing conditions to } \\
\text { pre-set standards }\end{array}$ & 1234 & 1234 & 1234 & 1234 & 1234 \\
\hline Prioritizing based on criteria & 1234 & 1234 & 1234 & 1234 & 1234 \\
\hline $\begin{array}{l}\text { Identifying relevant } \\
\text { material/requirements }\end{array}$ & 1234 & 1234 & 1234 & 1234 & 1234 \\
\hline
\end{tabular}


PART 2 - IDEAL SKILLS FOR EACH TASK

\begin{tabular}{|c|c|c|c|c|c|}
\hline$\frac{\text { TASKS } \rightarrow \rightarrow}{\text { SKILLS/KMOMLEDGE }}$ & $\begin{array}{c}\text { OPERATE EQUIPHEHT } \\
\text { (E.G., COWPUTERS, FAX, } \\
\text { COPY MACHIHES) } \\
\end{array}$ & $\begin{array}{l}\text { MURACE FILES \& } \\
\text { RECORDS } \\
\end{array}$ & $\begin{array}{c}\text { TRACK } \\
\text { CORRESPONOENCE } \\
\end{array}$ & $\begin{array}{c}\text { PERFORA TRAVEL } \\
\text { ROMIMISTRATIVE } \\
\text { FUWCIIONS } \\
\end{array}$ & $\begin{array}{c}\text { OVERSEE } \\
\text { DATNINFORMATION } \\
\text { MUMACENENI (E.G.. } \\
\text { OOES) } \\
\end{array}$ \\
\hline Delegating tosks & 1234 & 1234 & 1234 & 1234 & 1234 \\
\hline Defining applicable criteria & 1234 & 1234 & 1234 & 1234 & 1234 \\
\hline $\begin{array}{l}\text { Reading technical material for a set } \\
\text { purpose }\end{array}$ & 1234 & 1234 & 1234 & 1234 & 1234 \\
\hline Speed reading & 1234 & 1234 & 1234 & 1234 & 1234 \\
\hline Consol idating materiots & 1234 & 1234 & 1234 & 1234 & 1234 \\
\hline $\begin{array}{l}\text { Examining trade-offs among } \\
\text { al ternatives }\end{array}$ & 1234 & 1234 & 1234 & 1234 & 1234 \\
\hline $\begin{array}{l}\text { Geomapping, remote sensing, and } \\
\text { characterization techniques }\end{array}$ & 1234 & 1234 & 1234 & 1234 & 1234 \\
\hline $\begin{array}{l}\text { Proper engineering/technical } \\
\text { stonderds }\end{array}$ & 1234 & 1234 & 1234 & 1234 & 1234 \\
\hline Valid engineering solutions & $123 \quad 34$ & 1234 & 1234 & 1234 & 1234 \\
\hline Remediation techniques & 1234 & 1234 & 1234 & 1234 & 1234 \\
\hline $\begin{array}{l}\text { Waste treatment/disposal } \\
\text { technologies }\end{array}$ & $\begin{array}{llll}1 & 2 & 3 & 4 \\
\end{array}$ & 1234 & 1234 & 1234 & 1234 \\
\hline Human heal th impacts & 1234 & 1234 & 1234 & 1234 & 1234 \\
\hline Radiation effects & 1234 & 1234 & 1234 & 1234 & 1234 \\
\hline $\begin{array}{l}\text { Heal th and radiological safety } \\
\text { impacts and benefits }\end{array}$ & 1234 & 1234 & 1234 & 1234 & 1234 \\
\hline Heal th physics & 1234 & 1234 & 1234 & 1.234 & 1234 \\
\hline Safety and heal th & 1234 & 1234 & 1234 & 1234 & 1234 \\
\hline Iransportation issues & 1234 & 1234 & 1234 & 1234 & 1234 \\
\hline Packoging issues & 1234 & 1234 & 1234 & 1234 & 1234 \\
\hline Biology, zoology, and ecology & 1234 & 1234 & $123 \quad 4$ & 1234 & 1234 \\
\hline Chemistry & 1234 & 1234 & 1234 & 1234 & 1234 \\
\hline Nuclear engineering & 1234 & 1234 & $123 \quad 3$ & 1234 & 1234 \\
\hline Physical sciences & $\begin{array}{llll}1 & 2 & 3 & 4\end{array}$ & 1234 & 1234 & 1234 & 1234 \\
\hline
\end{tabular}


PART 2 - IDEAL SKILLS FOR EACH TASK

\begin{tabular}{|c|c|c|c|c|c|}
\hline IASKS -.) & $\begin{array}{l}\text { OPERAIE EQUIPNEHT } \\
\text { (E.G., COMPUTERS, FAX, } \\
\text { COPY MACHINES) } \\
\end{array}$ & $\begin{array}{c}\begin{array}{c}\text { MUUACE FILES } \\
\text { RECOKDS }\end{array} \\
\end{array}$ & $\begin{array}{c}\text { IRACX } \\
\text { CORRESPOWDENCE } \\
\end{array}$ & $\begin{array}{l}\text { PERFORM YRAVEL } \\
\text { NOHIMISYRAYIVE } \\
\text { FUWCTIOWS } \\
\end{array}$ & $\begin{array}{c}\text { OVERSEE } \\
\text { DATNINFORMAIION" } \\
\text { MUKAGEMENT (E.G.." } \\
\text { ORPS) }\end{array}$ \\
\hline Geohydrology & 1234 & 1234 & 1234 & 1234 & 1234 \\
\hline Cartography & 1234 & 1234 & 1234 & 1234 & 1234 \\
\hline Geophysics & 1234 & $\begin{array}{llll}1 & 2 & 3 & 4\end{array}$ & $\begin{array}{llll}1 & 2 & 3 & 4\end{array}$ & 1234 & 1234 \\
\hline 080 techniques & 1234 & 1234 & 1234 & 1234 & 1234 \\
\hline Cultural diversity issues & 1234 & 1234 & 1234 & 1234 & 1234 \\
\hline $\begin{array}{l}\text { Hazordous, chenicul, radiouct lve } \\
\text { and mixed haste managentent }\end{array}$ & 1234 & 1234 & 1234 & 1234 & 1234 \\
\hline RCRA/CERCLA process & 1234 & 1234 & 1234 & 1234 & 1234 \\
\hline Natural resources & 1234 & 1234 & 1234 & 1234 & 1234 \\
\hline Land use & 1234 & 1234 & 1234 & 1234 & 1234 \\
\hline Hildlife management & 1234 & 1234 & 1234 & 1234 & 1234 \\
\hline Soils, and minerals & 1234 & 1234 & 1234 & 1234 & 1234 \\
\hline Aquat ic resources & 1234 & 1234 & 1234 & 1234 & 1234 \\
\hline NEPA process & 1234 & 1234 & $\begin{array}{llll}1 & 2 & 3 & 4\end{array}$ & 1234 & 1234 \\
\hline Enviromental sciences & 1234 & 1234 & 1234 & 1234 & 1234 \\
\hline Quality assurance & 1234 & $123 \quad 34$ & 1234 & $123 \quad 3$ & 1234 \\
\hline Emergency response procedures & $\begin{array}{llll}1 & 2 & 3 & 4 \\
\end{array}$ & 1234 & 1234 & 1234 & 1234 \\
\hline
\end{tabular}


PART 2 - IDEAL SKILLS FOR EACH TASK

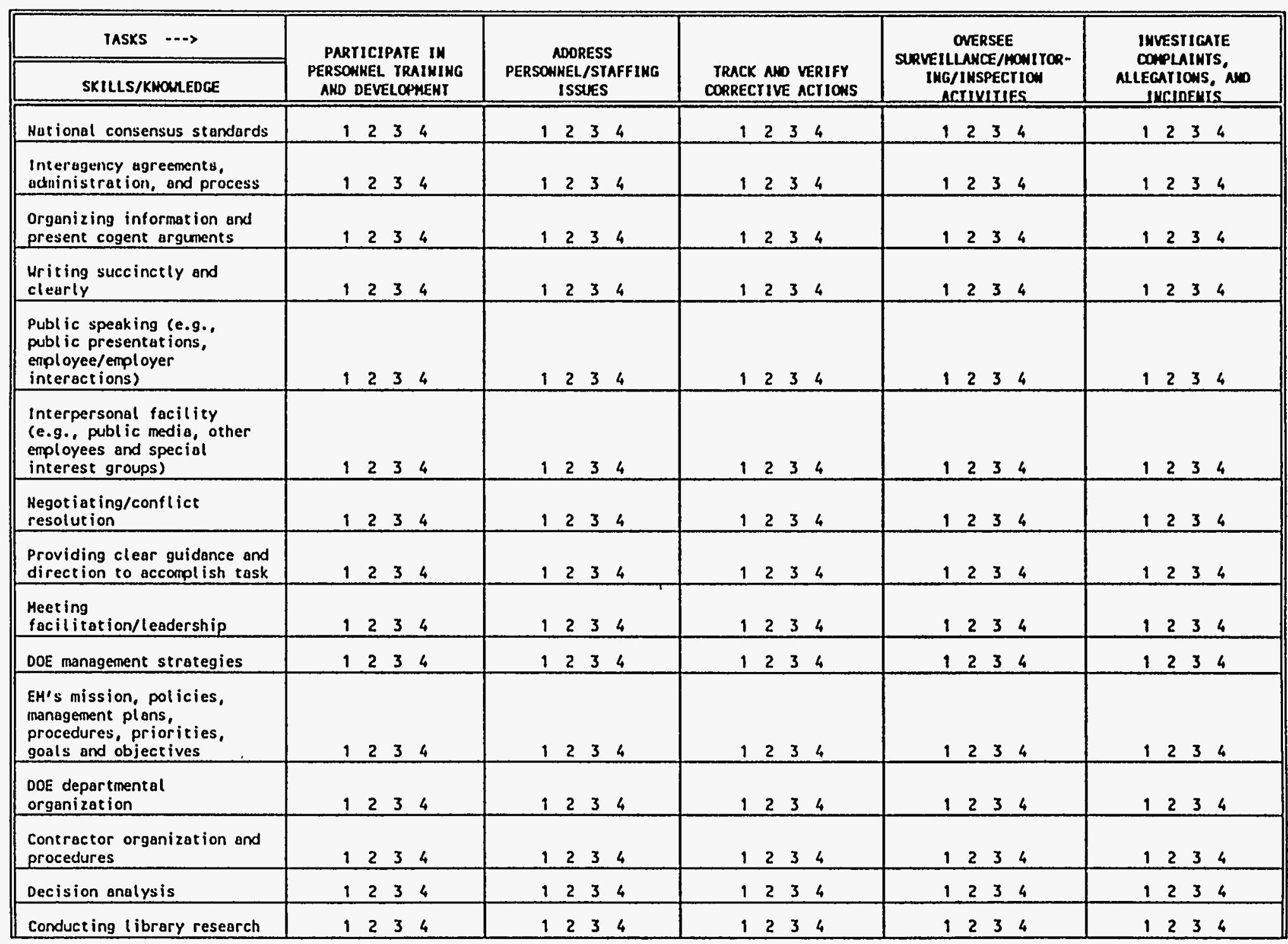


PART 2 - IDEAL SKILLS FOR EACH TASK

\begin{tabular}{|c|c|c|c|c|c|}
\hline$\frac{\text { TASXS } \cdots>}{\text { SXILLS/XNOULEDCE }}$ & $\begin{array}{l}\text { PARTICIPATE IM } \\
\text { PERSOWHEL TRAINIMG } \\
\text { AND DEVELOPHENT } \\
\end{array}$ & $\begin{array}{l}\text { ADORESS } \\
\text { PERSOWKEL/STAFFING } \\
\text { ISSUES } \\
\end{array}$ & $\begin{array}{l}\text { TRACK ANO VERIFY } \\
\text { CORKECIIVE ACIIOWS }\end{array}$ & $\begin{array}{c}\text { OVERSEE } \\
\text { SURVEILLLWCE/HONITOR- } \\
\text { ING/INSPECIION } \\
\text { ACIIYIIIES } \\
\end{array}$ & $\begin{array}{c}\text { IMVESTIGATE } \\
\text { COMULAINTS, } \\
\text { ALLEGATIONS, ANO } \\
\text { IMCIOEMIS } \\
\end{array}$ \\
\hline $\begin{array}{l}\text { Fucility, site operations } \\
\text { and processes }\end{array}$ & 9.234 & 1234 & 12.34 & 1234 & 1234 \\
\hline $\begin{array}{l}\text { Defining bourdaries of a } \\
\text { problemn }\end{array}$ & 1234 & 1234 & 1234 & 1234 & 1234 \\
\hline $\begin{array}{l}\text { Keeping abreast of current } \\
\text { developments }\end{array}$ & 1234 & 1234 & 1234 & 1234 & 1234 \\
\hline Organizing/categorizing & 1234 & 1234 & 1234 & 1234 & 1234 \\
\hline $\begin{array}{l}\text { Operating equipment (e.g., } \\
\text { testing equipment, } \\
\text { respirators) }\end{array}$ & 1234 & 1234 & 1234 & 123.4 & 1234 \\
\hline Operating and using computer & 1234 & 1234 & 1234 & 1234 & 1234 \\
\hline rouch typing & 1234 & 1234 & 1234 & 1234 & 1234 \\
\hline $\begin{array}{l}\text { Understanding principles of } \\
\text { data base requirements and } \\
\text { basel ine techniques }\end{array}$ & 1234 & 1234 & 1234 & 1234 & 1234 \\
\hline $\begin{array}{l}\text { Using software and applying } \\
\text { it to tasks }\end{array}$ & 1234 & 1234 & 1234 & 1234 & 1234 \\
\hline Time management & 1234 & $\begin{array}{lllll}1 & 2 & 3 & 4 & 1 \\
\end{array}$ & 1234 & 1234 & 1234 \\
\hline $\begin{array}{l}\text { Heighing costs versus } \\
\text { benefits }\end{array}$ & 1234 & 1234 & 1234 & 1234 & 1234 \\
\hline $\begin{array}{l}\text { Project or program } \\
\text { management techniques }\end{array}$ & 1234 & 1234 & 1234 & 1234 & 1234 \\
\hline $\begin{array}{l}\text { Principles of DoE budget } \\
\text { formulations, budget } \\
\text { execution and cost } \\
\text { accounting, and the } \\
\text { procedures involved }\end{array}$ & 1234 & 1234 & 1234 & 1234 & 1234 \\
\hline Schedule constraints & 1234 & 1234 & 1234 & 1234 & 1234 \\
\hline $\begin{array}{l}\text { Planning, programming, } \\
\text { funding and acquisition } \\
\text { manogement }\end{array}$ & 1234 & 1234 & 1234 & 1234 & 1234 \\
\hline $\begin{array}{l}\text { Conducting analyses } \\
\text { involving complex variables }\end{array}$ & 1234 & 1234 & 1234 & $\begin{array}{llll}1 & 2 & 3 & 4\end{array}$ & 1234 \\
\hline
\end{tabular}


PART 2 - IDEAL SKILLS FOR EACH TASK

\begin{tabular}{|c|c|c|c|c|c|}
\hline$\frac{\text { IASKS } \rightarrow-\text { > }}{\text { SKILLS/KHOAR.EDGE }}$ & $\begin{array}{l}\text { PARTICIPATE IK } \\
\text { PERSOWNEL TRAINIKG } \\
\text { ARD DEEVELOPHEMT } \\
\end{array}$ & $\begin{array}{c}\text { AODRESS } \\
\text { PERSOWHEL/STAFFING } \\
\text { ISSUES } \\
\end{array}$ & $\begin{array}{l}\text { TRACK AND VERIFY } \\
\text { CORRECTIVE ACTIOHS } \\
\end{array}$ & $\begin{array}{l}\text { OVERSEE } \\
\text { SURVEILLANCE/MOWI TOR- } \\
\text { ING/INSPECTIOW } \\
\text { ACTIVINES } \\
\end{array}$ & $\begin{array}{l}\text { INVESTIGATE } \\
\text { COWHLAINIS, } \\
\text { ALLEGATIONS, NVO } \\
\text { IMCIDEYIS } \\
\end{array}$ \\
\hline stuff/controctors & 1234 & 1234 & $123 \quad 3$ & 1234 & 1234 \\
\hline $\begin{array}{l}\text { Delegating authority and } \\
\text { responsibility }\end{array}$ & 1234 & 1234 & 1234 & 1234 & 1234 \\
\hline $\begin{array}{l}\text { Equal Opportunity progrum } \\
\text { plans }\end{array}$ & 1234 & 1234 & 1234 & 1234 & 1234 \\
\hline $\begin{array}{l}\text { Anulyzing/synthesizing } \\
\text { information }\end{array}$ & 1234 & 1234 & 1234 & 1234 & 1234 \\
\hline $\begin{array}{l}\text { Assessing strengths and } \\
\text { Heaknesses of al ternatives }\end{array}$ & 1234 & 1234 & 1234 & 1234 & $123 \quad 4$ \\
\hline $\begin{array}{l}\text { Anticipating unintended } \\
\text { consequences }\end{array}$ & $\begin{array}{llll}1 & 2 & 3 & 4\end{array}$ & 1234 & 1234 & 1234 & 1234 \\
\hline $\begin{array}{l}\text { Comparing existing } \\
\text { conditions to pre-set } \\
\text { standards }\end{array}$ & 1234 & 1234 & 1234 & 1234 & 1234 \\
\hline $\begin{array}{l}\text { Prioritizing based on } \\
\text { criteria }\end{array}$ & 1234 & 1234 & 1234 & $123 \quad 34$ & 1234 \\
\hline $\begin{array}{l}\text { Identifying relevant } \\
\text { material/requirements }\end{array}$ & $123 \quad 34$ & 1234 & 1234 & 1234 & 1234 \\
\hline Delegating tasks & 1234 & 1234 & 1234 & 1234 & 1234 \\
\hline Defining applicable criteria & 1234 & 1234 & 1234 & 1234 & 1234 \\
\hline $\begin{array}{l}\text { Reuding technical material } \\
\text { for a set purpose }\end{array}$ & $\begin{array}{llll}1 & 2 & 3 & 4 \\
\end{array}$ & 1234 & 1234 & 1234 & 1234 \\
\hline Speed reading & $123 \quad 3 \quad 4$ & $123 \quad 34$ & 1234 & 1234 & 1234 \\
\hline Consolidating material & 1234 & 1234 & 1234 & 1234 & 1234 \\
\hline $\begin{array}{l}\text { Examining trade-ofts anong } \\
\text { ol ternatives }\end{array}$ & $123 \quad 34$ & 1234 & 1234 & 1234 & 1234 \\
\hline $\begin{array}{l}\text { Geomapping, remote sensing, } \\
\text { and characterization } \\
\text { techniques }\end{array}$ & 1234 & 1234 & 1234 & 1234 & 1234 \\
\hline $\begin{array}{l}\text { Proper engineering/technical } \\
\text { standards }\end{array}$ & 1234 & 1234 & 1234 & 1234 & 1234 \\
\hline
\end{tabular}


PART 2 - IDEAL SKILLS FOR EACH TASK

\begin{tabular}{|c|c|c|c|c|c|}
\hline $\begin{array}{c}\text { IASKS } \cdots \text { - } \\
\text { SKILLS/KMOULEOGE }\end{array}$ & $\begin{array}{l}\text { PARTICIPATE IN } \\
\text { PERSOWNEL TRAINIMG } \\
\text { AHD DEVELOPMEHI } \\
\end{array}$ & $\begin{array}{c}\text { ADORESS } \\
\text { PERSOWMEL/SIAFFING } \\
\text { ISSUES } \\
\end{array}$ & $\begin{array}{l}\text { IRACK AND VERIFY } \\
\text { OORRECTIVE ACIIOWS } \\
\end{array}$ & $\begin{array}{c}\text { OVERSEE } \\
\text { SURVEILLANCE/HOWITOR- } \\
\text { ING/INSPECIION } \\
\text { ACUUYUUES } \\
\end{array}$ & 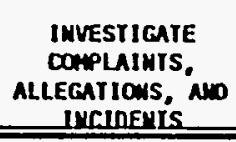 \\
\hline Vulid engineering solutions & 1234 & 1234 & 1234 & 1234 & 1234 \\
\hline Renkediation techniques & 1234 & 1234 & 1234 & 1234 & 1234 \\
\hline $\begin{array}{l}\text { Wuste treatment/disposal } \\
\text { techrologies }\end{array}$ & 1234 & 1234 & $123 \quad 3 \quad 4$ & 1234 & 1234 \\
\hline Himan health impacts & 1234 & 1234 & 1234 & 1234 & 1234 \\
\hline Rudiation effects & 1234 & 1234 & 1234 & 1234 & 1234 \\
\hline $\begin{array}{l}\text { Health and radiological } \\
\text { safety inpacts and benefits }\end{array}$ & 1234 & 1234 & 1234 & 1234 & 1234 \\
\hline Heal th physics & 1234 & 1234 & 1234 & 1234 & 1234 \\
\hline Safety and Health & 1234 & 1234 & 1234 & 1234 & 1234 \\
\hline Irensportation issues & 1234 & 1234 & 1234 & 1234 & 1234 \\
\hline Packaging issues & 1234 & 1234 & 1234 & 1234 & 1234 \\
\hline $\begin{array}{l}\text { Biology, zoology, and } \\
\text { ecology }\end{array}$ & 1234 & 1234 & 1234 & 1234 & $123 \quad 3 \quad 4$ \\
\hline Chemistry & 1234 & 1234 & 1234 & 1234 & 1234 \\
\hline Nuclear engineering & 1234 & 1234 & 1234 & 1234 & 1234 \\
\hline Physical sciences & 1234 & 1234 & 1234 & 1234 & 1234 \\
\hline Geohydrology & 1234 & 1234 & 1234 & 1234 & 1234 \\
\hline Cartography & 1234 & 1234 & 1234 & 1234 & 1234 \\
\hline Geophysics & 1234 & 1234 & 1234 & 1234 & $123 \quad 34$ \\
\hline D8D rechniques & 1234 & 1234 & 1234 & 1234 & 1234 \\
\hline Cultural diversity issues & 1234 & 1234 & $123 \quad 4$ & 1234 & 1234 \\
\hline $\begin{array}{l}\text { Hazardous, chemical, } \\
\text { radiooctive and mixed haste } \\
\text { management }\end{array}$ & 1234 & 1234 & 1234 & 1234 & 1234 \\
\hline RCRA/CERCLA process & 1234 & 1234 & $123 \quad 34$ & 1234 & 1234 \\
\hline Natural resources & 1234 & 1234 & $123 \quad 3 \quad 4$ & 12.34 & 1234 \\
\hline Land use & 1234 & 1234 & $123 \quad 4$ & 1234 & 1234 \\
\hline
\end{tabular}


PART 2 - IDEAL SKILLS FOR EACH TASK

\begin{tabular}{|c|c|c|c|c|c|}
\hline$\frac{\text { IASKS } \cdots \text { - }}{\text { SKILLS/KHOULEDGE }}$ & $\begin{array}{c}\text { PARYICIPATE IN } \\
\text { PERSOWHEL TRAINING } \\
\text { AND DEVELOPHEWT } \\
\end{array}$ & $\begin{array}{c}\text { NODRESS } \\
\text { PERSOWHEL/STAFFIMG } \\
\text { ISSUES } \\
\end{array}$ & $\begin{array}{l}\text { IRACX NO VERIFY } \\
\text { CORRECTIVE ACIIOWS } \\
\end{array}$ & $\begin{array}{c}\text { OVERSEE } \\
\text { SURVEILLAKCE/MOMITOR- } \\
\text { ING/INSPECTIOW } \\
\text { ACTUVUES } \\
\end{array}$ & 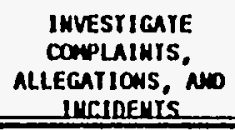 \\
\hline Wildlife management & 1234 & $123 \quad 34$ & 1234 & 1234 & 1234 \\
\hline Soils, and minerals & 1234 & 1234 & 1234 & 1234 & 1234 \\
\hline Aquatic resources & 1234 & 1234 & 1234 & 1234 & 1234 \\
\hline HEPA processs & 1234 & 1234 & 1234 & 1234 & 1234 \\
\hline Environmental sciences & 1234 & 1234 & 1234 & 1234 & 1234 \\
\hline Quality assurance & $123 \quad 4$ & 1234 & 1234 & 1234 & 1234 \\
\hline $\begin{array}{l}\text { Emergency response } \\
\text { procedures }\end{array}$ & 1234 & 1234 & 1234 & 1234 & 1234 \\
\hline
\end{tabular}


PART 2 - IDEAL SKILLS FOR EACH TASK

\begin{tabular}{|c|c|c|c|c|c|}
\hline TASKS $-\rightarrow>$ & $\begin{array}{c}\text { PROVIDE AKALYSES TO } \\
\text { DEIERMINE EFFECTS } \\
\text { OF REREDIATIOW } \\
\text { PROIECIS } \\
\end{array}$ & $\begin{array}{l}\text { REVIEU REMEDIATION } \\
\text { PROJECIS ANO THEIR } \\
\text { PROGRESS } \\
\end{array}$ & $\begin{array}{c}\text { RECOWNEN ACYIOWS } \\
\text { TO COKRECT REMEDIAL } \\
\text { PROQLERS } \\
\end{array}$ & $\begin{array}{c}\text { IDENTIFY NN } \\
\text { DOQWEMT } \\
\text { DEFICIENCIES } \\
\end{array}$ & SET SCHEDULES \\
\hline Nutional consensus standarus & 1234 & 1234 & 1234 & 1234 & 1234 \\
\hline $\begin{array}{l}\text { Interagency agreements, } \\
\text { adninistration, and process }\end{array}$ & 1234 & 1234 & 1234 & 1234 & 1234 \\
\hline $\begin{array}{l}\text { Organizing information and present } \\
\text { cogent orguments }\end{array}$ & 1234 & 1234 & 1234 & 1234 & 1234 \\
\hline Writing succinctly and clearly & 1234 & 1234 & 1234 & 1234 & 1234 \\
\hline $\begin{array}{l}\text { Public speaking (e.9., public } \\
\text { presentations, enployee/entployer }\end{array}$ & 1234 & 1234 & 1234 & 1234 & 1234 \\
\hline $\begin{array}{l}\text { Interpersonal facility (e.g., } \\
\text { public, media, other employees and } \\
\text { special interest groups) }\end{array}$ & 1234 & 1234 & 1234 & 1234 & 1234 \\
\hline Hegotiating/conflict resolution & 1234 & 1234 & 1234 & 1234 & 1234 \\
\hline $\begin{array}{l}\text { Providing clear guidance and } \\
\text { direction to accompl ish tasks }\end{array}$ & 1234 & 1234 & 1234 & 1234 & 1234 \\
\hline Heeting facilitation/leadership & 1234 & 1234 & 1234 & 1234 & 1234 \\
\hline DOE management strategies & 1234 & 1234 & 1234 & 1234 & 1234 \\
\hline $\begin{array}{l}\text { EM's mission, policies, management } \\
\text { plans, procedures, priorities, } \\
\text { goals and objectives }\end{array}$ & 1234 & 1234 & 1234 & 1234 & 1234 \\
\hline DOE departmental organization & 1234 & 1234 & 1234 & 1234 & 1234 \\
\hline $\begin{array}{l}\text { Contractor organization and } \\
\text { procedures }\end{array}$ & $123 \quad 4$ & 1234 & 1234 & $\begin{array}{llll}1 & 2 & 3 & 4 \\
\end{array}$ & 1234 \\
\hline Decision analysis & 1234 & 1234 & 1234 & 1234 & 1234 \\
\hline Conducting library research & 1234 & 1234 & 1234 & 1234 & 1234 \\
\hline $\begin{array}{l}\text { Facility, site, operations and } \\
\text { processes }\end{array}$ & 1234 & 1234 & 1234 & 1234 & 1234 \\
\hline Defining boundaries of a problem & 1234 & 1234 & 1234 & 1234 & 1234 \\
\hline $\begin{array}{l}\text { Keeping abreast of current } \\
\text { developments }\end{array}$ & 1234 & 1234 & 1234 & 1234 & 1234 \\
\hline Organizing/categorizing & 1234 & 1234 & 1234 & 1234 & 1234 \\
\hline
\end{tabular}


PART 2 - IDEAL SKILLS FOR EACH TASK

\begin{tabular}{|c|c|c|c|c|c|}
\hline$\frac{\text { rASKS } \cdots \text {-.) }}{\text { SKILLS/KHOAR.EOCE }}$ & $\begin{array}{c}\text { PROVIDE MHALYSES YO } \\
\text { DETERMIME EFFECTS } \\
\text { OF REHEDIATIOW } \\
\text { PROIECIS } \\
\end{array}$ & $\begin{array}{c}\text { REVIEU REHEOIATION } \\
\text { PROJECTS NO YHEIR } \\
\text { PROGRESS } \\
\end{array}$ & $\begin{array}{c}\text { RECOAWELD ACTIOWS } \\
\text { IO CORRECT REHEDIAL } \\
\text { PROALEMS } \\
\end{array}$ & $\begin{array}{c}\text { IDENYIFY ANO } \\
\text { DOQWEMT } \\
\text { DEFICIENCIES } \\
\end{array}$ & SET SCHEDULES \\
\hline $\begin{array}{l}\text { Operating equipment (e.9., testing } \\
\text { equipakent, respirotors) }\end{array}$ & 1234 & 1234 & 1234 & 1234 & 1234 \\
\hline Operating and using computer & 1234 & 1234 & 1234 & $\begin{array}{llll}1 & 2 & 3 & 4\end{array}$ & $\begin{array}{llll}1 & 2 & 3 & 4\end{array}$ \\
\hline Touch typing & 1234 & 1234 & 1234 & 1234 & 1234 \\
\hline $\begin{array}{l}\text { Understunding principles of duto } \\
\text { base requir rements and basel ine } \\
\text { tuchnigues }\end{array}$ & 1234 & $1234^{\prime}$ & 1234 & 1234 & 1234 \\
\hline $\begin{array}{l}\text { Using sof tware and applying it to } \\
\text { tasks }\end{array}$ & 1234 & $123 \quad 4$ & $123 \quad 4$ & 1234 & 1234 \\
\hline I ille manugement & 1234 & 1234 & 1234 & 1234 & 1234 \\
\hline Heighing costs versus benefits & 1234 & 1234 & 1234 & 1234 & 1234 \\
\hline $\begin{array}{l}\text { Project or program management } \\
\text { techniques }\end{array}$ & 1234 & 1234 & 1234 & 1234 & 1234 \\
\hline $\begin{array}{l}\text { Principles of DOE budget } \\
\text { formulations, budget execution and } \\
\text { cost account ing, and the } \\
\text { procedures involved }\end{array}$ & 1234 & 1234 & 1234 & 1234 & 1234 \\
\hline Schedule constraints & 1234 & 1234 & 1234 & 1234 & 1234 \\
\hline $\begin{array}{l}\text { Planning, programing, funding and } \\
\text { acquisition managenent }\end{array}$ & $123 \quad 4$ & 1234 & 1234 & 1234 & 1234 \\
\hline $\begin{array}{l}\text { Conducting analyses involving } \\
\text { conplex variables }\end{array}$ & 1234 & 1234 & 1234 & 1234 & 1234 \\
\hline Supervising staff/contractors & 1234 & 1234 & 1234 & 1234 & 1234 \\
\hline $\begin{array}{l}\text { Delegating authority and } \\
\text { responsibility }\end{array}$ & 1234 & $123 \quad 34$ & $123 \quad 3 \quad 4$ & 1234 & 1234 \\
\hline Equal Opportunity program plans & 1234 & 1234 & 1234 & 1234 & 1234 \\
\hline Analyzing/synthesizing information & 1234 & 1234 & 1234 & 1234 & 1234 \\
\hline $\begin{array}{l}\text { Assessing strengths and Heaknesses } \\
\text { of alternatives }\end{array}$ & $\begin{array}{llll}1 & 2 & 3 & 4 \\
\end{array}$ & 1234 & 1234 & 1234 & 1234 \\
\hline $\begin{array}{l}\text { Anticipoting unintended } \\
\text { consequences }\end{array}$ & 1234 & 1234 & 1234 & 1234 & 1234 \\
\hline
\end{tabular}


PART 2 - IDEAL SKILLS FOR EACH TASK

\begin{tabular}{|c|c|c|c|c|c|}
\hline$\frac{\text { TASKS } \cdots>}{\text { SKILLS/KMOULEOCE }}$ & $\begin{array}{l}\text { PROVIDE ANALYYSES TO } \\
\text { DEIERAINE EFFECTS } \\
\text { OF REREDIATION } \\
\text { PROAECIS } \\
\end{array}$ & $\begin{array}{c}\text { REVIEU REMEDIATION } \\
\text { PROJECIS ANO THEIR } \\
\text { PROGRESS } \\
\end{array}$ & $\begin{array}{c}\text { RECOWHENO ACTIOWS } \\
\text { TO CORRECT REMEDIAL } \\
\text { PROBLEMS } \\
\end{array}$ & $\begin{array}{l}\text { IOEMTIFY NO } \\
\text { DOANEMT } \\
\text { DEFICIENCIES }\end{array}$ & SET SCHEDULES \\
\hline $\begin{array}{l}\text { Comporing existing conditions to } \\
\text { pre-set standards }\end{array}$ & 1234 & 1234 & 1234 & 1234 & $\begin{array}{llll}1 & 2 & 3 & 4 \\
\end{array}$ \\
\hline Prioritizing based on criteria & 1234 & 1234 & 1234 & 1234 & 1234 \\
\hline $\begin{array}{l}\text { Ident ifying relevent } \\
\text { muterial/requirenents }\end{array}$ & 1234 & 1234 & 1234 & 1234 & 1234 \\
\hline Delegoting tosks & 1234 & 1234 & 1234 & 1234 & 1234 \\
\hline Defining applicable criteria & 1234 & 1234 & 1234 & 1234 & 1234 \\
\hline $\begin{array}{l}\text { Reading technical material for a } \\
\text { set purpose }\end{array}$ & 1234 & 1234 & 1234 & 1234 & 1234 \\
\hline Speed reading & 1234 & 1234 & 1234 & 1234 & 1234 \\
\hline Consolidating materials & $\begin{array}{llll}1 & 2 & 3 & 4 \\
\end{array}$ & 1234 & 1234 & 1234 & 1234 \\
\hline $\begin{array}{l}\text { Examining trade-offs among } \\
\text { al ternatives }\end{array}$ & 1234 & 1234 & 1234 & 1234 & 1234 \\
\hline $\begin{array}{l}\text { Geomapping, remote sensing, and } \\
\text { characterization techniques }\end{array}$ & 1234 & 1234 & 1234 & $123 \quad 4$ & 1234 \\
\hline $\begin{array}{l}\text { Proper engineering/technical } \\
\text { standerds }\end{array}$ & 1234 & 1234 & 1234 & 1234 & 1234 \\
\hline Valid engineering solutions & 1234 & 1234 & 1234 & 1234 & 1234 \\
\hline Remediation techniques & 1234 & 1234 & 1234 & 1234 & 1234 \\
\hline $\begin{array}{l}\text { Haste treatment/disposal } \\
\text { technologies }\end{array}$ & $\begin{array}{llll}1 & 2 & 3 & 4 \\
\end{array}$ & 1234 & 1234 & 1234 & 1234 \\
\hline Human heal th impacts & 1234 & 1234 & 1234 & 1234 & 1234 \\
\hline Radiation effects & 1234 & 1234 & 1234 & 1234 & 1234 \\
\hline $\begin{array}{l}\text { Heal th and radiological safety } \\
\text { impacts and benefits }\end{array}$ & 1234 & 1234 & 1234 & 1234 & 1234 \\
\hline Heal th physics & 1234 & 1234 & 1234 & 1234 & 1234 \\
\hline Safety and heal th & 1234 & 1234 & 1234 & 1234 & 1234 \\
\hline Transportation issues & 1234 & 1234 & 1234 & 1234 & 1234 \\
\hline Packaging issues & 1234 & 1234 & 1234 & 1234 & 1234 \\
\hline
\end{tabular}


PART 2 - IDEAL SKILLS FOR EACH TASK

\begin{tabular}{|c|c|c|c|c|c|}
\hline$\frac{\text { IASKS } \cdots>}{\text { SKILLS/KHOULEDCE }}$ & $\begin{array}{c}\text { PROVIDE AMALYSES TO } \\
\text { DETERMIHE EFFECTS } \\
\text { OF REKEDIATIOW } \\
\text { PROAIECIS } \\
\end{array}$ & $\begin{array}{c}\text { REVIEU REMEDIATIIOW } \\
\text { PROJECTS AND THEIR } \\
\text { PROGRESS } \\
\end{array}$ & $\begin{array}{c}\text { RECOWENWO ACTIOWS } \\
\text { TO CORRECT REWEDIAL } \\
\text { PROOULEMS }\end{array}$ & $\begin{array}{c}\text { IOEMTIFY AND } \\
\text { DOCANEWI } \\
\text { DEFICIENCIES } \\
\end{array}$ & SET SCHEOULES \\
\hline biology, zoology, and ecology & 1234 & 1234 & 1234 & 1234 & 1234 \\
\hline Chemistry & 1234 & 1234 & 1234 & 1234 & 1234 \\
\hline Nuclear engincering & 1234 & 1234 & 1234 & 1234 & 1234 \\
\hline Physicol sciences & 1234 & 1234 & 1234 & 1234 & 1234 \\
\hline Geohydrology & 1234 & 1234 & 1234 & 1234 & 1234 \\
\hline Cartography & 1234 & 1234 & 1234 & 1234 & 1234 \\
\hline Geophysics & 1234 & 1234 & 1234 & 1234 & 1234 \\
\hline D8D techniques & 1234 & 1234 & 1234 & 1234 & 1234 \\
\hline Cultural diversity issues & 1234 & 1234 & 1234 & 1234 & 1234 \\
\hline $\begin{array}{l}\text { Hazardous, chemical, radioactive } \\
\text { and mixed waste management }\end{array}$ & $123 \quad 4$ & 1234 & 1234 & 1234 & 1234 \\
\hline RCRA/CERCLA process & 1234 & 1234 & 1234 & 1234 & 1234 \\
\hline Natural resources & 1234 & 1234 & 1234 & 1234 & 1234 \\
\hline Land use & 1234 & 1234 & 1234 & 1234 & 1234 \\
\hline Hildlife management & 1234 & 12341 & 1234 & 1234 & 1234 \\
\hline Soils, and minerals & 1234 & 1234 & 1234 & 1234 & 1234 \\
\hline Aquatic resources & 1234 & 1234 & 1234 & 1234 & 1234 \\
\hline NEPA process & 1234 & 1234 & 1234 & 1234 & 1234 \\
\hline Environmental sciences & 1234 & 1234 & 1234 & 1234 & 1234 \\
\hline Quality assurance & 1234 & 1234 & 1234 & 1234 & 1234 \\
\hline Emergency response procedures & 1234 & 1234 & 1234 & 1234 & 1234 \\
\hline
\end{tabular}


PART 2 - IDEAL SKILLS FOR EACH TASK

\begin{tabular}{|c|c|c|c|c|c|}
\hline $\begin{array}{c}\text { TASKS }-\cdots> \\
\text { SXILLS/KNOAREOCE }\end{array}$ & $\begin{array}{l}\text { IDEHTIFY \& COWOUCT } \\
\text { FACILITY ACCEPTAHCE }\end{array}$ & $\begin{array}{l}\text { OVERSEE ASSESSHEWIS } \\
\text { FOR CLEAM UP (E.G.. } \\
\text { RISK, FEASIBILITY, } \\
\text { UIER, QUULITY. } \\
\text { EIC.) }\end{array}$ & $\begin{array}{c}\text { OVERSEE } \\
\text { ENVIROGWENTAL } \\
\text { RESTORATION } \\
\text { ACTIVITIES (CERCLA) } \\
\end{array}$ & $\begin{array}{c}\text { ONERSEE UASTE } \\
\text { MULAGEMENT } \\
\text { ACTIVITIIES (RCRA) } \\
\end{array}$ & $\begin{array}{l}\text { OVERSEE TOXIC \& } \\
\text { HUZARDOWS MAIERIALS } \\
\text { MUMAGEHEWI } \\
\text { ACIIVIIIES } \\
\end{array}$ \\
\hline National consensus standards & 1234 & $123 \quad 4$ & 1234 & 1234 & 1234 \\
\hline $\begin{array}{l}\text { Interogency agreements, } \\
\text { adninistration, ond process }\end{array}$ & 1234 & 1234 & 1234 & 1234 & 1234 \\
\hline $\begin{array}{l}\text { organizing information and } \\
\text { present cosent arguments }\end{array}$ & 1234 & 1234 & 1234 & 1234 & 1234 \\
\hline Writing succinctly and clearly & 1234 & 1234 & 1234 & 1234 & 1234 \\
\hline $\begin{array}{l}\text { Public speaking (e.9., public, } \\
\text { medio, other employees and } \\
\text { speciat interest groups) }\end{array}$ & 1234 & 1234 & 1234 & 1234 & 1234 \\
\hline Negotiating/conflict resolution & 1234 & 1234 & 1234 & 1234 & 1234 \\
\hline $\begin{array}{l}\text { Providing clear guidance ond } \\
\text { direction to accomplish tasks }\end{array}$ & 1234 & 1234 & 1234 & 1234 & 1234 \\
\hline Meeting facilitation/leadership & 1234 & 1234 & 1234 & 1234 & 1234 \\
\hline DOE managernent strategies & 1234 & 1234 & 1234 & 1234 & 1234 \\
\hline $\begin{array}{l}\text { EH's mission, policies, } \\
\text { management pians, procedures, } \\
\text { priorities, gools and } \\
\text { objectives }\end{array}$ & 1234 & 1234 & 1234 & 1234 & 1234 \\
\hline DOE departmental organization & 1234 & 1234 & 1234 & 1234 & 1234 \\
\hline $\begin{array}{l}\text { Contractor organization and } \\
\text { procedures }\end{array}$ & 1234 & 1234 & 1234 & 1234 & 1234 \\
\hline Decision analysis & 1234 & 1234 & 1234 & 1234 & 1234 \\
\hline Conducting library research & 1234 & 1234 & 1234 & 1234 & 1234 \\
\hline $\begin{array}{l}\text { Facility, site, operations and } \\
\text { processes }\end{array}$ & $\begin{array}{llll}1 & 2 & 3 & 4\end{array}$ & 1234 & 1234 & 1234 & 1234 \\
\hline $\begin{array}{l}\text { Defining boundaries of a } \\
\text { problenn }\end{array}$ & 1234 & 1234 & 1234 & 1234 & 1234 \\
\hline $\begin{array}{l}\text { Keeping abreast of current } \\
\text { developments }\end{array}$ & 1234 & $\begin{array}{llll}12 & 3 & 4\end{array}$ & 1234 & 1234 & 1234 \\
\hline Organizing/categorizing & 1234 & 1234 & $\begin{array}{llll}1 & 2 & 3 & 4\end{array}$ & 1234 & 1234 \\
\hline
\end{tabular}

$6 / 16 / 93$ 
PART 2 - IDEAL SKILLS FOR EACH TASK

\begin{tabular}{|c|c|c|c|c|c|}
\hline $\begin{array}{c}\text { TASKS } \cdots> \\
\text { SKILLS/KHOMEDECE } \\
\end{array}$ & $\begin{array}{l}\text { IOEHYIFY CONOUCT } \\
\text { FACILIIY ACCEPIANCE } \\
\end{array}$ & $\begin{array}{l}\text { OVERSEE ASSESSWENTS } \\
\text { FOR CLEAN UP (E.G.., } \\
\text { RISK, FEASIBILITY, } \\
\text { UAIER, QULIIYY. } \\
\text { EYC. }\end{array}$ & $\begin{array}{c}\text { OVERSEE } \\
\text { EHVIROWHEMTAL } \\
\text { RESTORATIOA } \\
\text { ACTIVIIIES (CERCLA) } \\
\end{array}$ & $\begin{array}{c}\text { OVERSEE WUSIE } \\
\text { MUMACEMENT } \\
\text { ACTIVITIES (RCRA) } \\
\end{array}$ & $\begin{array}{c}\text { OVERSEE YOXIC } 2 \\
\text { MUZZROOUS MAIERIALS } \\
\text { MAMACEHEMI } \\
\text { ACIIVIIIIS } \\
\end{array}$ \\
\hline $\begin{array}{l}\text { Operating equipment (e.g., } \\
\text { test ing equipnent, respirators) }\end{array}$ & 1234 & 1234 & 1234 & 1234 & 1234 \\
\hline Operating and using computer & 1234 & 1234 & 1234 & 1234 & 1234 \\
\hline louch typing & 1234 & 1234 & 1234 & 1234 & 1234 \\
\hline $\begin{array}{l}\text { Understanding principles of } \\
\text { data buse requirenents und } \\
\text { laset ine techniques }\end{array}$ & 1234 & 1234 & 1234 & 1234 & 1234 \\
\hline $\begin{array}{l}\text { Principles of DOE budget } \\
\text { formulations, budget execution } \\
\text { and cost account ing, and the } \\
\text { procedures involved }\end{array}$ & 1234 & 1234 & 1234 & 1234 & 1234 \\
\hline Schedule constraints & 1234 & 1234 & 1234 & 1234 & 1234 \\
\hline $\begin{array}{l}\text { Planning, programing, funding } \\
\text { and acquisition management }\end{array}$ & $\begin{array}{llll}1 & 2 & 3 & 4\end{array}$ & 1234 & 1234 & 1234 & 1234 \\
\hline $\begin{array}{l}\text { Conducting analyses involving } \\
\text { camplex variables }\end{array}$ & 1234 & 1234 & $\begin{array}{llll}1 & 2 & 3 & 4\end{array}$ & 1234 & 1234 \\
\hline Supervising staff/contractors & 1234 & 1234 & 1234 & 1234 & 1234 \\
\hline $\begin{array}{l}\text { Delegating authority and } \\
\text { responsibility }\end{array}$ & $\begin{array}{llll}1 & 2 & 3 & 4\end{array}$ & $\begin{array}{lllll}1 & 2 & 3 & 4\end{array}$ & 1234 & 1234 & 1234 \\
\hline $\begin{array}{l}\text { Equal Opportunity programs } \\
\text { plans }\end{array}$ & 1234 & 1234 & 1234 & 1234 & 9234 \\
\hline $\begin{array}{l}\text { Analyzing/synthesizing } \\
\text { information }\end{array}$ & 1234 & 1234 & 1234 & 1234 & 1234 \\
\hline $\begin{array}{l}\text { Assessing strengths and } \\
\text { Heaknesses of alternatives }\end{array}$ & 1234 & 1234 & 1234 & 1234 & 1234 \\
\hline $\begin{array}{l}\text { Anticipating unintended } \\
\text { consequences }\end{array}$ & 1234 & 1234 & 1234 & 1234 & 1234 \\
\hline $\begin{array}{l}\text { Comparing existing conditions } \\
\text { to pre-set standards }\end{array}$ & 1234 & 1234 & 1234 & 1234 & 1234 \\
\hline Prioritizing based on criteria & $123 \cdot 4$ & 1234 & 1234 & 1234 & 1234 \\
\hline $\begin{array}{l}\text { Identifying relevant } \\
\text { material/requirements }\end{array}$ & $\begin{array}{llll}1 & 2 & 3 & 4\end{array}$ & $\begin{array}{llll}1 & 2 & 3 & 4\end{array}$ & $\begin{array}{llll}1 & 2 & 3 & 4\end{array}$ & $\begin{array}{llll}12 & 2 & 4\end{array}$ & 1234 \\
\hline
\end{tabular}


PART 2 - IDEAL SKILLS FOR EACH TASK

\begin{tabular}{|c|c|c|c|c|c|}
\hline $\begin{array}{c}\text { IASKS } \cdots>\text { - } \\
\text { SKILLS/KHOLLEOCE }\end{array}$ & $\begin{array}{l}\text { IDENTIFY \& COWOUCT } \\
\text { FACILIIY ACCEPTANCE } \\
\end{array}$ & $\begin{array}{l}\text { OVERSEE ASSESSWEMTS } \\
\text { FOR CLEAM UP (E.G.., } \\
\text { RISK, FEASIBILIIIY, } \\
\text { UAIER, QULIIY, } \\
\text { EIC.) }\end{array}$ & $\begin{array}{c}\text { OVERSEE } \\
\text { ENYIROWHENTAL } \\
\text { RESIORAYIOW } \\
\text { ACTIVIIIES (CERCLA) } \\
\end{array}$ & $\begin{array}{c}\text { OVERSEE UUSTE } \\
\text { MUKACEMENT } \\
\text { ACTIVIIILS (RCRA) } \\
\end{array}$ & 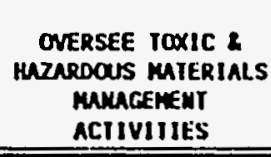 \\
\hline Delegating tasks & 1234 & 1234 & 1234 & 1234 & 1234 \\
\hline Defining upplicable criteria & 1234 & 1234 & 1234 & 1234 & 1234 \\
\hline $\begin{array}{l}\text { Reading technical material for } \\
\text { a set purpose }\end{array}$ & 1234 & 1234 & 1234 & 1234 & 1234 \\
\hline Speed reuding & 1234 & 1234 & 1234 & 1234 & 1234 \\
\hline Consol idating naterials & 1234 & 1234 & 1234 & 1234 & 1234 \\
\hline $\begin{array}{l}\text { Examining trade-offs among } \\
\text { alternatives }\end{array}$ & 1234 & 1234 & 1234 & 1234 & 1234 \\
\hline $\begin{array}{l}\text { Geonapping, remore sensing, and } \\
\text { characterization techniques }\end{array}$ & 1234 & 1234 & 1234 & 1234 & 1234 \\
\hline $\begin{array}{l}\text { Proper engineering/technical } \\
\text { standards }\end{array}$ & 1234 & 1234 & 1234 & 1234 & 1234 \\
\hline Valid engineering solutions & 1234 & 1234 & 1234 & 1234 & 1234 \\
\hline Remediation techniques & 1234 & 1234 & 1234 & 1234 & 1234 \\
\hline $\begin{array}{l}\text { Uaste treatment/disposal } \\
\text { technologies }\end{array}$ & $\begin{array}{llll}12 & 2 & 4 \\
\end{array}$ & $1234^{\prime}$ & 1234 & 1234 & 1234 \\
\hline Hunan heel th impacts & 1234 & 1234 & 1234 & 1234 & 1234 \\
\hline Radiation effects & $\begin{array}{llll}1 & 2 & 3 & 4 \\
\end{array}$ & 1234 & 1234 & 1234 & 1234 \\
\hline $\begin{array}{l}\text { Heal th and radiological safety } \\
\text { impacts and benefits }\end{array}$ & 1234 & 1234 & 1234 & 1234 & 1234 \\
\hline Heal th physics & $123 \quad 3 \quad 4$ & 1234 & 1234 & 1234 & 1234 \\
\hline Safety and health & 1234 & 1234 & 1234 & 1234 & 1234 \\
\hline Safety and health & 1234 & 1234 & $123 \quad 4$ & 1234 & 1234 \\
\hline Ironsportotion issues & 1234 & 1234 & 1234 & 1234 & 1234 \\
\hline Packoging issues & 1234 & 1234 & 1234 & 1234 & 1234 \\
\hline Biology, zoology, and ecology & 1234 & 1234 & 1234 & 1234 & 1234 \\
\hline Chenistry & 1234 & 1234 & 1234 & 1234 & 1234 \\
\hline
\end{tabular}


PART 2 - IDEAL SKILLS FOR EACH TASK

\begin{tabular}{|c|c|c|c|c|c|}
\hline $\begin{array}{r}\text { rASKS }-\cdots> \\
\text { SKILLS/KHOAREDGE } \\
\end{array}$ & $\begin{array}{l}\text { IDENTIFY \& COWOUCT } \\
\text { FACILIIY ACCEPTAKCE }\end{array}$ & $\begin{array}{l}\text { OVERSEE ASSESSHEWIS } \\
\text { FOR CLEAN UP (E.G.., } \\
\text { RISK, FEASIBILITY, } \\
\text { UAIER, QUALITY, } \\
\text { EIC) }\end{array}$ & $\begin{array}{c}\text { OVERSEE } \\
\text { EMVIROAWEMTAL. } \\
\text { RESTORAIION } \\
\text { ACTIVITIES (CERCLA) } \\
\end{array}$ & $\begin{array}{c}\text { OVERSEE UASIE } \\
\text { MULACEMENI } \\
\text { ACTIVITIES (RCRA) } \\
\end{array}$ & $\begin{array}{l}\text { OVERSEE TOXIC } \\
\text { HAZARDOUS MAIERIALS } \\
\text { MUACENENI } \\
\text { ACTIVIIIES }\end{array}$ \\
\hline Nuclear engineering & 1234 & 1234 & 1234 & 1234 & 1234 \\
\hline Physical sciences & 1234 & 1234 & 1234 & 1234 & 1234 \\
\hline Geohydrology & 1234 & 1234 & 1234 & 1234 & 1234 \\
\hline Cartography & 1234 & 1234 & 1234 & 1234 & 1234 \\
\hline Ceophysics & 1234 & 1234 & 1234 & 1234 & 1234 \\
\hline D80 techniques & 1234 & 1234 & 1234 & 1234 & 1234 \\
\hline Cul tural diversity issues & 1234 & 1234 & 1234 & 1234 & 1234 \\
\hline $\begin{array}{l}\text { Hazardaus, chemical, } \\
\text { radioactive and mixed waste } \\
\text { management }\end{array}$ & 1234 & 1234 & 1234 & $123 \quad 4$ & 1234 \\
\hline RCRA/CERCLA process & 1234 & $123 \quad 3 \quad 4$ & 1234 & 1234 & 1234 \\
\hline Natural resources & 1234 & 1234 & 1234 & 1234 & 1234 \\
\hline Land use & 1234 & 1234 & 1234 & 1234 & 1234 \\
\hline Hildl ife management & 1234 & 1234 & 1234 & 1234 & 1234 \\
\hline Soils, and minerals & 1234 & 123.4 & 1234 & 1234 & 1234 \\
\hline Aquatic resources & 1234 & $123 \quad 3$ & 1234 & 1234 & 1234 \\
\hline NEPA process & 1234 & 1234 & 1234 & 1234 & 1234 \\
\hline Environmental sciences & 1234 & 1234 & 1234 & $123 \quad 4$ & 1234 \\
\hline Quality assurance & 1234 & 1234 & 1234 & 1234 & 1234 \\
\hline Emergency response procedures & 1234 & 1234 & 1234 & 1234 & 1234 \\
\hline
\end{tabular}


PART 2 - IDEAL SKILLS FOR EACH TASK

\begin{tabular}{|c|c|c|c|c|c|c|}
\hline $\begin{array}{r}\text { YASKS } \cdots \text {-.-> } \\
\text { SKILLS/KWOULEDCE } \\
\end{array}$ & $\begin{array}{c}\text { OVERSEE UATER } \\
\text { cunLIIY ACIIVITIES } \\
\end{array}$ & $\begin{array}{c}\text { OVERSEE AIR QULITY } \\
\text { ACIIVIIIES } \\
\end{array}$ & $\begin{array}{c}\text { OVERSEE CULTURAL } \\
\text { RESOURCES MURAEETEMT } \\
\text { ACTIVITIES } \\
\end{array}$ & $\begin{array}{c}\text { OVERSEE RNDIATIOW } \\
\text { PROTECTION } \\
\text { ACIIVITIES } \\
\end{array}$ & $\begin{array}{c}\text { ONERSEE MEPA } \\
\text { ACTIVITIES }\end{array}$ & \\
\hline National consensus stundards & 1234 & 1234 & 1234 & 1234 & 1234 & \\
\hline $\begin{array}{l}\text { Interagency agreements, } \\
\text { udininistration, and process }\end{array}$ & 1234 & 1234 & 1234 & 1234 & 1234 & 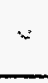 \\
\hline $\begin{array}{l}\text { Organizing information and } \\
\text { present cogent orgunents }\end{array}$ & 1234 & 1234 & 1234 & 1234 & 1234 & \\
\hline Writing succinctly and clearly & 1234 & 1234 & 1234 & 1234 & 1234 & \\
\hline $\begin{array}{l}\text { Public speaking (e.9.. public } \\
\text { presentations, employee/employer } \\
\text { interactions) }\end{array}$ & 1234 & 1234 & 1234 & 1234 & 1234 & - \\
\hline $\begin{array}{l}\text { Interpersonal facility (e.9., } \\
\text { public, media, other employees } \\
\text { and special interest groups) }\end{array}$ & 1234 & 1234 & 1234 & 1234 & 1234 & \\
\hline Hegotiating/conflict resolution & 1234 & 1234 & 1234 & 1234 & 1234 & \\
\hline $\begin{array}{l}\text { Providing clear guidance and } \\
\text { direction to acconpl ish tasks }\end{array}$ & 1234 & 1234 & 1234 & 1234 & 1234 & \\
\hline Meeting facilitation/leadership & 1234 & 1234 & 1234 & 1234 & 1234 & \\
\hline DOE management strotegies & 1234 & 1234 & 1234 & 1234 & 1234 & \\
\hline $\begin{array}{l}\text { EM's mission, policies, } \\
\text { inanagement plans, procedures, } \\
\text { priorities, gools and objectives }\end{array}$ & 1234 & $\begin{array}{r}1234 \\
123 \\
\end{array}$ & 1234 & 1234 & $123 \quad 3$ & \\
\hline Doe departmental organization & 1234 & 1234 & 1234 & 1234 & 1234 & \\
\hline $\begin{array}{l}\text { Contractor organization and } \\
\text { procedures }\end{array}$ & 1234 & 1234 & 1234 & 1234 & 1234 & \\
\hline Decision analysis & 1234 & 1234 & 1234 & 1234 & 1234 & \\
\hline Conducting library research & 1234 & 1234 & 1234 & 1234 & 1234 & \\
\hline $\begin{array}{l}\text { Facility, site, operations and } \\
\text { processes }\end{array}$ & 1234 & 1234 & 1234 & 1234 & 1234 & \\
\hline Defining boundaries of a problem & 1234 & 1234 & 1234 & 1234 & 1234 & \\
\hline $\begin{array}{l}\text { Keeping abreast of current } \\
\text { developments }\end{array}$ & 1234 & 1234 & 1234 & 1234 & 1234 & \\
\hline Organizing/categorizing & 1234 & 1234 & 1234 & 1234 & 1234 & \\
\hline
\end{tabular}


PART 2 - IDEAL SKILLS FOR EACH TASK

\begin{tabular}{|c|c|c|c|c|c|}
\hline $\begin{array}{c}\text { IASKS } \cdots \text { - - > } \\
\text { SKILLS/KMOAREDCE }\end{array}$ & $\begin{array}{l}\text { OVERSEE WATER } \\
\text { QULIIY ACTIVITIES } \\
\end{array}$ & $\begin{array}{l}\text { OVERSEE AIR quALITY } \\
\text { ACTIVITIES }\end{array}$ & $\begin{array}{c}\text { OVERSEE OULIURAL } \\
\text { RESOURCES MMNAGEMEHT } \\
\text { ACTIVITIES }\end{array}$ & $\begin{array}{l}\text { OVERSEE RRDIATION } \\
\text { PROTECTIOW } \\
\text { ACIIVITIES } \\
\end{array}$ & $\begin{array}{l}\text { OVERSEE MEPA } \\
\text { ACIIVIYIES } \\
\end{array}$ \\
\hline $\begin{array}{l}\text { Operating equipnent (e.g., } \\
\text { test ing tquiputent, respi rutors) }\end{array}$ & 1234 & 1234 & 1234 & 1234 & 1234 \\
\hline Operating and using conputer & 1234 & 1234 & 1234 & 1234 & 1234 \\
\hline Touch typing & 1234 & 1234 & 1234 & 1234 & 1234 \\
\hline $\begin{array}{l}\text { Understanding principles of data } \\
\text { base requirentents and basel ine } \\
\text { techniques }\end{array}$ & 1234 & 1234 & 1234 & 1234 & 1234 \\
\hline $\begin{array}{l}\text { Using software and applying it to } \\
\text { tasks }\end{array}$ & 1234 & 1234 & 1234 & 1234 & 1234 \\
\hline Time nanagement & 1234 & 1234 & 1234 & 1234 & 1234 \\
\hline Heighing costs versus benefits & 1234 & 1234 & 1234 & 1234 & 1234 \\
\hline $\begin{array}{l}\text { Project or program management } \\
\text { techniques }\end{array}$ & 1234 & 1234 & 1234 & 1234 & 1234 \\
\hline $\begin{array}{l}\text { Principles of DOE budget } \\
\text { formulations, budget execution } \\
\text { and cost accounting, and the } \\
\text { procedures involved }\end{array}$ & 1234 & 1234 & 1234 & 1234 & 1234 \\
\hline Schedule constraints & 1234 & 1234 & 1234 & 1234 & 1234 \\
\hline $\begin{array}{l}\text { Planning, programning, funding } \\
\text { and acquisition managentent }\end{array}$ & 1234 & 1234 & 1234 & 1234 & 1234 \\
\hline $\begin{array}{l}\text { Conducting analyses involving } \\
\text { complex variables }\end{array}$ & 1234 & 1234 & 1234 & 1234 & 1234 \\
\hline Supervising staff/contractors & 1234 & 1234 & 1234 & 1234 & 1234 \\
\hline $\begin{array}{l}\text { Delegating authority and } \\
\text { responsibility }\end{array}$ & 123.4 & 123.4 & 1234 & 1234 & 1234 \\
\hline Equal Opportunity program plans & 1234 & 1234 & 1234 & 1234 & 1234 \\
\hline $\begin{array}{l}\text { Analyzing/synthesizing } \\
\text { information }\end{array}$ & 1234 & 1234 & 1234 & 1234 & 1234 \\
\hline $\begin{array}{l}\text { Assessing strengths and } \\
\text { weaknesses of alternatives }\end{array}$ & 1234 & 1234 & 1234 & 1234 & 1234 \\
\hline $\begin{array}{l}\text { Anticipating unintended } \\
\text { consequences }\end{array}$ & $\begin{array}{llll}1 & 2 & 3 & 4\end{array}$ & $\begin{array}{llll}1 & 2 & 3 & 4\end{array}$ & 12334 & 1234 & 1234 \\
\hline
\end{tabular}


PART 2 - IDEAL SKILLS FOR EACH TASK

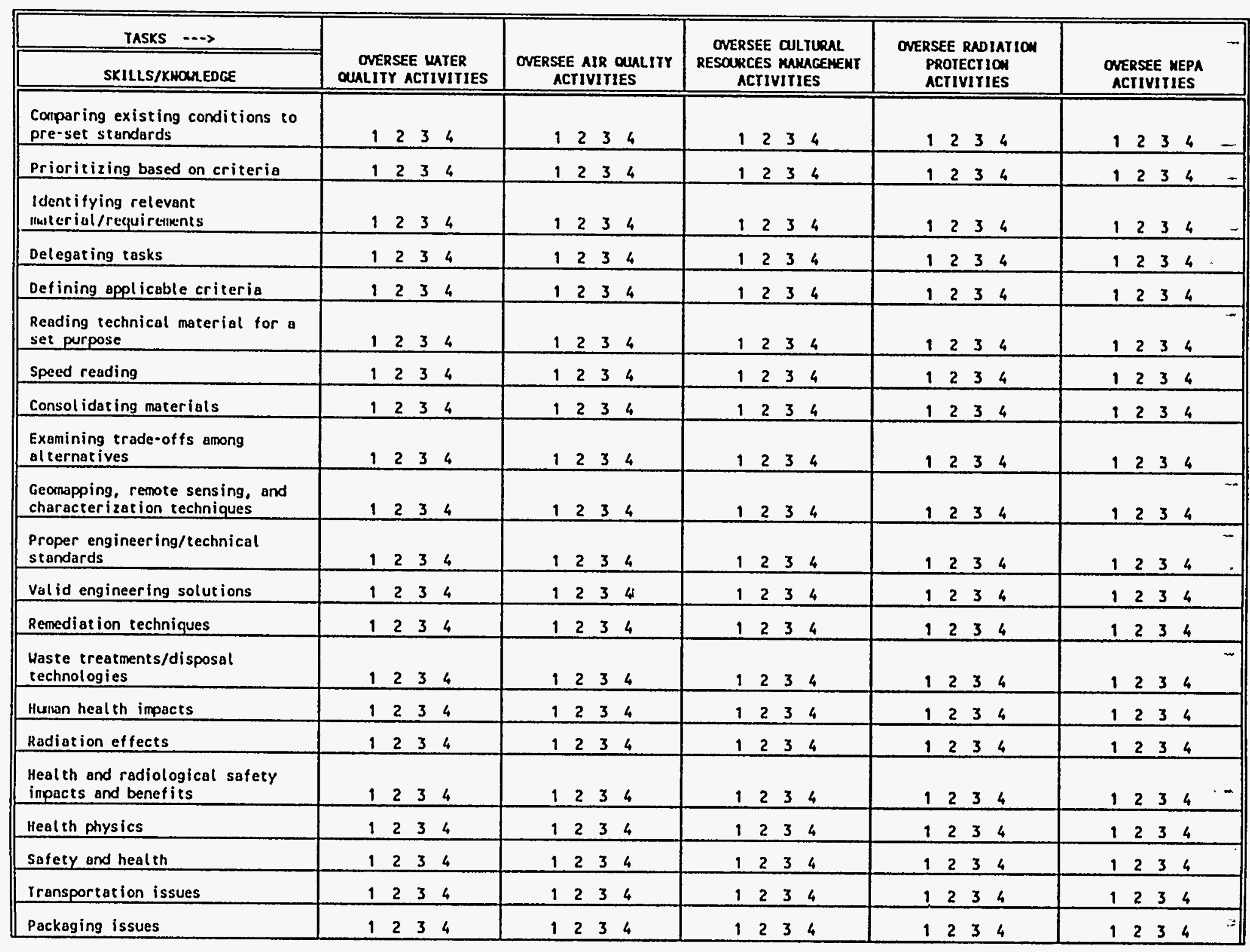


PART 2 - IDEAL SKILLS FOR EACH TASK

\begin{tabular}{|c|c|c|c|c|c|}
\hline TASKS $\cdots$ - & $\begin{array}{c}\text { OVERSEE MATER } \\
\text { OULLITY ACIIVITIES } \\
\end{array}$ & $\begin{array}{c}\text { OVERSEE AIR QLALITY } \\
\text { ACTIVITIES } \\
\end{array}$ & $\begin{array}{c}\text { OVERSEE OLITURAL } \\
\text { RESOURCES MUAGEKENT } \\
\text { ACIIVITIES } \\
\end{array}$ & $\begin{array}{c}\text { OVERSEE RADIATION } \\
\text { PROIECIIOW } \\
\text { ACTIVITIES } \\
\end{array}$ & $\begin{array}{c}\text { OWERSEE MEPA } \\
\text { ACIIVITIES }\end{array}$ \\
\hline Biology, zoology, and ecology & 1234 & 1234 & 1234 & 1234 & 1234 \\
\hline Chemistry & 1234 & $123 \quad 3$ & 1234 & 1234 & 1234 \\
\hline Huclear engineering & $123 \quad 3 \quad 4$ & 1234 & 1234 & 1234 & 1234 \\
\hline Physical sciences & 1234 & 1234 & 1234 & 1234 & 1234 \\
\hline Geohydrology & $123 \quad 3 \quad 4$ & 1234 & 1234 & 1234 & 1234 \\
\hline Cartography & 1234 & 1234 & 1234 & 1234 & 1234 \\
\hline Geophysics & 1234 & 1234 & 1234 & 1234 & 1234 \\
\hline D80 techniques & 1234 & 1234 & $123 \quad 4$ & 1234 & 1234 \\
\hline Cultural diversity issues & 1234 & 1234 & 1234 & 1234 & 1234 \\
\hline $\begin{array}{l}\text { Hazardous, chemical, radioactive } \\
\text { and mixed waste management }\end{array}$ & 1234 & 1234 & 1234 & 1234 & 1234 \\
\hline RCRA/CERCLA process & 1234 & 1234 & 1234 & 1234 & 1234 \\
\hline Natural resources & 1234 & 1234 & 1234 & 1234 & 1234 \\
\hline Land use & $123 \quad 3 \quad 4$ & 1234 & 1234 & 1234 & 1234 \\
\hline Wildlife management & 1234 & 123.4 & 1234 & 1234 & 1234 \\
\hline Soils, and minerals & 1234 & 1234 & 1234 & 1234 & 1234 \\
\hline Aguatic resources & 1234 & 1234 & 1234 & 1234 & 1234 \\
\hline NEPA process & 1234 & 1234 & 1234 & 1234 & 1234 \\
\hline Environmental sciences & 1234 & 1234 & 1234 & 1234 & 1234 \\
\hline Quality assurance & 1234 & 1234 & 1234 & 1234 & 1234 \\
\hline Emergency response procedures & 1234 & 1234 & 1234 & 1234 & 1234 \\
\hline
\end{tabular}


PART 2 - IDEAL SKILLS FOR EACH TASK

\begin{tabular}{|c|c|c|c|c|c|}
\hline $\begin{array}{c}\text { TASKS }-\cdots \\
\text { SKILLS/KKOUREDCE } \\
\end{array}$ & $\begin{array}{c}\text { OVERSEE } \\
\text { DECONINHIMAIIOH } \\
\text { DECOAMISSIONIHG } \\
\end{array}$ & $\begin{array}{l}\text { OVERSEE QULITY } \\
\text { ASSURAKCE/OUALITY } \\
\text { COHIROL ACTIVITIES } \\
\end{array}$ & $\begin{array}{c}\text { OVERSEE LAN USE } \\
\text { ACIIVITIES } \\
\end{array}$ & $\begin{array}{c}\text { EVALUAIE } \\
\text { ALIERMAIIVES FOR } \\
\text { CLENUP } \\
\end{array}$ & $\begin{array}{l}\text { OVERSEE PROCRAM } \\
\text { EVALUIIIONS } \\
\end{array}$ \\
\hline National consensus standards & 1234 & 1234 & 1234 & 1234 & 1234 \\
\hline $\begin{array}{l}\text { Interagency agreements, } \\
\text { autministration, and process }\end{array}$ & 1234 & 1234 & 1234 & 1234 & 1234 \\
\hline $\begin{array}{l}\text { Organizing information and } \\
\text { present cogent orguments }\end{array}$ & $123 \quad 4$ & 1234 & 1234 & 1234 & 1234 \\
\hline Writing succinctly and clearly & 1234 & 1234 & 1234 & 1234 & 1234 \\
\hline $\begin{array}{l}\text { Publ ic speuking (e.9., public } \\
\text { presentutions, etrployee/ empl oyer } \\
\text { interactions) }\end{array}$ & 1234 & 1234 & 1234 & 1234 & 1234 \\
\hline Hegotiating/conflict resolution & 1234 & 1234 & 1234 & 1234 & 1234 \\
\hline $\begin{array}{l}\text { Providing clear guidance and } \\
\text { direction to accomplish tasks }\end{array}$ & 1234 & 1234 & 1234 & 1234 & 1234 \\
\hline Meeting facilitotion/leadership & 1234 & 1234 & 1234 & 1234 & 1234 \\
\hline DOE management strategies & 1234 & 1234 & 1234 & 1234 & 1234 \\
\hline $\begin{array}{l}\text { EH's mission, policies, } \\
\text { management plans, procedures, } \\
\text { priorities, goals and objectives }\end{array}$ & 1234 & 1234 & 1234 & 1234 & 1234 \\
\hline DOE departmental organization & 1234 & $123 \quad 4$ & 1234 & 1234 & 1234 \\
\hline $\begin{array}{l}\text { Contractor organization and } \\
\text { procedures }\end{array}$ & 1234 & 1234 & 1234 & 1234 & 1234 \\
\hline Decision analysis & 1234 & 1234 & 1234 & 1234 & 1234 \\
\hline Conducting library research & 1234 & 1234 & 1234 & 1234 & 1234 \\
\hline $\begin{array}{l}\text { Facility, site, operations and } \\
\text { processes }\end{array}$ & 1234 & 1234 & $123 \quad 4$ & 1234 & 1234 \\
\hline Defining boundaries of a problem & $123 \quad 34$ & 1234 & 1234 & 1234 & 1234 \\
\hline $\begin{array}{l}\text { Keeping abreast of current } \\
\text { developments }\end{array}$ & 1234 & 1234 & 1234 & 1234 & 1234 \\
\hline Organizing/categorizing & 1234 & 1234 & 1234 & 1234 & 1234 \\
\hline $\begin{array}{l}\text { Operating equipment (e.g.. } \\
\text { testing equipment, respirators) }\end{array}$ & 1234 & 1234 & 1234 & 1234 & 1234 \\
\hline Operating and using computer & 1234 & 1234 & 1234 & 1234 & 1234 \\
\hline
\end{tabular}

$6 / 16 / 93$

31 
PART 2 - IDEAL SKILLS FOR EACH TASK

\begin{tabular}{|c|c|c|c|c|c|}
\hline $\begin{array}{c}\text { IASKS } \cdots \text {--.> } \\
\text { SKILLS/KHOULEDCE }\end{array}$ & $\begin{array}{c}\text { OVERSEE } \\
\text { DECOMTAMIMATION } 2 \\
\text { DECOMWISSIOWIHG }\end{array}$ & $\begin{array}{l}\text { ONERSEE QUNLITY } \\
\text { ASSURANCE/QWALITY } \\
\text { COWTROL ACTIVITIES }\end{array}$ & $\begin{array}{l}\text { OVERSEE LAND USE } \\
\text { ACIIVITIES }\end{array}$ & $\begin{array}{c}\text { EVALUAIE } \\
\text { ALTERHATIVES FOR } \\
\text { CLEAMUP } \\
\end{array}$ & $\begin{array}{l}\text { OVERSEE PROCRAM } \\
\text { EVALLUIIOWS }\end{array}$ \\
\hline Touch typing & 1234 & 1234 & 1234 & $123 \quad 4$ & 1234 \\
\hline $\begin{array}{l}\text { Understanding principles of data } \\
\text { base requirements and baseline } \\
\text { ecchniques }\end{array}$ & 1234 & 1234 & 1234 & 1234 & 1234 \\
\hline $\begin{array}{l}\text { Using sof tware and applying it } \\
\text { to tasks }\end{array}$ & 1234 & 1234 & 1234 & 1234 & 1234 \\
\hline rigre management & 1234 & 1234 & 1234 & 1234 & 1234 \\
\hline Heighing costs versus benefits & 1234 & 1234 & 1234 & 1234 & 1234 \\
\hline $\begin{array}{l}\text { Project or program management } \\
\text { techniques }\end{array}$ & 1234 & 1234 & 1234 & 1234 & 1234 \\
\hline $\begin{array}{l}\text { Principles of DoE budget } \\
\text { fornulations, budget execution } \\
\text { and cost account ing, and the } \\
\text { procedures involved }\end{array}$ & 1234 & 1234 & 1234 & 1234 & 1234 \\
\hline Schedule constraints & 1234 & 1234 & 1234 & 1234 & 1234 \\
\hline $\begin{array}{l}\text { Planning, programing, funding } \\
\text { and acquisition management }\end{array}$ & 1234 & 1234 & 1234 & 1234 & 1234 \\
\hline $\begin{array}{l}\text { Conducting analyses involving } \\
\text { complex variables }\end{array}$ & 1234 & 1234 & 1234 & 1234 & 1234 \\
\hline Supervising staff/contractors & 1234 & 1234 & 1234 & 1234 & 1234 \\
\hline $\begin{array}{l}\text { Delegat ing authority and } \\
\text { responsibitity }\end{array}$ & 1234 & 1234 & 1234 & 1234 & 1234 \\
\hline Equal Opportunity program plans & 1234 & 1234 & 1234 & 1234 & $\begin{array}{llll}1 & 2 & 3 & 4\end{array}$ \\
\hline $\begin{array}{l}\text { Analyzing/synthesizing } \\
\text { information }\end{array}$ & 1234 & 1234 & $123-4$ & 1234 & 1234 \\
\hline $\begin{array}{l}\text { Assessing strengths and } \\
\text { Neaknesses of alternatives }\end{array}$ & 1234 & 1234 & 1234 & 1234 & 1234 \\
\hline $\begin{array}{l}\text { Anticipating unintended } \\
\text { consequences }\end{array}$ & 1234 & 1234 & 1234 & 1234 & 1234 \\
\hline $\begin{array}{l}\text { Comparing existing conditions to } \\
\text { pre-set standards }\end{array}$ & 1234 & 1234 & $123 \quad 3 \quad 4$ & 1234 & 1234 \\
\hline Prioritizing based on criteria & 1234 & 1234 & 12334 & 1234 & 1234 \\
\hline
\end{tabular}


PART 2 - IDEAL SKILLS FOR EACH TASK

\begin{tabular}{|c|c|c|c|c|c|}
\hline IASXS $-\cdots$ - & $\begin{array}{c}\text { OVERSEE } \\
\text { DECONIAMIMAIIOH } 2 \\
\text { DECOMAIISSIOWING }\end{array}$ & $\begin{array}{l}\text { OVERSEE QUALITY } \\
\text { ASSURRAMCE/OLALIIY } \\
\text { COHIROL ACTIVITIES }\end{array}$ & $\begin{array}{c}\text { OVERSEE LANO USE } \\
\text { ACIIVITIES } \\
\end{array}$ & $\begin{array}{c}\text { EVALUUIE } \\
\text { ALIERMAIIVES FOR } \\
\text { CLEAMUP } \\
\end{array}$ & $\begin{array}{l}\text { OVERSEE PROGRAN } \\
\text { EVALLATIOWS }\end{array}$ \\
\hline $\begin{array}{l}\text { Identifying relevant } \\
\text { imuterial/requirements }\end{array}$ & 1234 & 1234 & 1234 & 1234 & 1234 \\
\hline Delegating tasks & 1234 & 1234 & 1234 & 1234 & 1234 \\
\hline Defining applicable criteria & 1234 & 1234 & 1234 & 1234 & 1234 \\
\hline $\begin{array}{l}\text { Reading technical material for a } \\
\text { set purpose }\end{array}$ & 1234 & 1234 & 1234 & 1234 & 1234 \\
\hline Speed reading & 1234 & 1234 & 1236 & 1234 & 1234 \\
\hline Consol idating materials & 1234 & 1234 & 1234 & 1234 & 1234 \\
\hline $\begin{array}{l}\text { Examining trade-offs among } \\
\text { alternatives }\end{array}$ & 1234 & 1234 & 1234 & 1234 & 1234 \\
\hline $\begin{array}{l}\text { Geonapping, remote sensing, and } \\
\text { characterization techniques }\end{array}$ & 1234 & 1234 & 1234 & 1234 & 1234 \\
\hline $\begin{array}{l}\text { Proper engineering/technical } \\
\text { standards }\end{array}$ & 1234 & 1234 & 1234 & 1234 & 1234 \\
\hline Valid engineering solutions & 1234 & 1234 & 1234 & 1234 & 1234 \\
\hline Remediation techniques & 1234 & 1234 & $\begin{array}{llll}1 & 2 & 3 & 4 \\
\end{array}$ & 1234 & 1234 \\
\hline $\begin{array}{l}\text { Waste treatment/disposal } \\
\text { technologies }\end{array}$ & 1234 & $123 \quad 4 i$ & 1234 & 1234 & 1234 \\
\hline Hunan heal th impacts & 1234 & 1234 & 1234 & 1234 & 1234 \\
\hline Radiation effects & $123 \quad 3 \quad 4$ & 1234 & $123 \quad 4$ & 1234 & 1234 \\
\hline $\begin{array}{l}\text { Health and radiological safety } \\
\text { inpacts and benefits }\end{array}$ & 1234 & 1234 & 1234 & 1234 & 1234 \\
\hline Heal th physics & 1234 & 1234 & 1234 & 1234 & 1234 \\
\hline Sofety and health & 1234 & 1234 & 1234 & 1234 & 1234 \\
\hline Iransportation issues & 1234 & 1234 & 1234 & 1234 & 1234 \\
\hline Packaging issues & 1234 & 1234 & 1234 & 1234 & 1234 \\
\hline Biology, zoology, and ecology & 1234 & 1234 & 1234 & 1234 & 1234 \\
\hline Chemibtry & 1234 & 1234 & $123 \quad 4$ & 1234 & 1234 \\
\hline Huclear engineering & $\begin{array}{llll}1 & 2 & 3 & 4 \\
\end{array}$ & 1234 & 1234 & 1234 & 1234 \\
\hline
\end{tabular}


PART 2 - IDEAL SKILLS FOR EACH TASK

\begin{tabular}{|c|c|c|c|c|c|}
\hline IASKS $\cdots$ SKILLS/KHOMLEDCE & $\begin{array}{c}\text { ONERSEE } \\
\text { DECONTAHIMATION } \\
\text { DECOMISSIONIKG } \\
\end{array}$ & $\begin{array}{l}\text { OVERSEE DUALIIY } \\
\text { ASSURNCE/OUNLIIY } \\
\text { CONIROL ACTIVIYIIES } \\
\end{array}$ & $\begin{array}{l}\text { OVERSEE LAND USE } \\
\text { ACIIVITIES } \\
\end{array}$ & $\begin{array}{c}\text { EVALUAIE } \\
\text { M.TERMATIVES FOR } \\
\text { CLEAMUP } \\
\end{array}$ & $\begin{array}{c}\text { OVERSEE PROCRAM } \\
\text { EVALUATIOUS } \\
\end{array}$ \\
\hline Physical sciences & 1234 & 1234 & 1234 & 1234 & 1234 \\
\hline Geohydrology & 1234 & 1234 & 1234 & 1234 & 1234 \\
\hline Cartogruphy & 1234 & 1234 & 1234 & 1234 & 1234 \\
\hline Geophysics & 1234 & 1234 & 1234 & 1234 & 1234 \\
\hline D8D techniques & 1234 & 1234 & 1234 & 1234 & 1234 \\
\hline Cultural diversity issues & 1234 & 1234 & 1234 & 1234 & 1234 \\
\hline $\begin{array}{l}\text { Hazardous, chemical, radioactive } \\
\text { and mixed waste managenent }\end{array}$ & 1234 & 1234 & 1234 & 1234 & 1234 \\
\hline RCRA/CERCLA process & 1234 & 1234 & 1234 & 1234 & 1234 \\
\hline Hutural resources & 1234 & 1234 & 1234 & 1234 & 1234 \\
\hline Land use & 1234 & 1234 & 1234 & 1234 & 1234 \\
\hline Wildlife managenent & 1234 & 1234 & 1234 & 1234 & 1234 \\
\hline Soils, and minerals & 1234 & $123 \quad 34$ & 1234 & 1234 & 1234 \\
\hline Aquatic resources & $\begin{array}{llll}1 & 2 & 3 & 4 \\
\end{array}$ & 1234 & 1234 & 1234 & 1234 \\
\hline NEPA process & 1234 & 1234 & 1234 & 1234 & 1234 \\
\hline Enviromental sciences & 1234 & $1234^{1}$ & 1234 & 1234 & 1234 \\
\hline Quality assurance & 1234 & 1234 & 1234 & 1234 & 1234 \\
\hline Emergency response procedures & 1234 & 1234 & 1234 & 1234 & 1234 \\
\hline
\end{tabular}


PART 2 - IDEAL SKILLS FOR EACH TASK

\begin{tabular}{|c|c|c|c|c|c|}
\hline $\begin{array}{c}\text { TASKS } \cdots \text {-.. } \\
\text { SKILLS/KWOMEDEE }\end{array}$ & MAMAGE DONTRACTS & EVALUATE CONTRACTORS & $\begin{array}{l}\text { OVERSEE INDEPEMOEMT } \\
\text { VERIFICAYIOM } \\
\end{array}$ & $\begin{array}{l}\text { IDEMYIFY AND } \\
\text { ALLOCAIE RESOURCES } \\
\end{array}$ & $\begin{array}{l}\text { ProcuRE COCOS } 2 \\
\text { SERVICES }\end{array}$ \\
\hline Hational consensus standards & 1234 & 1234 & 1234 & 1234 & 1234 \\
\hline $\begin{array}{l}\text { Interugency agreements, } \\
\text { aduinistrution, and process }\end{array}$ & 1234 & 1234 & 1234 & 1234 & 1234 \\
\hline $\begin{array}{l}\text { Organizing information and } \\
\text { present cogent argunents }\end{array}$ & 1234 & 1234 & 1234 & 1234 & 1234 \\
\hline Writing succinctly and clearly & 1234 & 1234 & 1234 & 1234 & 1234 \\
\hline $\begin{array}{l}\text { Public speaking (e.g., public } \\
\text { presentation, employee/employer } \\
\text { interactions) }\end{array}$ & 1234 & $123 \quad 3 \quad 4$ & 1234 & 1234 & 1234 \\
\hline $\begin{array}{l}\text { Interpersonal facility (e.g., } \\
\text { public, medie, other employees } \\
\text { und special interest groups) }\end{array}$ & 1234 & 1234 & 1234 & 1234 & 1234 \\
\hline Hegotiating/conflict resolution & 1234 & 1234 & 1234 & 1234 & 1234 \\
\hline $\begin{array}{l}\text { Providing clear guidance and } \\
\text { direction to accomplish tasks }\end{array}$ & 1234 & 1234 & 1234 & 1234 & 1234 \\
\hline Meeting facilitation/leadership & 1234 & 1234 & 1234 & 1234 & 1234 \\
\hline OOE management strategies & 1234 & 1234 & 1234 & 1234 & 1234 \\
\hline $\begin{array}{l}\text { EH's mission, policies, } \\
\text { management plans, procedures, } \\
\text { priorities, goals ond objectives }\end{array}$ & 1234 & $\begin{array}{llll}1 & 2 & 3 & 4\end{array}$ & 1234 & 1234 & 1234 \\
\hline DOE departmental organization & 1234 & 1234 & 1234 & 1234 & 1234 \\
\hline $\begin{array}{l}\text { Contractor organization and } \\
\text { procedures }\end{array}$ & 1234 & 1234 & 1234 & 1234 & 1234 \\
\hline Decision analysis & 1234 & 1234 & 1234 & 1234 & 1234 \\
\hline Conducting library research & 1234 & 1234 & 1234 & 1234 & 1234 \\
\hline $\begin{array}{l}\text { Facility, site, operations and } \\
\text { processes }\end{array}$ & 1234 & 1234 & 1234 & 1234 & 1234 \\
\hline Defining boundaries of a problem & 1234 & 1234 & 1234 & 1234 & $123 \quad 4$ \\
\hline $\begin{array}{l}\text { Keeping abreast of current } \\
\text { developments }\end{array}$ & 1234 & 1234 & 1234 & 1234 & 1234 \\
\hline Organizing/categorizing & $\begin{array}{llll}1 & 2 & 3 & 4\end{array}$ & 1234 & 1234 & 1234 & 1234 \\
\hline
\end{tabular}


PART 2 - IDEAL SKILLS FOR EACH TASK

\begin{tabular}{|c|c|c|c|c|c|}
\hline rAsKS $\cdots$ r & & & & & \\
\hline SXILLS/KHOULEDCE & MUNAGE CONTRACTS & EVALUAYE COWIRACTOAS & $\begin{array}{l}\text { OVERSEE IYNEPEMDEMT } \\
\text { VERIFICAIION }\end{array}$ & $\begin{array}{l}\text { IDEMTIFY AND } \\
\text { ALLOCAIE RESOURCES }\end{array}$ & $\begin{array}{l}\text { PROCORE COOOS } 2 \\
\text { SERVICES }\end{array}$ \\
\hline $\begin{array}{l}\text { Operating equipnent (e.g., } \\
\text { testing equipnent, respirators) }\end{array}$ & 1234 & 1234 & 1234 & 1234 & 1234 \\
\hline Operating and using conputer & 1234 & 1234 & 1234 & 1234 & 1234 \\
\hline rouch typing & 1234 & 1234 & 1234 & 1234 & 1234 \\
\hline $\begin{array}{l}\text { Understanding principles of data } \\
\text { base requirements and basel ine } \\
\text { techniques }\end{array}$ & 1234 & 1234 & 1234 & 1234 & 1234 \\
\hline $\begin{array}{l}\text { Using sof tware and applying it } \\
\text { to tasks }\end{array}$ & 1234 & 1234 & 1234 & $\begin{array}{llll}1 & 2 & 3 & 4 \\
\end{array}$ & 1234 \\
\hline rime managentent & 1234 & 1234 & 1234 & 1234 & 1234 \\
\hline Heighing costs versus benefits & 1234 & 1234 & 1234 & 1234 & 1234 \\
\hline $\begin{array}{l}\text { Project or program management } \\
\text { techniques }\end{array}$ & 1234 & 1234 & 1234 & 1234 & 1234 \\
\hline $\begin{array}{l}\text { Principles of DOE budget } \\
\text { formulations, budget execution } \\
\text { and cost accounting, and the } \\
\text { procedures involved }\end{array}$ & 1234 & 1234 & 1234 & 1234 & 1234 \\
\hline Schedule constraints & 1234 & 1234 & 1234 & 1234 & 1234 \\
\hline $\begin{array}{l}\text { Planning, programing, funding } \\
\text { and acquisition management }\end{array}$ & 1234 & 1234 & 1234 & $123 \quad 3 \quad 4$ & 1234 \\
\hline $\begin{array}{l}\text { Conduct ing analyses involving } \\
\text { complex variables }\end{array}$ & 1234 & 1234 & 1234 & 1234 & $123 \quad 3 \quad 4$ \\
\hline Supervising staff/contractors & 1234 & 1234 & 1234 & 1234 & 1234 \\
\hline $\begin{array}{l}\text { Delegat ing authority and } \\
\text { responsibility }\end{array}$ & 1234 & 1234 & 1234 & 1234 & 1234 \\
\hline Equal Opportunity prograin plans & 1234 & 1234 & 1234 & 1234 & 1234 \\
\hline $\begin{array}{l}\text { Analyzing/synthesizing } \\
\text { information }\end{array}$ & 1234 & 1234 & 1234 & 1234 & 1234 \\
\hline $\begin{array}{l}\text { Assessing strengths and } \\
\text { Heaknesses of alternatives }\end{array}$ & 1234 & 1234 & 1234 & 1234 & $\begin{array}{llll}1 & 2 & 3 & 4 \\
\end{array}$ \\
\hline $\begin{array}{l}\text { Anticipating unintended } \\
\text { consequences }\end{array}$ & 1234 & 1234 & 1234 & 1234 & 1234 \\
\hline
\end{tabular}


PART 2 - IDEAL SKILLS FOR EACH TASK

\begin{tabular}{|c|c|c|c|c|c|}
\hline TASKS --.> & \multirow[b]{2}{*}{ MUACE CONTRACTS } & \multirow[b]{2}{*}{ EVALUATE CONTRACTORS } & \multirow[b]{2}{*}{$\begin{array}{l}\text { OVERSEE IMOEPENOENT } \\
\text { VERIFICATIOW }\end{array}$} & \multirow[b]{2}{*}{$\begin{array}{c}\text { IDEMIIFY AND } \\
\text { ALLOCATE RESOURCES } \\
\end{array}$} & \multirow[b]{2}{*}{$\begin{array}{l}\text { Procare COCOS } 2 \\
\text { SERVICES } \\
\end{array}$} \\
\hline SXILLS/KMOULEDE & & & & & \\
\hline $\begin{array}{l}\text { Lomparing existing conditions to } \\
\text { pre-set standards }\end{array}$ & 1234 & 1234 & 1234 & 1234 & 1234 \\
\hline Prioritizing based on criterio & 1234 & 1234 & 1234 & 1234 & 1234 \\
\hline $\begin{array}{l}\text { Identifying relevant } \\
\text { muterial/requlrements }\end{array}$ & 1234 & 1234 & 1234 & 1234 & 1234 \\
\hline Delegating tosks & 1234 & 1234 & 1234 & 1234 & 1234 \\
\hline Defining applicable criteria & 1234 & 1234 & $123 \quad 3 \quad 4$ & 1234 & 1234 \\
\hline $\begin{array}{l}\text { Reading technical material for a } \\
\text { set purpose }\end{array}$ & 1234 & 1234 & 1234 & 1234 & 1234 \\
\hline Speed reading & 1234 & 1234 & 1234 & 1234 & 1234 \\
\hline Consolidating materials & 1234 & 1234 & 1234 & 1234 & 1234 \\
\hline $\begin{array}{l}\text { Examining trade-offs among } \\
\text { olternatives }\end{array}$ & 1234 & 1234 & 1234 & 1234 & 1234 \\
\hline $\begin{array}{l}\text { Geomapping, remote sensing, and } \\
\text { characterization techniques }\end{array}$ & 1234 & 1234 & 1234 & $123 \quad 34$ & 1234 \\
\hline $\begin{array}{l}\text { Proper engineering/technical } \\
\text { standards }\end{array}$ & 1234 & 1234 & 1234 & 1234 & 1234 \\
\hline Valid engineering solutions & 1234 & 1234 & 1234 & 1234 & 1234 \\
\hline Remediation techniques & 1234 & 1234 & 1234 & 1234 & 1234 \\
\hline $\begin{array}{l}\text { Waste treatment/disposal } \\
\text { technologies }\end{array}$ & 1234 & 1234 & 1234 & 1234 & 1234 \\
\hline Human health impacts & 1234 & 1234 & 1234 & 1234 & 1234 \\
\hline Radiation effects & 1234 & 1234 & 1234 & 1234 & 1234 \\
\hline $\begin{array}{l}\text { Heal th and radiological safety } \\
\text { impacts and benefits }\end{array}$ & 1234 & 1234 & 1234 & 1234 & 1234 \\
\hline Heal th physics & 1234 & 1234 & 1234 & 1234 & 1234 \\
\hline Sofety and heolth & 1234 & 1234 & 1234 & 1234 & 1234 \\
\hline Iransportation issues & 1234 & 1234 & 1234 & 1234 & 1234 \\
\hline Packoging issues & 1234 & 1234 & 1234 & 1234 & 1234 \\
\hline
\end{tabular}


PART 2 - IDEAL SKILLS FOR EACH TASK

\begin{tabular}{|c|c|c|c|c|c|}
\hline IASKS $\quad \cdots$. & & & & & \\
\hline SKILLS/KMONLECE & MAMACE COWTRACTS & EVALUATE DOHTRACTORS & $\begin{array}{c}\text { OVERSEE INOEPEWOEUT } \\
\text { VERIFICAIIOH }\end{array}$ & $\begin{array}{c}\text { DDEKTIFY NOD } \\
\text { MLLOCAIE RESOURCES } \\
\end{array}$ & $\begin{array}{c}\text { PROCORE COOOS \& } \\
\text { SERVICES } \\
\end{array}$ \\
\hline Biology, zoology, and ecology & 1234 & 1234 & 1234 & 1234 & 1234 \\
\hline Chemistry & 1234 & 1234 & 1234 & 1234 & 1234 \\
\hline Nucl ear engineering & 1234 & 1234 & 1234 & 1234 & 1234 \\
\hline Physical sciences & 1234 & 1234 & 1234 & 1234 & 1234 \\
\hline Geohydrology & 1234 & 1234 & 1234 & 1234 & 1234 \\
\hline Cartography & 1234 & 1234 & 1234 & 1234 & 1234 \\
\hline Geophysics & 1234 & 1234 & 1234 & 1234 & 1234 \\
\hline D8D techniques & 1234 & 1234 & 1234 & 1234 & 1234 \\
\hline Cultural diversity issues & 1234 & 1234 & 1234 & 1234 & 1234 \\
\hline $\begin{array}{l}\text { Hazardous, chemical, radioactive } \\
\text { and mixed waste management }\end{array}$ & 1234 & 1234 & 1234 & 1234 & 1234 \\
\hline RCRA/CERCLA process & 1234 & 1234 & 1234 & 1234 & 1234 \\
\hline Natural resources & 1234 & 1234 & 1234 & 1234 & 1234 \\
\hline Land use & 1234 & 1234 & 1234 & 1234 & 1234 \\
\hline Hildlife management & 1234 & 1234 & 1234 & 1234 & 1234 \\
\hline Soils, and minerals & 1234 & 1234 & 1234 & 1234 & 1234 \\
\hline Aquatic resources & 1234 & 1234 & 1234 & 1234 & 1234 \\
\hline HEPA process & 1234 & 1234 & 1234 & 1234 & 1234 \\
\hline Enviromental sciences & 1234 & 1234 & 1234 & 1234 & 1234 \\
\hline Quality assurance & 1234 & 1234 & 1234 & 1234 & 1234 \\
\hline Emergency response procedures & 1234 & 1234 & 1234 & 1234 & 1234 \\
\hline
\end{tabular}


PART 2 - IDEAL SKILLS FOR EACH TASK

\begin{tabular}{|c|c|c|c|c|c|c|c|}
\hline$\frac{\text { TASKS } \cdots}{\text { SKILLS/KHOUREDCE }}$ & $\begin{array}{c}\text { OVERSEE } \\
\text { COST } \\
\text { REVIEUS } \\
\end{array}$ & $\begin{array}{r}\text { FORHULATE, } \\
\text { DEFENG, } \\
\text { MOIFY HLOCET } \\
\end{array}$ & $\begin{array}{l}\text { DEVELOP, } \\
\text { COORDIIRIE, } \\
\text { DISSERIMAIE } \\
\text { PROGRAM } \\
\text { MURAGEHENT } \\
\text { POLICIES L } \\
\text { PROCEOURES } \\
\end{array}$ & $\begin{array}{c}\text { ESTABLISH } \\
\text { OOST SCHEOULE } \\
\text { ANO IECHMICAL } \\
\text { BASELINES } \\
\end{array}$ & $\begin{array}{c}\text { DEVELOP } \\
\text { RESOURCE } \\
\text { RECUIRENENIS } \\
\text { (BOOCET/FTES) } \\
\end{array}$ & $\begin{array}{l}\text { DEVELOP } \\
\text { MEU/IIPROUE } \\
\text { IECHWOLOGIES } \\
\end{array}$ & $\begin{array}{c}\text { OVERSEE } \\
\text { ROOILLE } \\
\text { ACIIVITIES } \\
\end{array}$ \\
\hline National consensus standards & 1234 & $123 \quad 3 \quad 4$ & 1234 & 1234 & 1234 & 1234 & 1234 \\
\hline $\begin{array}{l}\text { Interogency agreements, } \\
\text { adninistration, and process }\end{array}$ & 1234 & 1234 & 1234 & 1234 & 1234 & 1234 & 1234 \\
\hline $\begin{array}{l}\text { Organizing information and } \\
\text { present cogent arguments }\end{array}$ & 1234 & 1234 & 1234 & 1234 & 1234 & 1234 & 1234 \\
\hline $\begin{array}{l}\text { Writing succinctly and } \\
\text { clearly }\end{array}$ & 1234 & 1234 & 1234 & 1234 & 1234 & 1234 & 1234 \\
\hline $\begin{array}{l}\text { Public speaking (e.g., } \\
\text { public presentations, } \\
\text { employee/employer } \\
\text { interactions) }\end{array}$ & 1234 & 1234 & 1234 & 1234 & 1234 & 1234 & $\begin{array}{llll}1 & 2 & 3 & 4\end{array}$ \\
\hline $\begin{array}{l}\text { Interpersonal facility } \\
\text { (e.g., public, media, other } \\
\text { employees and special } \\
\text { interest groups) }\end{array}$ & 1234 & 1234 & 1234 & 1234 & 1234 & 1234 & 1234 \\
\hline $\begin{array}{l}\text { Hegotiating/conflict } \\
\text { resolution }\end{array}$ & 1234 & 1234 & 1234 & 1234 & 1234 & 1234 & 1234 \\
\hline $\begin{array}{l}\text { Providing clear guidance and } \\
\text { direction to accomplish } \\
\text { tasks }\end{array}$ & 1234 & 1234 & $\begin{array}{rrr}1 & \\
123 \quad 4 \\
\end{array}$ & 1234 & 1234 & 1234 & $\begin{array}{llll}1 & 2 & 3 & 4 \\
\end{array}$ \\
\hline $\begin{array}{l}\text { Meeting } \\
\text { facilitation/leadership }\end{array}$ & 1234 & 1234 & 1234 & 1234 & 1234 & 1234 & $123 \quad 4$ \\
\hline DOE management strategies & 1234 & 1234 & 1234 & 1234 & $123 \quad 4$ & 1234 & 1234 \\
\hline $\begin{array}{l}\text { EH's mission, policies, } \\
\text { management plans, } \\
\text { procedures, priorities, } \\
\text { goals and objectives }\end{array}$ & 1234 & 1234 & 1234 & 1234 & 1234 & 1234 & $\begin{array}{llll}1 & 2 & 3 & 4 \\
\end{array}$ \\
\hline $\begin{array}{l}\text { DOE departmental } \\
\text { organization }\end{array}$ & $\begin{array}{llll}1 & 2 & 3 & 4\end{array}$ & $123 \quad 4$ & 1234 & 1234 & 1234 & 1234 & 1234 \\
\hline $\begin{array}{l}\text { Contractor organization and } \\
\text { procedures }\end{array}$ & 1234 & 1234 & 1234 & 1234 & 1234 & 1234 & 1234 \\
\hline Decision onalysis & 1234 & 1234 & 1234 & $\begin{array}{llll}1 & 2 & 3 & 4\end{array}$ & 1234 & 1234 & 1234 \\
\hline
\end{tabular}


PART 2 - IDEAL SKILLS FOR EACH TASK

\begin{tabular}{|c|c|c|c|c|c|c|c|}
\hline$\frac{\text { IASKS } \cdots}{\text { SKILLS/KHOALEDEE }}$ & $\begin{array}{c}\text { OVERSEE } \\
\text { COST } \\
\text { REVIEUS } \\
\end{array}$ & $\begin{array}{r}\text { FORWULATE, } \\
\text { DEFEHD, i } \\
\text { MOOIFY HUDCEI } \\
\end{array}$ & $\begin{array}{l}\text { DEVELOP, } \\
\text { COORDIMATE, } \\
\text { DISSEMIKAIE } \\
\text { PROCRUM } \\
\text { MUMACEKENT } \\
\text { POLICIES \& } \\
\text { PROCEDULES } \\
\end{array}$ & $\begin{array}{c}\text { ESTABLISH } \\
\text { COST SCHEOULE } \\
\text { AND TECHHICAL } \\
\text { UASELIHES } \\
\end{array}$ & $\begin{array}{c}\text { DEVELOP } \\
\text { RESOURCE } \\
\text { RECUIRENENTS } \\
\text { (EUDCEY/FTEY) } \\
\end{array}$ & $\begin{array}{c}\text { DEVELOP } \\
\text { MEU/IWWROVE } \\
\text { TECHMOLOGIES } \\
\end{array}$ & $\begin{array}{r}\text { OVERSEE } \\
\text { RODILE } \\
\text { ACIIVIIIES } \\
\end{array}$ \\
\hline Conducting library research & 1234 & 1234 & 1234 & 1234 & 1234 & 1234 & 1234 \\
\hline $\begin{array}{l}\text { Facllity, site, operations } \\
\text { and processes }\end{array}$ & 1234 & 1234 & 1234 & 1234 & 1234 & 1234 & 1234 \\
\hline $\begin{array}{l}\text { Defining boundaries of a } \\
\text { problem }\end{array}$ & 1234 & 1234 & 1234 & 1234 & 1234 & 1234 & 1234 \\
\hline $\begin{array}{l}\text { Keeping abreast of current } \\
\text { developments }\end{array}$ & 1234 & 1234 & $123 \quad 3 \quad 4$ & 1234 & 1234 & 1234 & 1234 \\
\hline orgenizing/categorizing & 1234 & 1234 & 1234 & 1234 & 1234 & 1234 & 1234 \\
\hline $\begin{array}{l}\text { Operating equipment (e.g., } \\
\text { testing, equipment, } \\
\text { respirators) }\end{array}$ & 1234 & 1234 & 1234 & 1234 & 1234 & 1234 & 1234 \\
\hline Operating and using computer & 1234 & 1234 & 1234 & 1234 & 1234 & 1234 & 1234 \\
\hline Touch typing & 1234 & 1234 & 1234 & 1234 & 1234 & 1234 & 1234 \\
\hline $\begin{array}{l}\text { Understanding principles of } \\
\text { data base requirements and } \\
\text { basel ine techniques }\end{array}$ & 1234 & 1234 & 1234 & 1234 & 1234 & 1234 & 1234 \\
\hline $\begin{array}{l}\text { Using software and applying } \\
\text { it to tasks }\end{array}$ & 1234 & 1234 & 1234 & 1234 & 1234 & 1234 & 1234 \\
\hline I ime management & 1234 & 1234 & 1234 & 1234 & 1234 & 1234 & 1234 \\
\hline $\begin{array}{l}\text { Weighing costs versus } \\
\text { benefits }\end{array}$ & 1234 & 1234 & 1234 & 1234 & 1234 & 1234 & 1234 \\
\hline $\begin{array}{l}\text { Project or program } \\
\text { management techniques }\end{array}$ & 1234 & 1234 & 1234 & 1234 & 1234 & 1234 & 1234 \\
\hline $\begin{array}{l}\text { Principles of DoE budget } \\
\text { formulations, budget } \\
\text { execution and cost } \\
\text { account ing, and the } \\
\text { procedures involved }\end{array}$ & 1234 & $\begin{array}{llll}1 & 2 & 3 & 4 \\
\end{array}$ & 1234 & 1234 & 1234 & 1234 & 1234 \\
\hline Schedule constraints & 1234 & 1234 & 1234 & $\begin{array}{llll}12 & 3 & 4\end{array}$ & 1234 & 1234 & 1234 \\
\hline
\end{tabular}


PART 2 - IDEAL SKILLS FOR EACH TASK

\begin{tabular}{|c|c|c|c|c|c|c|c|}
\hline $\begin{array}{c}\text { TASKS } \cdots \text {-..> } \\
\text { SKILLS/KwanLEDCE }\end{array}$ & $\begin{array}{c}\text { OVERSEE } \\
\text { COST } \\
\text { REVIEUS } \\
\end{array}$ & $\begin{array}{c}\text { FORNULATE, } \\
\text { DEFENO, } \\
\text { MOOIFY BLOCET } \\
\end{array}$ & $\begin{array}{l}\text { DEVELCP, } \\
\text { DOOROIIAIE, } \\
\text { DISSEMIMATE } \\
\text { PROGRAM } \\
\text { MAXACENENT } \\
\text { POLICIES 2 } \\
\text { PROCEROURES } \\
\end{array}$ & $\begin{array}{l}\text { ESTABLISH } \\
\text { COST SCHEDULE } \\
\text { AHD TECHWICAL } \\
\text { BASELIKES } \\
\end{array}$ & $\begin{array}{c}\text { OEVELOP } \\
\text { RESOURCE } \\
\text { RECUIREWENTS } \\
\text { (BUDGET/FIES) } \\
\end{array}$ & $\begin{array}{c}\text { DEVELLP } \\
\text { MEW/IMPRONE } \\
\text { IECHWOLOGIES } \\
\end{array}$ & $\begin{array}{c}\text { OVERSEE } \\
\text { PODILE } \\
\text { ACTIVIIIIES } \\
\end{array}$ \\
\hline $\begin{array}{l}\text { funding and ocquisition } \\
\text { management }\end{array}$ & 1234 & 1234 & 1234 & 1234 & 1234 & 1234 & 1234 \\
\hline $\begin{array}{l}\text { Conducting analyses } \\
\text { involving complex variables }\end{array}$ & 1234 & 1234 & 1234 & 1234 & 1234 & 1234 & 1234 \\
\hline $\begin{array}{l}\text { Supervising } \\
\text { staff/contractors }\end{array}$ & 1234 & 1234 & 1234 & 1234 & 1234 & 1234 & 1234 \\
\hline $\begin{array}{l}\text { Delegating authority and } \\
\text { responsibility }\end{array}$ & 1234 & 1234 & 1234 & 1234 & 1234 & 1234 & $\begin{array}{llll}123 & 3 \\
\end{array}$ \\
\hline $\begin{array}{l}\text { Equal Opportunity program } \\
\text { plans }\end{array}$ & 1234 & 1234 & 1234 & 1234 & 1234 & 1234 & 1234 \\
\hline $\begin{array}{l}\text { Analyzing/synthesizing } \\
\text { information }\end{array}$ & 1234 & 1234 & 1234 & 1234 & 1234 & 1234 & $\begin{array}{llll}1 & 2 & 3 & 4\end{array}$ \\
\hline $\begin{array}{l}\text { Assessing strengths and } \\
\text { Heaknesses of al ternatives }\end{array}$ & 1234 & 1234 & 1234 & 1234 & 1234 & 1234 & 1234 \\
\hline $\begin{array}{l}\text { Anticipating unintended } \\
\text { consequences }\end{array}$ & 1234 & 1234 & 12,34 & 1234 & 1234 & 1234 & 1234 \\
\hline $\begin{array}{l}\text { Comparing existing } \\
\text { conditions to pre-set } \\
\text { standards }\end{array}$ & 1234 & 1234 & 1234 & 1234 & 1234 & 1234 & $\begin{array}{llll}1 & 2 & 3 & 4 \\
\end{array}$ \\
\hline $\begin{array}{l}\text { Prioritizing based on } \\
\text { criterio }\end{array}$ & 1234 & 1234 & 1234 & 1234 & 1234 & 1234 & 1234 \\
\hline $\begin{array}{l}\text { Identifying relevant } \\
\text { material/requirements }\end{array}$ & 1234 & 1234 & 1234 & 1234 & 1234 & 1234 & 1234 \\
\hline Delegating tasks & 1234 & 1234 & 1234 & 1234 & 1234 & 1234 & 1234 \\
\hline Defining applicable criteria & 1234 & 1234 & 1234 & 1234 & 1234 & 1234 & 1234 \\
\hline $\begin{array}{l}\text { Reading technical material } \\
\text { for a set purpose }\end{array}$ & 1234 & 1234 & 1234 & 1234 & 1234 & 1234 & 1234 \\
\hline Speed reading & 1234 & 1234 & 1234 & 1234 & 1234 & 1234 & 1234 \\
\hline Consol idating materials & 1234 & 1234 & 1234 & 1234 & 1234 & 1234 & 1234 \\
\hline
\end{tabular}


PART 2 - IDEAL SKILLS FOR EACH TASK

\begin{tabular}{|c|c|c|c|c|c|c|c|}
\hline$\frac{\text { TASKS } \cdots>}{\text { SKILLS/KWOALEDCE }}$ & $\begin{array}{l}\text { OVERSEE } \\
\text { COST } \\
\text { REVIEUS }\end{array}$ & $\begin{array}{l}\text { FORMULATE, } \\
\text { DEFEWO, } \\
\text { MOOIFY UIOGEY } \\
\end{array}$ & $\begin{array}{l}\text { DEVELLP, } \\
\text { COORDIIAIE, } \\
\text { DISSEMIMAIE } \\
\text { PROCRAH } \\
\text { MURACENENT } \\
\text { POLICIES \& } \\
\text { PROCEDUURES } \\
\end{array}$ & $\begin{array}{c}\text { ESTABLISH } \\
\text { COST SCHEDULE } \\
\text { AND IECHWICAL. } \\
\text { UASELIKES } \\
\end{array}$ & $\begin{array}{c}\text { DEVELOP } \\
\text { RESOURCE } \\
\text { REQUIREMENTS } \\
\text { (BLOCEY/FYES) } \\
\end{array}$ & $\begin{array}{c}\text { DEVELLP } \\
\text { MEWIIMPROVE } \\
\text { IECHMOLOGIES } \\
\end{array}$ & $\begin{array}{r}\text { OVERSEE } \\
\text { RDOILEE } \\
\text { ACIIVIIIES } \\
\end{array}$ \\
\hline $\begin{array}{l}\text { Examining trade-offs among } \\
\text { ol ternatives }\end{array}$ & 1234 & 1234 & 1234 & 1234 & 1234 & 1234 & 1234 \\
\hline $\begin{array}{l}\text { Geomapping, remote sensing, } \\
\text { and characterization } \\
\text { techniques }\end{array}$ & 1234 & 1234 & 1234 & 1234 & 1234 & 1234 & 1234 \\
\hline $\begin{array}{l}\text { Proper engineering/technical } \\
\text { standerds }\end{array}$ & 1234 & 1234 & 1234 & 1234 & 1234 & 1234 & 1234 \\
\hline Valid engineering solutions & 1234 & 1234 & 1234 & 1234 & 1234 & 1234 & 1234 \\
\hline Remediation techniques & 1234 & 1234 & 1234 & 1234 & 1234 & 1234 & 1234 \\
\hline $\begin{array}{l}\text { Waste treatment/disposal } \\
\text { technologies }\end{array}$ & 1234 & 1234 & 1234 & 1234 & 1234 & $123 \quad 3 \quad 4$ & 1234 \\
\hline Human heal th impacts & 1234 & 1234 & 1234 & 1234 & 1234 & 1234 & 1234 \\
\hline Radiation effects & 1234 & 1234 & 1234 & 1234 & 1234 & 1234 & 1234 \\
\hline $\begin{array}{l}\text { Heal th and radiological } \\
\text { safety impacts and benefits }\end{array}$ & 1234 & 1234 & 1234 & 1234 & 1234 & 1234 & $123 \quad 4$ \\
\hline Heal th physics & 1234 & 1234 & 12,34 & 1234 & 1234 & 1234 & 1234 \\
\hline Safety and heal th & 1234 & 1234 & 1234 & 1234 & 1234 & 1234 & 1234 \\
\hline Iransportation issues & 1234 & 1234 & 1234 & 1234 & 1234 & 1234 & 1234 \\
\hline Packaging issues & 1234 & 1234 & 1234 & 1234 & 1234 & 1234 & 1234 \\
\hline $\begin{array}{l}\text { Biology, } 200 \operatorname{logy} \text {, and } \\
\text { ecology }\end{array}$ & $123 \quad 34$ & 1234 & 1234 & 1234 & 1234 & 1234 & 1234 \\
\hline Chemistry & 1234 & 1234 & 1234 & 1234 & 1234 & 1234 & 1234 \\
\hline Nuclear engineering & 1234 & 1234 & 1234 & 1234 & 1234 & 1234 & 1234 \\
\hline Physical sciences & 1234 & 1234 & 1234 & 1234 & 1234 & 1234 & 1234 \\
\hline Geohydrology & $123 \quad 34$ & 1234 & 1234 & 1234 & 1234 & 1234 & 1234 \\
\hline Cartography & 1234 & 1234 & 1234 & 1234 & 1234 & 1234 & 1234 \\
\hline Geophysics & 1234 & 1234 & 1234 & 1234 & 1234 & 1234 & 1234 \\
\hline
\end{tabular}


PART 2 - IDEAL SKILLS FOR EACH TASK

\begin{tabular}{|c|c|c|c|c|c|c|c|}
\hline $\begin{array}{c}\text { TASKS } \quad-->> \\
\text { SKILLS/KWOULEDGE }\end{array}$ & $\begin{array}{c}\text { OVERSEE } \\
\text { COSI } \\
\text { REVIEUS } \\
\end{array}$ & $\begin{array}{l}\text { FORMULATE, } \\
\text { DEFEND, \& } \\
\text { MOOIFY BUOCET }\end{array}$ & 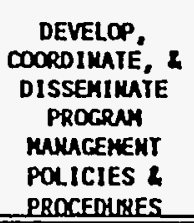 & $\begin{array}{l}\text { ESTABLISH } \\
\text { COST SCHEDULE } \\
\text { NO IECHNICAL } \\
\text { BASELINES } \\
\end{array}$ & $\begin{array}{l}\text { DEVELOP } \\
\text { RESOURCE } \\
\text { RECUIREYEMTS } \\
\text { (BUDCET/FIEM) } \\
\end{array}$ & $\begin{array}{l}\text { DEVELLP } \\
\text { MEU/IMPROVE } \\
\text { IECHMOLOGIES }\end{array}$ & $\begin{array}{c}\text { OVERSEE } \\
\text { RODILE } \\
\text { ACIIVITIES }\end{array}$ \\
\hline D8D techniques & 1234 & 1234 & 1234 & 1234 & 1234 & 1234 & 1234 \\
\hline Cul tural diverslty issues & 1234 & 1234 & 1234 & 1234 & 1234 & 1234 & 1234 \\
\hline $\begin{array}{l}\text { Hazardous, chemical, } \\
\text { radioactive and mixed waste } \\
\text { management }\end{array}$ & 1234 & $123 \quad 3 \quad 4$ & 1234 & 1234 & 1234 & 1234 & 1234 \\
\hline RCRA/CERCLA process & 1234 & 1234 & 1234 & 1234 & 1234 & 1234 & 1234 \\
\hline Hatural resources & 1234 & 1234 & 1234 & 1234 & 1234 & 1234 & 1234 \\
\hline Land use & 1234 & 1234 & 1234 & 1234 & 1234 & 1234 & 1234 \\
\hline Wildl ife management & 1234 & 1234 & 1234 & 1234 & $\begin{array}{llll}1 & 2 & 3 & 4\end{array}$ & 1234 & 1234 \\
\hline Soils, and minerals & 1234 & 1234 & 1234 & 1234 & 1234 & 1234 & 1234 \\
\hline Aquat ic resources & 1234 & 1234 & 1234 & 1234 & 1234 & 1234 & 1234 \\
\hline MEPA process & 1234 & 1234 & 1234 & 1234 & 1234 & 1234 & 1234 \\
\hline Enviromental sciences & 1234 & 1234 & 1234 & $\begin{array}{llll}1 & 2 & 3 & 4\end{array}$ & 1234 & 1234 & 1234 \\
\hline Quality assurance & 1234 & 1234 & 1234 & 1234 & 1234 & 1234 & 1234 \\
\hline $\begin{array}{l}\text { Emergency response } \\
\text { procedures }\end{array}$ & 1234 & 1234 & 1234 & $\begin{array}{llll}1 & 2 & 3 & 4\end{array}$ & 1234 & 1234 & 1234 \\
\hline
\end{tabular}


A-63

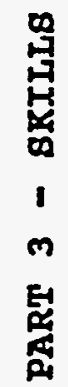

a

究夜 


\section{GENERAL KNOWLEDGE AND SKILLS}

National consensus standards

$\begin{array}{llll}1 & 2 & 3 & 4\end{array}$

Interagency agreements, administration, and process

12534

Organizing information and present cogent arguments

$\begin{array}{llll}1 & 2 & 3 & 4\end{array}$

Hriting succinctly and clearly

$\begin{array}{llll}1 & 2 & 3 & 4\end{array}$

Public speaking (e.g., public presentations,

$\begin{array}{llll}1 & 2 & 3 & 4\end{array}$ employee/employer interactions)

$\begin{array}{llll}1 & 2 & 3 & 4\end{array}$

Interpersonal facility (e.g., public, media, other employees and special interest groups)

Negotiating/conflict resolution

$\begin{array}{llll}1 & 2 & 3 & 4\end{array}$

Providing clear guidance and direction to accomplish tasks

1234

Meeting facilitation/leadership

DOE management strategies

EM's mission, policies, management plans, procedures, priorities, goals and objectives

$\begin{array}{llll}1 & 2 & 3 & 4\end{array}$

$\begin{array}{llll}1 & 2 & 3 & 4\end{array}$

$\begin{array}{llll}1 & 2 & 3 & 4\end{array}$

DOE departmental organization

$\begin{array}{llll}1 & 2 & 3 & 4\end{array}$

Contractor organization and procedures

$\begin{array}{llll}1 & 2 & 3 & 4\end{array}$

Decision analysis

$\begin{array}{llll}1 & 2 & 3 & 4\end{array}$ 
Conducting library research

Facility, site, operations and processes

Defining boundaries of a problem

Keeping abreast of current developments

Organizing/categorizing

Operating equipment (e.g., testing equipment, respirators)

Operating and using computer

Touch typing

Understanding principles of data base requirements and baseline techniques

Using software and applying it to tasks

Time management

Weighing costs versus benefits

Project or program management techniques

Principles of DOE budget formulations, budget

execution and cost accounting, and the procedures involved

Schedule constraints

Planning, programming, funding and acquisition management $\begin{array}{llll}1 & 2 & 3 & 4\end{array}$

$\begin{array}{llll}1 & 2 & 3 & 4\end{array}$

$\begin{array}{llll}1 & 2 & 3 & 4\end{array}$

$\begin{array}{llll}1 & 2 & 3 & 4\end{array}$

$\begin{array}{llll}1 & 2 & 3 & 4\end{array}$

$\begin{array}{llll}1 & 2 & 3 & 4\end{array}$

$\begin{array}{llll}1 & 2 & 3 & 4\end{array}$

$\begin{array}{llll}1 & 2 & 3 & 4\end{array}$

1234

1234

1234

1234

1234

1234

1234

1234 
Conducting analyses involving complex variables

Supervising staff

Delegating authority and responsibility

Equal Opportunity program plans

Analyzing information

Assessing strengths and weaknesses of alternatives

Anticipating unintended consequences

Comparing existing conditions to pre-set standards

Prioritizing based on criteria

Identifying relevant material/requirements

Delegating tasks

Defining applicable criteria

Reading technical material for a set purpose

Speed reading

Consolidating materials

Examining trade-offs among alternatives $\begin{array}{llll}1 & 2 & 3 & 4\end{array}$

$\begin{array}{llll}1 & 2 & 3 & 4\end{array}$

$\begin{array}{llll}1 & 2 & 3 & 4\end{array}$

$\begin{array}{llll}1 & 2 & 3 & 4\end{array}$

$\begin{array}{llll}1 & 2 & 3 & 4\end{array}$

$\begin{array}{llll}1 & 2 & 3 & 4\end{array}$

$\begin{array}{llll}1 & 2 & 3 & 4\end{array}$

$\begin{array}{llll}1 & 2 & 3 & 4\end{array}$

$\begin{array}{llll}1 & 2 & 3 & 4\end{array}$

$\begin{array}{llll}1 & 2 & 3 & 4\end{array}$

$\begin{array}{llll}1 & 2 & 3 & 4\end{array}$

1234

$\begin{array}{llll}1 & 2 & 3 & 4\end{array}$

1234

1234

1234

6/29/93

M6319 
TECHNICAL KNOWLEDGE

Geomapping, remote sensing techniques

$\begin{array}{llll}1 & 2 & 3 & 4\end{array}$

Proper engineering/technical standards

1234

Valid engineering solutions

1234

Remediation techniques

$\begin{array}{llll}1 & 2 & 3 & 4\end{array}$

Waste treatment/disposal technologies

1234

Human health impacts

$\begin{array}{llll}1 & 2 & 3 & 4\end{array}$

Radiation effects

$\begin{array}{llll}1 & 2 & 3 & 4\end{array}$

Health and radiological safety impacts and benefits

$123 \quad 4$

Health physics

$123 \quad 4$

Safety and Health

$123 \quad 4$

Transportation issues

$\begin{array}{llll}1 & 2 & 3 & 4\end{array}$

Packaging issues

$\begin{array}{llll}1 & 2 & 3 & 4\end{array}$

Biology, zoology, and ecology

1234

Chemistry

$\begin{array}{llll}1 & 2 & 3 & 4\end{array}$

Nuclear engineering

1234

- Physical sciences

1234

$6 / 29 / 93$

$M 6319$ 
PART 3 - BRIILS

\begin{tabular}{|c|c|c|c|}
\hline Geohydrology & 1 & 2 & 3 \\
\hline Cartography & 1 & 2 & 3 \\
\hline Geophysics & 1 & 2 & 3 \\
\hline D\&D techniques & 1 & 2 & 3 \\
\hline Cultural diversity issues & 1 & 2 & 3 \\
\hline Hazardous, chemical, radioactive and mixed waste management & 1 & 2 & 3 \\
\hline RCRA/CERCLA process & 1 & 2 & 3 \\
\hline Natural resources & 1 & 2 & 3 \\
\hline Land use & 1 & 2 & 3 \\
\hline Wildlife management & 1 & 2 & 3 \\
\hline Soils, and minerals & 1 & 2 & 3 \\
\hline Aquatic resources & 1 & 2 & 3 \\
\hline NEPA process & 1 & 2 & 3 \\
\hline Environmental sciences & 1 & 2 & 3 \\
\hline Emergency response procedures & 1 & 2 & 3 \\
\hline Quality assurance & 1 & 2 & 3 \\
\hline
\end{tabular}




\section{REGULATORY KNOWLEDGE}

Laws and/or Their Implementing Requlations

American Indian Religious Freedom Act

$123 \quad 3 \quad 4$

Archaeological and Historic Preservation Act

$\begin{array}{llll}1 & 2 & 3 & 4\end{array}$

Archaeological Resources Protection Act

$123 \quad 4$

Atomic Energy Act of 1954 as amended

Clean Air Act as Amended in 1990

Comprehensive Environmental Response, Compensation and Liability Act

$\begin{array}{llll}1 & 2 & 3 & 4\end{array}$

$\begin{array}{llll}1 & 2 & 3 & 4\end{array}$

$\begin{array}{llll}1 & 2 & 3 & 4\end{array}$

Clean Water Act as amended

$\begin{array}{llll}1 & 2 & 3 & 4\end{array}$

Coastal Zone Management Act

$\begin{array}{llll}1 & 2 & 3 & 4\end{array}$

Emergency Planning and Community Right-to-Know Act ,

$\begin{array}{llll}1 & 2 & 3 & 4\end{array}$

Endangered Species Act

1234

Federal Insecticide, Fungicide, and Rodenticide Act

12234

Fish and Wildlife Coordination Act

$123 \quad 4$

International Maritime Act

Marine Mammal Protection Act

$\begin{array}{llll}1 & 2 & 3 & 4\end{array}$

$\begin{array}{llll}1 & 2 & 3 & 4\end{array}$ 
PART 3 - BRILLB

Marine Protection, Research, and Sanctuaries Act

Migratory Bird Treaty Act

National Environmental Policy Act

National Historic Preservation Act

National Wildlife Refuge System Administration Act

Native American Graves Protection and Repatriation Act

Noise Control Act

Noxious Weed Act

Nuclear Waste Policy Act

Occupational Safety and Health Act

0il Pollution Act

Pollution Prevention Act

Prime Farml and Act

Resource Conservation and Recovery Act

Repatriation Act

Rivers and Harbors Act

Safe Drinking Water Act $\begin{array}{llll}1 & 2 & 3 & 4\end{array}$

$\begin{array}{llll}1 & 2 & 3 & 4\end{array}$

$\begin{array}{llll}1 & 2 & 3 & 4\end{array}$

$\begin{array}{llll}1 & 2 & 3 & 4\end{array}$

1234

$\begin{array}{llll}1 & 2 & 3 & 4\end{array}$

1234

$\begin{array}{llll}1 & 2 & 3 & 4\end{array}$

$\begin{array}{llll}1 & 2 & 3 & 4\end{array}$

$\begin{array}{llll}1 & 2 & 3 & 4\end{array}$

$\begin{array}{llll}1 & 2 & 3 & 4\end{array}$

$\begin{array}{llll}1 & 2 & 3 & 4\end{array}$

$\begin{array}{llll}1 & 2 & 3 & 4\end{array}$

1234

1234

1234

$\begin{array}{llll}1 & 2 & 3 & 4\end{array}$ 
Toxic Substances Control Act

Wild and Scenic Rivers Act

Wild Horse and Burros Act

DOE Orders

1324.3 Files Management

1324.5A Records Management Program

1500.2A Travel Policy and Procedures

1500.3 Foreign Travel Authorization

1600.3 Policy on Sexual Harassment

1700.1 Freedom of Information Program

2200.6A Financial Accounting

2200.7 Cost Accounting

2300.1B Audit Resolution and Follow-up

3410.1B Training

3430.3A Departmental Performance Appraisal System

3600.1B Time and Attendance Reporting

3630.1B Leave Administration
$\begin{array}{llll}1 & 2 & 3 & 4\end{array}$
$\begin{array}{llll}1 & 2 & 3 & 4\end{array}$
$\begin{array}{llll}1 & 2 & 3 & 4\end{array}$
$\begin{array}{llll}1 & 2 & 3 & 4\end{array}$
$\begin{array}{llll}1 & 2 & 3 & 4\end{array}$
$\begin{array}{llll}1 & 2 & 3 & 4\end{array}$
$\begin{array}{llll}1 & 2 & 3 & 4\end{array}$
$\begin{array}{llll}1 & 2 & 3 & 4\end{array}$
$\begin{array}{llll}1 & 2 & 3 & 4\end{array}$
$\begin{array}{llll}1 & 2 & 3 & 4\end{array}$
$\begin{array}{llll}1 & 2 & 3 & 4\end{array}$
$\begin{array}{llll}1 & 2 & 3 & 4\end{array}$
1234
1234
1234
$\begin{array}{llll}1 & 2 & 3 & 4\end{array}$ 
3790.2A Federal Employee Occupational Safety and

4300.1C Real Property Management

$\begin{array}{llll}1 & 2 & 3 & 4\end{array}$

4320.1B Site Development Planning

4700.1 Project Management System \& Associate Notices

5000.3B Occurrence Reporting and Processing of Operations Information

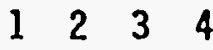

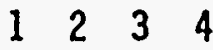

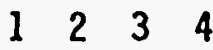

5400.1 General Environmental Protection Program

$\begin{array}{llll}1 & 2 & 3 & 4\end{array}$

5400.2A Environmental Compliance Issue Coordination

5400.3 Hazardous and Radioactive Mixed Waste Program

5400.4 Comprehensive Environmental Response, Compensation and Liability Act Requirements

5400.5 Radiation Protection of the Public and the Environment

$\begin{array}{llll}1 & 2 & 3 & 4\end{array}$

5440.1E National Environmental Policy Act Compliance Program

$\begin{array}{llll}1 & 2 & 3 & 4\end{array}$

5480.1B Environment, Safety and Health Program for

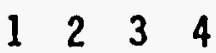
Department of Energy Operations

5480.3 Safety Requirements for the Packaging and Transportation of Hazardous Materials, Hazardous Substances, and Hazardous Wastes 
5480.4 Environmental Protection, Safety and Health

5480.5 Safety of Nuclear Facilities

$\begin{array}{llll}1 & 2 & 3 & 4\end{array}$

5480.6 Radiological Control and Radiological

$\begin{array}{llll}1 & 2 & 3 & 4\end{array}$

Control Manual

5480.11 Radiation Protection for Occupational Workers

5480.15 Department of Energy Laboratory

Accreditation Program for Personnel Dosimetry

$123 \quad 4$

$\begin{array}{llll}1 & 2 & 3 & 4\end{array}$

5480.19 Conduct of Operations Requirements for

$\begin{array}{llll}1 & 2 & 3 & 4\end{array}$

5480.22 Technical Safety Requirements

$\begin{array}{llll}1 & 2 & 3 & 4\end{array}$

5480.23 Nuclear Safety Analysis Reports

$\begin{array}{llll}1 & 2 & 3 & 4\end{array}$

5481.1B Safety Analysis and Review System

$\begin{array}{llll}1 & 2 & 3 & 4\end{array}$

5482.1B Environment, Safety, and Health Appraisal Program

$\begin{array}{llll}1 & 2 & 3 & 4\end{array}$

5482.1A Occupational Safety and Health Program

$123 \quad 4$

for DOE Contractor Employees at Government-Owned Contractor-Operated Facilities

5484.1 Environmental Protection, Safety, and Health Protection Information Reporting Requirements

5500.1B Emergency Management System 
PART 3 - 8KILLB

5500.2B Emergency Categories, Classes and

Notification and Reporting Requirements

12234

5820.2A Radioactive Waste Management

$\begin{array}{llll}1 & 2 & 3 & 4\end{array}$

6430.1A General Design Criteria

$\begin{array}{llll}1 & 2 & 3 & 4\end{array}$

Executive Orders

11514 Protection and Enhancement of Environmental Quality

$\begin{array}{llll}1 & 2 & 3 & 4\end{array}$

11593 Protection and Enhancement of the Cultural Environment

$\begin{array}{llll}1 & 2 & 3 & 4\end{array}$

11738 Providing for the Administration of the Clean Air Act and the Federal Water Pollution Control Act

$\begin{array}{llll}1 & 2 & 3 & 4\end{array}$ with Respect to Federal Contracts, Grants of Loans

11987 Exotic Organisms

$\begin{array}{llll}1 & 2 & 3 & 4\end{array}$

$11988 \quad$ Floodplain Management

$\begin{array}{llll}1 & 2 & 3 & 4\end{array}$

11989 Off-Road Vehicles on Public Lands

$\begin{array}{llll}1 & 2 & 3 & 4\end{array}$

11990 Protection of Wetlands

$\begin{array}{llll}1 & 2 & 3 & 4\end{array}$

12088 Federal Compliance with Pollution Control Standards

$\begin{array}{llll}1 & 2 & 3 & 4\end{array}$

12196 Federal Compliance with Occupational Safety

$1253 \quad 4$

12777. Implementation of Section 311 of the Federal

$\begin{array}{llll}1 & 2 & 3 & 4\end{array}$ 
PART 3 - BKILLB

Water Pollution Control Act of 1972, as amended, and the 0il Pollution Act of

12780 Federal Agency Recycling and the Council on Federal Recycling and Procurement Policy

Secretary of Energy Notices

$\begin{array}{llll}1 & 2 & 3 & 4\end{array}$ 

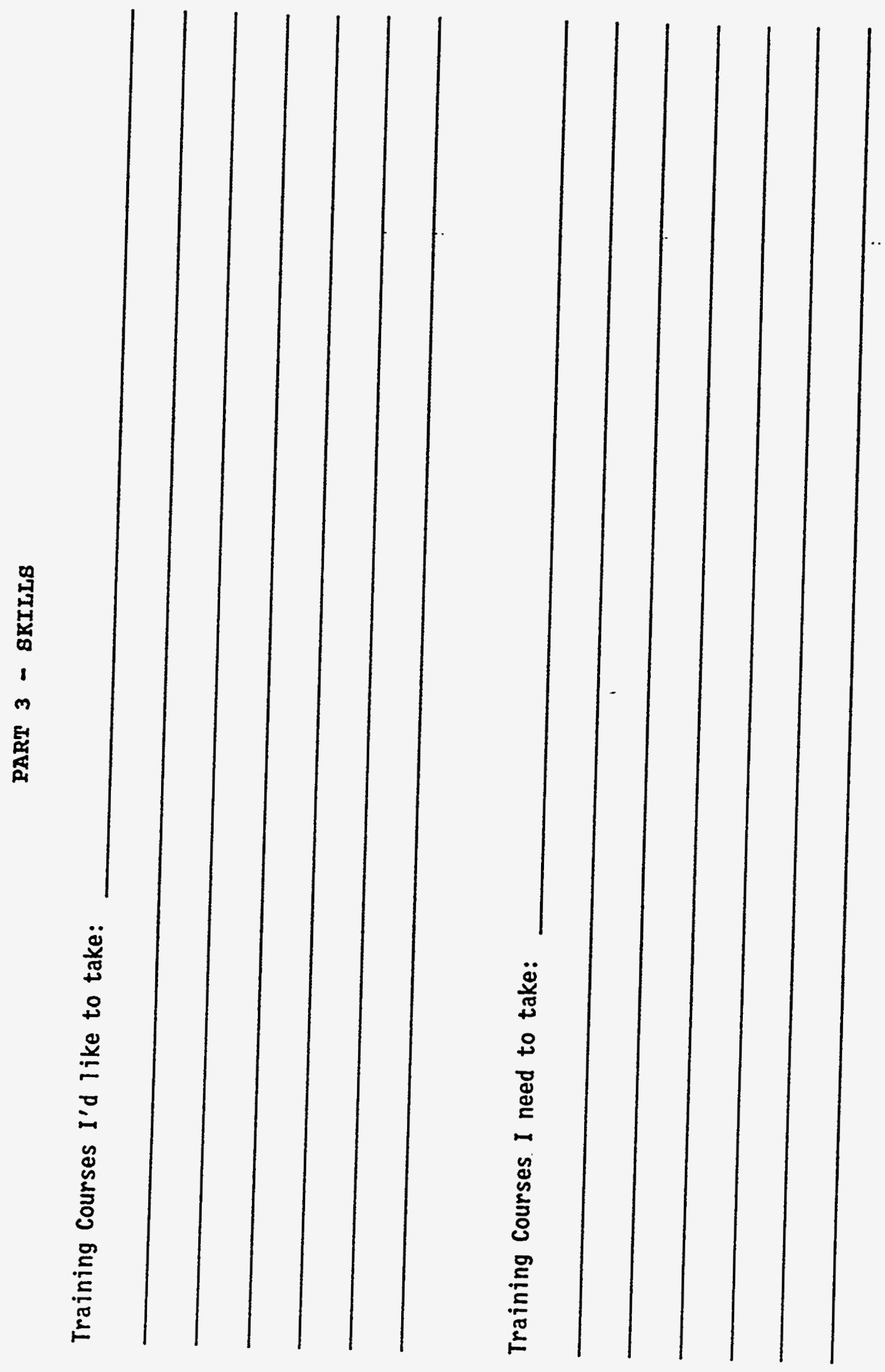

㠃高 
$B-1$

APPENDIX B:

\section{MOST CRITICAL SKILLS FOR EACH TASK}


B-2 
TABLE B.1 Most Critical Skills ${ }^{\text {a }}$ Needed to Prepare Documents

\begin{tabular}{lc}
\hline \multicolumn{1}{c}{ Skills } & Score ${ }^{\mathrm{b}}$ \\
\hline Writing succinctly and clearly & 3.79 \\
Keeping abreast of current developments & 3.58 \\
Organizing information and presenting cogent arguments & 3.53 \\
EM's mission, policies, management plans, procedures, priorities, goals, and objectives & 3.47 \\
Defining boundaries of a problem & 3.47 \\
Analyzing information & 3.44 \\
Assessing strengths and weaknesses of alternatives & 3.44 \\
RCRA/CERCLA process & 3.41 \\
Organizing/categorizing & 3.39 \\
Time management & 3.37 \\
Examining trade-offs among alternatives & 3.33 \\
Hazardous, chemical, radioactive, and mixed-waste management & 3.29 \\
\hline
\end{tabular}

a Skills with the 10 highest scores.

b 4-point scale: 1 = irrelevant for task; 2 = minimum level of knowledge needed to perform task; $3=$ skill needed to perform task well; and $4=$ critical - one cannot perform this task without knowledge or skill in this area.

\section{TABLE B.2 Most Critical Skills ${ }^{\mathrm{a}}$ Needed to Review Documents}

\begin{tabular}{lc}
\hline \multicolumn{1}{c}{ Skills } & Score ${ }^{\mathrm{b}}$ \\
\hline & \\
EM's mission, policies, management plans, procedures, priorities, goals, and objectives & 3.53 \\
RCRA/CERCLA process & 3.29 \\
Schedule constraints & 3.28 \\
Writing succinctly and clearly & 3.26 \\
Keeping abreast of current developments & 3.26 \\
Time management & 3.26 \\
Examining trade-offs among alternatives & 3.22 \\
Organizing information and presenting cogent arguments & 3.21 \\
Safety and health & 3.18 \\
Assessing strengths and weaknesses of alternatives & 3.17 \\
Organizing/categorizing & 3.16 \\
Human health impacts & 3.12 \\
Radiation effects & 3.12 \\
Hazardous, chemical, radioactive, and mixed-waste management & 3.12 \\
\hline & \\
a Skills with the 10 highest scores. & \\
b 4-point scale: 1 = irrelevant for task; 2 = minimum level of knowledge needed to perform task; $3=$ \\
skill needed to perform task well; and 4 = critical - one cannot perform this task without knowledge \\
or skill in this area.
\end{tabular}


TABLE B.3 Most Critical Skills ${ }^{\mathrm{a}}$ Needed to Coordinate Document Reviews

\begin{tabular}{lc}
\multicolumn{1}{c}{ Skills } & Score $^{\mathrm{b}}$ \\
\hline & 3.47 \\
Providing clear guidance and direction to accomplish tasks & 3.29 \\
EM's mission, policies, management plans, procedures, priorities, goals, and objectives & 3.28 \\
Organizing/categorizing & 3.22 \\
Interpersonal facility (e.g., public, media, other employees, and special interest groups) & 3.18 \\
Negotiating/conflict resolution & 3.18 \\
DOE management strategies & 3.17 \\
Time management & 3.17 \\
Schedule constraints & 3.11 \\
Writing succinctly and clearly & 3.06 \\
Meeting facilitation/leadership & 3.00 \\
DOE departmental organization & 2.89 \\
Interagency agreements, administration, and process & \\
\hline
\end{tabular}

a Skills with the 10 highest scores.

b 4 -point scale: $1=$ irrelevant for task; $2=$ minimum level of knowledge needed to perform task; $3=$ skill needed to perform task well; and $4=$ critical — one cannot perform this task without knowledge or skill in this area.

TABLE B.4 Most Critical Skills ${ }^{\mathrm{a}}$ Needed to Develop Testimony Questions and Answers

\begin{tabular}{lc}
\hline \multicolumn{1}{c}{ Skills } & Score ${ }^{\mathrm{b}}$ \\
\hline EM's mission, policies, management plans, procedures, priorities, goals, and objectives & 3.71 \\
Keeping abreast of current developments & 3.71 \\
Writing succinctly and clearly & 3.50 \\
Organizing information and presenting cogent arguments & 3.44 \\
Time management & 3.29 \\
Identifying relevant material/requirements & 3.25 \\
Organizing/categorizing & 3.24 \\
DOE management strategies & 3.22 \\
Analyzing information & 3.19 \\
Consolidating materials & 3.19 \\
Defining boundaries of a problem & 3.12 \\
Interpersonal facility (e.g., public, media, other employees, and special interest groups) & 3.11 \\
\hline
\end{tabular}

a Skills with the 10 highest scores.

b 4-point scale: 1 = irrelevant for task; 2 = minimum level of knowledge needed to perform task; $3=$ skill needed to perform task well; and $4=$ critical - one cannot perform this task without knowledge or skill in this area. 
TABLE B.5 Most Critical Skills ${ }^{\mathrm{a}}$ Needed to Conduct Briefings/Presentations

Skills

Score ${ }^{b}$

Meeting facilitation/leadership

Public speaking (e.g., public presentations, employee/employer interactions)

Interpersonal facility (e.g., public, media, other employees, and special interest groups)

Organizing information and presenting cogent arguments

EM's mission, policies, management plans, procedures, priorities, goals, and objectives $\quad 3.56$

Organizing/categorizing

Keeping abreast of current developments

DOE management strategies

DOE departmental organization

Writing succinctly and clearly

Anticipating unintended consequences

Negotiating/conflict resolution

a Skills with the 10 highest scores.

b 4-point scale: 1 = irrelevant for task; 2 = minimum level of knowledge needed to perform task; $3=$ skill needed to perform task well; and $4=$ critical - one cannot perform this task without knowledge or skill in this area. 
TABLE B.6 Most Critical Skills ${ }^{\mathrm{a}}$ Needed to Interface with DOE

Skills

Score $^{b}$

Organizing information and presenting cogent arguments

Interpersonal facility (e.g., public, media, other employees, and

3.40 special interest groups)

Negotiating/conflict resolution

Meeting facilitation/leadership

EM's mission, policies, management plans, procedures, priorities,

goals, and objectives

Writing succinctly and clearly

Public speaking (e.g., public presentations, employee/employer interactions)

DOE management strategies

Keeping abreast of current developments $\quad 3.27$

$\begin{array}{ll}\text { Interagency agreements, administration, and process } & 3.20\end{array}$

$\begin{array}{ll}\text { Defining applicable criteria } & 3.08\end{array}$

Providing clear guidance and direction to accomplish tasks $\quad 3.07$

DOE departmental organization $\quad 3.07$

$\begin{array}{ll}\text { Time management } & 3.07\end{array}$

Weighing costs versus benefits $\quad 3.00$

$\begin{array}{ll}\text { Project or program management techniques } & 3.00\end{array}$

$\begin{array}{ll}\text { Schedule constraints } & 3.00\end{array}$

Anticipating unintended consequences $\quad 3.00$

Identifying relevant material/requirements $\quad 3.00$

Defining boundaries of a problem $\quad 2.93$

\begin{tabular}{ll} 
Prioritizing based on criteria & 2.92 \\
\hline
\end{tabular}

a Skills with the 10 highest scores.

b 4-point scale: $1=$ irrelevant for task; 2 = minimum level of knowledge needed to perform task; $3=$ skill needed to perform task well; and $4=$ critical - one cannot perform this task without knowledge or skill in this area. 
TABLE B.7 Most Critical Skills ${ }^{\text {a }}$ Needed to Advise, Assist, and Provide Authoritative Guidance

\begin{tabular}{lc}
\multicolumn{1}{c}{ Skills } & Score \\
\hline & \\
\hline & 3.73 \\
Providing clear guidance and direction to accomplish tasks & 3.58 \\
Orining applicable criteria & 3.40 \\
Writing succinctly and clearly & 3.36 \\
Prioritizing based on criteria & 3.25 \\
Examining trade-offs among alternatives & 3.25 \\
Anticipating unintended consequences & 3.23 \\
Negotiating/conflict resolution & 3.20 \\
DOE management strategies & 3.20 \\
Keeping abreast of current developments & 3.20 \\
Meeting facilitation/leadership & 3.13 \\
EM's mission, policies, management plans, procedures, priorities, & 3.13 \\
goals, and objectives & \\
Identifying relevant material/requirements & 3.08 \\
Reading technical material for a set purpose & 3.08 \\
RCRA/CERCLA process & 3.08 \\
Assessing strengths and weaknesses of alternatives & 3.07 \\
Interagency agreements, administration, and process & 3.07 \\
Defining boundaries of a problem & 3.07 \\
\hline
\end{tabular}

a Skills with the 10 highest scores.

b 4-point scale: 1 = irrelevant for task; 2 = minimum level of knowledge needed to perform task; 3 = skill needed to perform task well; and $4=$ critical - one cannot perform this task without knowledge or skill in this area. 
TABLE B.8 Most Critical Skills ${ }^{\mathrm{a}}$ Needed to Provide Regulatory Analysis and Guidance

Skills Score ${ }^{b}$

RCRA/CERCLA process

Providing clear guidance and direction to accomplish tasks

Interagency agreements, administration, and process

Assessing strengths and weaknesses of alternatives

Organizing information and presenting cogent arguments

EM's mission, policies, management plans, procedures, priorities,

goals, and objectives

Identifying relevant material/requirements

Defining applicable criteria

Examining trade-offs among alternatives

Hazardous, chemical, radioactive, and mixed-waste management

Defining boundaries of a problem

Keeping abreast of current developments

NEPA process

a Skills with the 10 highest scores.

b 4-point scale: $1=$ irrelevant for task; $2=$ minimum level of knowledge needed to perform task; $3=$ skill needed to perform task well; and $4=$ critical - one cannot perform this task without knowledge or skill in this area. 
TABLE B.9 Most Critical Skills ${ }^{\mathrm{a}}$ Needed to Implement Regulations and Policy

\section{Skills}

Score $^{b}$

Comparing existing conditions to pre-set standards

EM's mission, policies, management plans, procedures, priorities, goals, and objectives

Keeping abreast of current developments

Examining trade-offs among alternatives

Prioritizing based on criteria

Project or program management techniques

Schedule constraints

Assessing strengths and weaknesses of alternatives

Anticipating unintended consequences

Interagency agreements, administration, and process

Negotiating/conflict resolution

Writing succinctly and clearly

Providing clear guidance and direction to accomplish tasks

DOE management strategies

RCRA/CERCLA process

Decision analysis

Defining boundaries of a problem

Organizing information and presenting cogent arguments 2.92

Weighing costs versus benefits

Analyzing information

Defining applicable criteria

National consensus standards

Interpersonal facility (e.g., public, media, other employees, and special interest groups)

Identifying relevant material/requirements

a Skills with the 10 highest scores.

b 4-point scale: 1 = irrelevant for task; 2 = minimum level of knowledge needed to perform task; 3 = skill needed to perform task well; and $4=$ critical - one cannot perform this task without knowledge or skill in this area. 
TABLE B.10 Most Critical Skills ${ }^{a}$ Needed to Provide Support to Conduct Permitting/Licensing Activities

\begin{tabular}{lc}
\hline \multicolumn{1}{c}{ Skills } & Score \\
\hline & \\
Comparing existing conditions to pre-set standards & 3.42 \\
RCRAVERCLA process & 3.27 \\
Keeping abreast of current developments & 3.14 \\
Examining trade-offs among alternatives & 3.09 \\
Providing clear guidance and direction to accomplish tasks & 3.07 \\
National consensus standards & 3.00 \\
Prioritizing based on criteria & 3.00 \\
Identifying relevant material/requirements & 3.00 \\
Defining applicable criteria & 3.00 \\
Health and radiological safety impacts and benefits & 3.00 \\
Health physics & 3.00 \\
Hazardous, chemical, radioactive, and mixed-waste management & 3.00 \\
Remediation techniques & 2.91 \\
Human health impacts & 2.91 \\
Radiation effects & 2.91 \\
Safety and health & 2.91 \\
Quality assurance & 2.91 \\
Interagency agreements, administration, and process & 2.86 \\
EM's mission, policies, management plans, procedures, priorities, & 2.86 \\
goals, and objectives & \\
Defining boundaries of a problem & 2.86 \\
Writing succinctly and clearly & 2.85 \\
Anticipating unintended consequences & 2.83 \\
\hline
\end{tabular}

a Skills with the 10 highest scores.

b 4-point scale: $1=$ irrelevant for task; $2=$ minimum level of knowledge needed to perform task; 3 = skill needed to perform task well; and $4=$ critical - one cannot perform this task without knowledge or skill in this area. 
TABLE B.11 Most Critical Skills ${ }^{2}$ Needed to Operate Equipment

Skills Score ${ }^{b}$

Operating and using computer

Using software and applying it to tasks

Touch typing

Understanding principles of database requirements and baseline techniques

2.17

Emergency response procedures

Operating equipment (e.g., testing equipment, respirators)

1.92

Time management

Organizing/categorizing

Anticipating unintended consequences

Identifying relevant material/requirements

1.67

Comparing existing conditions to pre-set standards

Reading technical material for a set purpose

Conducting library research

Keeping abreast of current developments

Prioritizing based on criteria

Delegating tasks

a Skills with the 10 highest scores.

b 4-point scale: 1 = irrelevant for task; 2 = minimum level of knowledge needed to perform task; $3=$ skill needed to perform task well; and $4=$ critical - one cannot perform this task without knowledge or skill in this area. 
TABLE B.12 Most Critical Skills ${ }^{a}$ Needed to Manage Files and Records

Skills

Score $^{b}$

Organizing/categorizing

Consolidating materials

Operating and using computer

Using software and applying it to tasks

Time management

Identifying relevant material/requirements

Organizing information and presenting cogent arguments

Touch typing

Defining applicable criteria

Writing succinctly and clearly

Conducting library research

Delegating tasks

Speed reading

Emergency response procedures

Understanding principles of database requirements and baseline techniques

a Skills with the 10 highest scores.

b 4-point scale: 1 = irrelevant for task; 2 = minimum level of knowledge needed to perform task; 3 = skill needed to perform task well; and $4=$ critical - one cannot perform this task without knowledge or skill in this area. 
TABLE B.13 Most Critical Skills ${ }^{\mathrm{a}}$ Needed to Track Correspondence

\begin{tabular}{|c|c|}
\hline Skills & Score ${ }^{b}$ \\
\hline Operating and using computer & 3.42 \\
\hline Organizing/categorizing & 2.42 \\
\hline Using software and applying it to tasks & 2.42 \\
\hline Touch typing & 2.33 \\
\hline Time management & 2.18 \\
\hline Understanding principles of database requirements and baseline techniques & 2.08 \\
\hline Schedule constraints & 2.00 \\
\hline Writing succinctly and clearly & 1.92 \\
\hline Organizing information and presenting cogent arguments & 1.83 \\
\hline Identifying relevant material/requirements & 1.75 \\
\hline Defining applicable criteria & 1.75 \\
\hline Consolidating materials & 1.75 \\
\hline Delegating authority and responsibility & 1.67 \\
\hline Delegating tasks & 1.67 \\
\hline Emergency response procedures & 1.67 \\
\hline
\end{tabular}

a Skills with the 10 highest scores.

b 4-point scale: 1 = irrelevant for task; $2=$ minimum level of knowledge needed to perform task; $3=$ skill needed to perform task well; and $4=$ critical - one cannot perform this task without knowledge or skill in this area. 
TABLE B.14 Most Critical Skills ${ }^{\mathrm{a}}$ Needed to Perform Travel Administration Functions

\section{Skills}

Score $^{\mathrm{b}}$

Operating and using computer

Organizing/categorizing

Touch typing

Writing succinctly and clearly

Time management

Schedule constraints

Using software and applying it to tasks

Defining applicable criteria

Providing clear guidance and direction to accomplish tasks

Keeping abreast of current developments

Anticipating unintended consequences

Identifying relevant material/requirements

Interpersonal facility (e.g., public, media, other employees, and special interest groups)

Negotiating/conflict resolution

EM's mission, policies, management plans, procedures, priorities, goals, and objectives

Decision analysis

Consolidating materials

Defining boundaries of a problem

Weighing costs versus benefits

Comparing existing conditions to pre-set standards

a Skills with the 10 highest scores.

b 4-point scale: 1 = irrelevant for task; $2=$ minimum level of knowledge needed to perform task; $3=$ skill needed to perform task well; and $4=$ critical - one cannot perform this task without knowledge or skill in this area. 
TABLE B.15 Most Critical Skills ${ }^{\mathrm{a}}$ Needed to Participate in Personnel Training and Development

\section{Skills}

Score ${ }^{b}$

EM's mission, policies, management plans, procedures, priorities, goals, and objectives

Keeping abreast of current developments

Identifying relevant material/requirements

DOE departmental organization

Public speaking (e.g., public presentations, employee/employer interactions)

Equal Opportunity program plans

Defining applicable criteria

Interpersonal facility (e.g., public, media, other employees, and special interest groups)

Project or program management techniques

Assessing strengths and weaknesses of alternatives

Examining trade-offs among alternatives

Providing clear guidance and direction to accomplish tasks

DOE management strategies

Organizing/categorizing

Analyzing information

Writing succinctly and clearly

Meeting facilitation/leadership

Facility, site, operations and processes

Comparing existing conditions to pre-set standards

Defining boundaries of a problem

Time management

Schedule constraints

Planning, programming, funding and acquisition management

Delegating authority and responsibility

Anticipating unintended consequences

Negotiating/conflict resolution

Contractor organization and procedures

Principles of DOE budget formulations, budget execution and cost accounting, and the procedures involved

Reading technical material for a set purpose

a Skills with the 10 highest scores.

b 4-point scale: 1 = irrelevant for task; 2 = minimum level of knowledge needed to perform task; 3 = skill needed to perform task well; and $4=$ critical - one cannot perform this task without knowledge or skill in this area. 
TABLE B.16 Most Critical Skills ${ }^{\mathrm{a}}$ Needed to Oversee

Data/Information Management

\section{Skills} Score ${ }^{b}$

Operating and using computer

Organizing/categorizing

Using software and applying it to tasks

Understanding principles of database requirements and

baseline techniques

Defining applicable criteria

2.58

Keeping abreast of current developments

Identifying relevant material/requirements

Quality assurance

2.42

Writing succinctly and clearly

2.33

Organizing information and presenting cogent arguments $\quad 2.25$

Comparing existing conditions to pre-set standards 2.25

Time management $\quad 2.18$

EM's mission, policies, management plans, procedures, $\quad 2.17$

priorities, goals, and objectives

Decision analysis

Anticipating unintended consequences $\quad 2.17$

Delegating tasks

Emergency response procedures $\quad 2.17$

Touch typing $\quad 2.08$

Schedule constraints $\quad 2.08$

Consolidating materials $\quad 2.08$

a Skills with the 10 highest scores.

b 4-point scale: 1 = irrelevant for task; $2=$ minimum level of knowledge needed to perform task; $3=$ skill needed to perform task well; and $4=$ critical - one cannot perform this task without knowledge or skill in this area. 


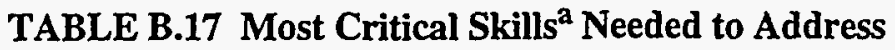

Personnel/Staffing Issues

\section{Skills}

EM's mission, policies, management plans, procedures, priorities, goals, and objectives

Negotiating/conflict resolution

DOE departmental organization

Assessing strengths and weaknesses of alternatives

Anticipating unintended consequences

3.00

Writing succinctly and clearly

Keeping abreast of current developments

Equal Opportunity program plans

2.92

2.83

Organizing information and presenting cogent arguments

DOE management strategies

2.83

Analyzing information

2.82

Organizing/categorizing

2.75

2.75

2.75

2.75

2.75

2.75

2.67

Public speaking (e.g., public presentations, employee/employer

Delegating authority and responsibility

Interpersonal facility (e.g., public, media, other employees, and special interest groups)

$\begin{array}{lr}\text { Project or program management techniques } & 2.58\end{array}$

Delegating tasks $\quad 2.58$

Defining applicable criteria $\quad 2.58$

Defining boundaries of a problem $\quad 2.50$

Weighing costs versus benefits $\quad 2.50$

Planning, programming, funding and acquisition management $\quad 2.42$

a Skills with the 10 highest scores.

b 4-point scale: 1 = irrelevant for task; 2 = minimum level of knowledge needed to perform task; 3 = skill needed to perform task well; and $4=$ critical - one cannot perform this task without knowledge or skill in this area. 
TABLE B.18 Most Critical Skills ${ }^{\mathrm{a}}$ Needed to Track and Verify Corrective Actions

\section{Skills}

Score $^{b}$

Facility, site, operations and processes

Identifying relevant material/requirements

Keeping abreast of current developments

Comparing existing conditions to pre-set standards

Interagency agreements, administration, and process

2.82

Organizing/categorizing

2.75

2.73

EM's mission, policies, management plans, procedures, priorities, goals, and objectives

DOE departmental organization

Defining boundaries of a problem

Schedule constraints

2.67

2.64

2.64

2.64

2.58

2.58

2.50

2.50

2.50

2.45

2.45

Decision analysis

a Skills with the 10 highest scores.

b 4-point scale: 1 = irrelevant for task; 2 = minimum level of knowledge needed to perform task; 3 = skill needed to perform task well; and 4 = critical - one cannot perform this task without knowledge or skill in this area. 
TABLE B.19 Most Critical Skills ${ }^{\text {a }}$ Needed to Oversee Surveillance/ Monitoring/Inspection Activities

Skills

Score ${ }^{b}$

Facility, site, operations and processes

Comparing existing conditions to pre-set standards

Keeping abreast of current developments

Providing clear guidance and direction to accomplish tasks

EM's mission, policies, management plans, procedures, priorities, goals, and objectives

Defining boundaries of a problem

$\begin{array}{lr}\text { Project or program management techniques } & 3.00\end{array}$

Anticipating unintended consequences $\quad 3.00$

$\begin{array}{ll}\text { Prioritizing based on criteria } & 3.00\end{array}$

Identifying relevant material/requirements $\quad 3.00$

Examining trade-offs among alternatives $\quad 3.00$

Hazardous, chemical, radioactive, and mixed-waste management $\quad 3.00$

Quality assurance $\quad 3.00$

DOE management strategies $\quad 2.91$

Assessing strengths and weaknesses of alternatives 2.91

Defining applicable criteria $\quad 2.91$

Remediation techniques $\quad 2.91$

RCRA/CERCLA process $\quad 2.91$

Meeting facilitation/leadership $\quad 2.82$

Organizing/categorizing $\quad 2.82$

Waste treatment/disposal technologies $\quad 2.82$

Human health impacts $\quad 2.82$

Radiation effects $\quad 2.82$

Health and radiological safety impacts and benefits $\quad 2.82$

Health physics $\quad 2.82$

Safety and health $\quad 2.82$

Decision analysis $\quad 2.73$

Schedule constraints $\quad 2.73$

Supervising staff $\quad 2.73$

Delegating tasks $\quad 2.73$

D\&D techniques $\quad 2.73$

Writing succinctly and clearly $\quad 2.64$

DOE departmental organization $\quad 2.64$ 
TABLE B.19 (Cont.)

Skills

Score $^{b}$

Conducting analyses involving complex variables

Delegating authority and responsibility

Analyzing information

Valid engineering solutions

Reading technical material for a set purpose

Packaging issues

Emergency response procedures

Organizing information and presenting cogent arguments

Interpersonal facility (e.g., public, media, other employees, and special interest groups)

Proper engineering/technical standards

Transportation issues

a Skills with the 10 highest scores.

b 4-point scale: 1 = irrelevant for task; $2=$ minimum level of knowledge needed to perform task; $3=$ skill needed to perform task well; and $4=$ critical - one cannot perform this task without knowledge or skill in this area. 
TABLE B.20 Most Critical Skills ${ }^{a}$ Needed to Investigate Complaints, Allegations, and Incidents

\section{Skills}

Identifying relevant material/requirements

Defining boundaries of a problem

Facility, site, operations and processes

Analyzing information

Anticipating unintended consequences

Comparing existing conditions to pre-set standards

Defining applicable criteria

Keeping abreast of current developments

Organizing/categorizing

Reading technical material for a set purpose

Negotiating/conflict resolution

Providing clear guidance and direction to accomplish tasks

Assessing strengths and weaknesses of alternatives

Organizing information and presenting cogent arguments

Interpersonal facility (e.g., public, media, other employees, and special interest groups)

Decision analysis

Examining trade-offs among alternatives

EM's mission, policies, management plans, procedures, priorities, goals, and objectives

Prioritizing based on criteria

Quality assurance
Score $^{\mathrm{b}}$

3.55

3.50

3.40

3.36

3.36

3.27

3.27

3.20

3.20

3.10

3.09

3.09

3.09

3.00

3.00

3.00

3.00

2.91

2.91

2.91

a Skills with the 10 highest scores.

b 4-point scale: 1 = irrelevant for task; 2 = minimum level of knowledge needed to perform task; $3=$ skill needed to perform task well; and $4=$ critical - one cannot perform this task without knowledge or skill in this area. 
TABLE B.21 Most Critical Skills ${ }^{\mathrm{a}}$ Needed to Provide Analyses to Determine Effects of Remediation Projects

\begin{tabular}{lc}
\hline \multicolumn{1}{c}{ Skills } & Score \\
\hline & \\
Analyzing information & 3.50 \\
Remediation techniques & 3.50 \\
Decision analysis & 3.42 \\
Assessing strengths and weaknesses of alternatives & 3.40 \\
Organizing information and presenting cogent arguments & 3.33 \\
RCRA/CERCLA process & 3.27 \\
Writing succinctly and clearly & 3.25 \\
Identifying relevant material/requirements & 3.20 \\
Defining applicable criteria & 3.20 \\
Examining trade-offs among alternatives & 3.20 \\
EM's mission, policies, management plans, procedures, & 3.17 \\
priorities, goals, and objectives & \\
Facility, site, operations and processes & 3.17 \\
Keeping abreast of current developments & 3.17 \\
Prioritizing based on criteria & 3.11 \\
Weighing costs versus benefits & 3.10 \\
Valid engineering solutions & 3.10 \\
Waste treatment/disposal technologies & 3.10 \\
\hline
\end{tabular}

a Skills with the 10 highest scores.

b 4-point scale: 1 = irrelevant for task; 2 = minimum level of knowledge needed to perform task; $3=$ skill needed to perform task well; and $4=$ critical - one cannot perform this task without knowledge or skill in this area. 


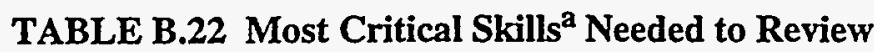
Remediation Projects and Their Progress

\begin{tabular}{ll}
\hline \multicolumn{1}{c}{ Skills } & Score $\mathrm{b}^{\mathrm{b}}$ \\
\hline & \\
Remediation techniques & 3.60 \\
RCRA/CERCLA process & 3.45 \\
Prioritizing based on criteria & 3.33 \\
Identifying relevant material/requirements & 3.30 \\
EM's mission, policies, management plans, procedures, & 3.27 \\
priorities, goals, and objectives & \\
Defining applicable criteria & 3.20 \\
Proper engineering/technical standards & 3.20 \\
Waste treatment/disposal technologies & 3.20 \\
Organizing/categorizing & 3.18 \\
Comparing existing conditions to pre-set standards & 3.10 \\
Valid engineering solutions & 3.10 \\
Interagency agreements, administration, and process & 3.09 \\
Decision analysis & 3.00 \\
Facility, site, operations and processes & 3.00 \\
Defining boundaries of a problem & 3.00 \\
Project or program management techniques & 3.00 \\
Schedule constraints & 3.00 \\
Examining trade-offs among alternatives & 3.00 \\
Human health impacts & 3.00 \\
Radiation effects & 3.00 \\
Health and radiological safety impacts and benefits & 3.00 \\
\hline
\end{tabular}

a Skills with the 10 highest scores.

b 4-point scale: 1 = irrelevant for task; 2 = minimum level of knowledge needed to perform task; $3=$ skill needed to perform task well; and $4=$ critical - one cannot perform this task without knowledge or skill in this area. 
TABLE B.23 Most Critical Skills ${ }^{\mathrm{a}}$ Needed to Recommend Actions to Correct Remedial Problems

\section{Skills} Score $^{\mathrm{b}}$

Remediation techniques

Assessing strengths and weaknesses of alternatives

Analyzing information

Providing clear guidance and direction to accomplish tasks

EM's mission, policies, management plans, procedures, priorities, goals, and objectives

RCRA/CERCLA process

Organizing information and presenting cogent arguments

Planning, programming, funding and acquisition management

Anticipating unintended consequences

Keeping abreast of current developments

Defining boundaries of a problem

Defining applicable criteria

Examining trade-offs among alternatives

a Skills with the 10 highest scores.

b 4-point scale: 1 = irrelevant for task; 2 = minimum level of knowledge needed to perform task; 3 = skill needed to perform task well; and $4=$ critical - one cannot perform this task without knowledge or skill in this area. 
TABLE B.24 Most Critical Skills ${ }^{\mathrm{a}}$ Needed to Identify and Document Deficiencies

\section{Skills}

Score ${ }^{b}$

Interagency agreements, administration, and process

Identifying relevant material/requirements

Analyzing information

Defining applicable criteria

Examining trade-offs among alternatives

EM's mission, policies, management plans, procedures,

Organizing/categorizing

Assessing strengths and weaknesses of alternatives

Comparing existing conditions to pre-set standards

3.10

Decision analysis

Providing clear guidance and direction to accomplish tasks

Defining boundaries of a problem

Weighing costs versus benefits

Project or program management techniques $\quad 3.00$

Anticipating unintended consequences $\quad 3.00$

Writing succinctly and clearly . $\quad 2.91$

Keeping abreast of current developments $\quad 2.91$

RCRA/CERCLA process $\quad 2.91$

Schedule constraints $\quad 2.90$

Prioritizing based on criteria $\quad 2.90$

Reading technical material for a set purpose $\quad 2.90$

Remediation techniques $\quad 2.90$

Organizing information and presenting cogent arguments $\quad 2.82$

a Skills with the 10 highest scores.

b 4-point scale: $1=$ irrelevant for task; $2=$ minimum level of knowledge needed to perform task; $3=$ skill needed to perform task well; and $4=$ critical - one cannot perform this task without knowledge or skill in this area. 
TABLE B.25 Most Critical Skills ${ }^{\mathrm{a}}$ Needed to Set Schedules

\begin{tabular}{lc}
\hline \multicolumn{1}{c}{ Skills } & Score $^{\mathrm{b}}$ \\
\hline Planning, programming, funding and acquisition management & 3.73 \\
Weighing costs versus benefits & 3.18 \\
Conducting analyses involving complex variables & 3.10 \\
Principles of DOE budget formulations, budget execution and cost & 3.09 \\
accounting, and the procedures involved & \\
EM's mission, policies, management plans, procedures, priorities, & 3.08 \\
goals, and objectives & 3.00 \\
Operating equipment (e.g., testing equipment, respirators) & 3.00 \\
Comparing existing conditions to pre-set standards & 3.00 \\
Identifying relevant material/requirements & 2.90 \\
Delegating tasks & 2.83 \\
DOE management strategies & 2.82 \\
Schedule constraints & 2.80 \\
Assessing strengths and weaknesses of alternatives &
\end{tabular}

a Skills with the 10 highest scores.

b 4-point scale: 1 = irrelevant for task; 2 = minimum level of knowledge needed to perform task; $3=$ skill needed to perform task well; and $4=$ critical - one cannot perform this task without knowledge or skill in this area. 
TABLE B.26 Most Critical Skills ${ }^{\mathrm{a}}$ Needed to Identify and Conduct Facility Acceptance

\section{Skills}

Score $^{b}$

EM's mission, policies, management plans, procedures, priorities, goals, and objectives

Comparing existing conditions to pre-set standards

Providing clear guidance and direction to accomplish tasks

3.08

Facility, site, operations and processes

Writing succinctly and clearly

3.00

Identifying relevant material/requirements

3.00

DOE management strategies

Analyzing information

Anticipating unintended consequences

Prioritizing based on criteria

Examining trade-offs among alternatives

Keeping abreast of current developments

Planning, programming, funding and acquisition management $\quad 2.83$

Defining applicable criteria

Organizing information and presenting cogent arguments $\quad 2.75$

$\begin{array}{ll}\text { Negotiating/conflict resolution } & 2.75\end{array}$

DOE departmental organization $\quad 2.75$

Decision analysis $\quad 2.75$

Organizing/categorizing $\quad 2.75$

D\&D techniques $\quad 2.73$

RCRA/CERCLA process $\quad 2.73$

a Skills with the 10 highest scores.

b 4-point scale: 1 = irrelevant for task; 2 = minimum level of knowledge needed to perform task; $3=$ skill needed to perform task well; and $4=$ critical - one cannot perform this task without knowledge or skill in this area. 
TABLE B.27 Most Critical Skills ${ }^{a}$ Needed to Oversee Assessments for Cleanup

Skills

RCRA/CERCLA process

Providing clear guidance and direction to accomplish tasks

Remediation techniques

Interagency agreements, administration, and process

Defining applicable criteria

Writing succinctly and clearly

Identifying relevant material/requirements

Examining trade-offs among alternatives

Organizing information and presenting cogent arguments

EM's mission, policies, management plans, procedures, priorities, goals, and objectives

Keeping abreast of current developments

Supervising staff

Anticipating unintended consequences

Prioritizing based on criteria

Proper engineering/technical standards

DOE management strategies

Facility, site, operations and processes

Schedule constraints

Planning, programming, funding and acquisition management

Delegating authority and responsibility

Comparing existing conditions to pre-set standards

Human health impacts

Safety and health

NEPA process

Environmental sciences
Score ${ }^{b}$

a Skills with the 10 highest scores.

b 4-point scale: 1 = irrelevant for task; $2=$ minimum level of knowledge needed to perform task; $3=$ skill needed to perform task well; and $4=$ critical - one cannot perform this task without knowledge or skill in this area. 
TABLE B.28 Most Critical Skills ${ }^{a}$ Needed to Oversee Environmental Restoration Activities

\section{Skills}

Score $^{\mathrm{b}}$

RCRA/CERCLA process

Interagency agreements, administration, and process

Remediation techniques

Organizing information and presenting cogent arguments

Providing clear guidance and direction to accomplish tasks

Writing succinctly and clearly

EM's mission, policies, management plans, procedures, priorities, goals, and objectives

Keeping abreast of current developments

Anticipating unintended consequences

Defining applicable criteria

Examining trade-offs among alternatives

DOE management strategies

Identifying relevant material/requirements

Proper engineering/technical standards

Human health impacts

Environmental sciences

Facility, site, operations and processes

Planning, programming, funding and acquisition management

a Skills with the 10 highest scores.

b 4-point scale: 1 = irrelevant for task; 2 = minimum level of knowledge needed to perform task; 3 = skill needed to perform task well; and $4=$ critical - one cannot perform this task without knowledge or skill in this area. 
TABLE B.29 Most Critical Skills ${ }^{\mathrm{a}}$ Needed to Oversee

Waste Management Activities

\section{Skills}

Score $^{b}$

RCRA/CERCLA process

Waste treatment/disposal technologies

Providing clear guidance and direction to accomplish tasks

EM's mission, policies, management plans, procedures, priorities, goals, and

objectives

Keeping abreast of current developments

Interagency agreements, administration, and process

3.00

Organizing information and presenting cogent arguments

3.00

DOE management strategies

3.00

Facility, site, operations and processes $\quad 3.00$

Defining applicable criteria

Examining trade-offs among alternatives

Proper engineering/technical standards

3.00

Human health impacts

Packaging issues

3.00

Hazardous, chemical, radioactive, and mixed-waste management 3.00

Writing succinctly and clearly 2.92

Decision analysis $\quad 2.92$

Organizing/categorizing $\quad 2.92$

Planning, programming, funding and acquisition management 2.92

Comparing existing conditions to pre-set standards 2.91

$\begin{array}{ll}\text { Prioritizing based on criteria } & 2.91\end{array}$

Identifying relevant material/requirements $\quad 2.91$

Valid engineering solutions $\quad 2.91$

Remediation techniques $\quad 2.91$

Safety and health $\quad 2.91$

Transportation issues $\quad 2.91$

Environmental sciences $\quad 2.91$

Negotiating/conflict resolution $\quad 2.83$

Meeting facilitation/leadership $\quad 2.83$

Schedule constraints $\quad 2.83$

Supervising staff $\quad 2.83$

Analyzing information $\quad 2.83$

Assessing strengths and weaknesses of alternatives $\quad 2.82$

Anticipating unintended consequences $\quad 2.82$

Delegating tasks $\quad 2.82$

Radiation effects $\quad 2.82$ 
TABLE B.29 (Cont.)

Skills

Score $^{b}$

Health physics

Chemistry

DOE departmental organization

Defining boundaries of a problem

2.75

a Skills with the 10 highest scores.

b 4-point scale: $1=$ irrelevant for task; $2=$ minimum level of knowledge needed to perform task; $3=$ skill needed to perform task well; and $4=$ critical - one cannot perform this task without knowledge or skill in this area. 
TABLE B.30 Most Critical Skills ${ }^{\mathrm{a}}$ Needed to Oversee Toxic and Hazardous Management Activities

Skills

Score $^{b}$

RCRA/CERCLA process

Providing clear guidance and direction to accomplish tasks

EM's mission, policies, management plans, procedures, priorities, goals, and objectives

Waste treatment/disposal technologies

DOE management strategies

Facility, site, operations and processes

Keeping abreast of current developments

Defining applicable criteria

Examining trade-offs among alternatives

Proper engineering/technical standards

Human health impacts

Hazardous, chemical, radioactive, and mixed-waste management

Organizing information and presenting cogent arguments

Writing succinctly and clearly

Decision analysis

Planning, programming, funding and acquisition management

Analyzing information

Safety and health

Packaging issues

Environmental sciences

Prioritizing based on criteria

Identifying relevant material/requirements

Interagency agreements, administration, and process

Meeting facilitation/leadership

DOE departmental organization

Organizing/categorizing

Schedule constraints

Supervising staff

Delegating tasks

Valid engineering solutions

Remediation techniques

Radiation effects

Health physics 
TABLE B.30 (Cont.)

Skills

Score $^{b}$

Transportation issues

Assessing strengths and weaknesses of alternatives

Anticipating unintended consequences

2.80

Comparing existing conditions to pre-set standards

2.80

National consensus standards

2.73

Delegating authority and responsibility

2.73

Geomapping, remote sensing techniques

2.73

Health and radiological safety impacts and benefits $\quad 2.73$

Chemistry

2.73

Land use

NEPA process

Emergency response procedures $\quad 2.73$

Negotiating/conflict resolution $\quad 2.64$

Defining boundaries of a problem $\quad 2.64$

Understanding principles of database requirements and baseline 2.64 techniques

Reading technical material for a set purpose $\quad 2.64$

Consolidating materials $\quad 2.64$

Natural resources $\quad 2.64$

Soils, and minerals $\quad 2.64$

Quality assurance $\quad 2.64$

Public speaking (e.g., public presentations, employee/employer $\quad 2.55$ interactions)

Physical sciences $\quad 2.55$

Geohydrology $\quad 2.55$

Aquatic resources $\quad 2.55$

a Skills with the 10 highest scores.

b 4-point scale: $1=$ irrelevant for task; 2 = minimum level of knowledge needed to perform task; $3=$ skill needed to perform task well; and $4=$ critical - one cannot perform this task without knowledge or skill in this area. 
TABLE B.31 Most Critical Skills ${ }^{\mathrm{a}}$ Needed to Oversee

Water Quality Activities

Skills

Project or program management techniques

Planning, programming, funding and acquisition management

Principles of DOE budget formulations, budget execution and cost accounting, and the procedures involved

Schedule constraints

EM's mission, policies, management plans, procedures, priorities, goals, and objectives

Comparing existing conditions to pre-set standards

Aquatic resources

Identifying relevant material/requirements

Environmental sciences

Keeping abreast of current developments

Assessing strengths and weaknesses of alternatives

Anticipating unintended consequences

Defining applicable criteria

Examining trade-offs among alternatives

Proper engineering/technical standards

Physical sciences

Hazardous, chemical, radioactive, and mixed-waste management

Natural resources

Emergency response procedures

DOE management strategies

Defining boundaries of a problem

Waste treatment/disposal technologies

Biology, zoology, and ecology

Chemistry

Land use
Score ${ }^{b}$

a Skills with the 10 highest scores.

b 4-point scale: 1 = irrelevant for task; 2 = minimum level of knowledge needed to perform task; $3=$ skill needed to perform task well; and $4=$ critical - one cannot perform this task without knowledge or skill in this area. 
TABLE B.32 Most Critical Skills ${ }^{\mathrm{a}}$ Needed to Oversee Air Quality Activities

\begin{tabular}{ll}
\hline \multicolumn{1}{c}{ Skills } & Score \\
\hline & \\
\hline Project or program management techniques & 3.50 \\
Schedule constraints & 3.50 \\
Planning, programming, funding and acquisition management & 3.25 \\
Analyzing information & 3.25 \\
EM's mission, policies, management plans, procedures, priorities, & 3.20 \\
goals, and objectives & \\
Comparing existing conditions to pre-set standards & 3.18 \\
Identifying relevant material/requirements & 3.09 \\
Keeping abreast of current developments & 3.00 \\
Principles of DOE budget formulations, budget execution and cost & 3.00 \\
accounting, and the procedures involved & \\
Assessing strengths and weaknesses of alternatives & 3.00 \\
Anticipating unintended consequences & 3.00 \\
Defining applicable criteria & 3.00 \\
Environmental sciences & 3.00 \\
Examining trade-offs among alternatives & 2.91 \\
Proper engineering/technical standards & 2.91 \\
DOE management strategies & 2.90 \\
Defining boundaries of a problem & 2.90 \\
Chemistry & 2.82 \\
Providing clear guidance and direction to accomplish tasks & 2.80 \\
Decision analysis & 2.80 \\
Facility, site, operations and processes & 2.80 \\
Organizing/categorizing & 2.80 \\
Physical sciences & 2.80 \\
Natural resources & 2.80 \\
Emergency response procedures & 2.80 \\
\hline
\end{tabular}

a Skills with the 10 highest scores.

b 4-point scale: 1 = irrelevant for task; 2 = minimum level of knowledge needed to perform task; 3 = skill needed to perform task well; and $4=$ critical - one cannot perform this task without knowledge or skill in this area. 
TABLE B.33 Most Critical Skills ${ }^{\mathrm{a}}$ Needed to Oversee Cultural Resources Management Activities

\begin{tabular}{|c|c|}
\hline Skills & Score $^{b}$ \\
\hline Schedule constraints & 3.50 \\
\hline Natural resources & 3.30 \\
\hline Project or program management techniques & 3.25 \\
\hline Planning, programming, funding and acquisition management & 3.25 \\
\hline Cultural diversity issues & 3.20 \\
\hline $\begin{array}{l}\text { EM's mission, policies, management plans, procedures, priorities, } \\
\text { goals, and objectives }\end{array}$ & 3.10 \\
\hline Comparing existing conditions to pre-set standards & 3.10 \\
\hline Identifying relevant material/requirements & 3.10 \\
\hline Land use & 3.10 \\
\hline Defining boundaries of a problem & 3.00 \\
\hline Keeping abreast of current developments & 3.00 \\
\hline Analyzing information & 3.00 \\
\hline Assessing strengths and weaknesses of alternatives & 3.00 \\
\hline Defining applicable criteria & 3.00 \\
\hline DOE management strategies & 2.90 \\
\hline Facility, site, operations and processes & 2.90 \\
\hline Examining trade-offs among alternatives & 2.90 \\
\hline Writing succinctly and clearly & 2.80 \\
\hline Negotiating/conflict resolution & 2.80 \\
\hline Providing clear guidance and direction to accomplish tasks & 2.80 \\
\hline Decision analysis & 2.80 \\
\hline Proper engineering/technical standards & 2.80 \\
\hline Time management & 2.75 \\
\hline $\begin{array}{l}\text { Principles of DOE budget formulations, budget execution and cost } \\
\text { accounting, and the procedures involved }\end{array}$ & 2.75 \\
\hline Delegating authority and responsibility & 2.75 \\
\hline Anticipating unintended consequences & 2.75 \\
\hline Interagency agreements, administration, and process & 2.70 \\
\hline Organizing information and presenting cogent arguments & 2.70 \\
\hline Meeting facilitation/leadership & 2.70 \\
\hline Prioritizing based on criteria & 2.70 \\
\hline Waste treatment/disposal technologies & 2.70 \\
\hline Human health impacts & 2.70 \\
\hline
\end{tabular}

a Skills with the 10 highest scores.

b 4-point scale: 1 = irrelevant for task; 2 = minimum level of knowledge needed to perform task; $3=$ skill needed to perform task well; and $4=$ critical - one cannot perform this task without knowledge or skill in this area. 
TABLE B.34 Most Critical Skills ${ }^{\text {a }}$ Needed to Oversee Radiation Protection Activities

\section{Skills}

Score ${ }^{b}$

Project or program management techniques

Schedule constraints

Planning, programming, funding and acquisition management

Analyzing information

Anticipating unintended consequences

Radiation effects

EM's mission, policies, management plans, procedures, priorities, goals, and objectives

Comparing existing conditions to pre-set standards

Identifying relevant material/requirements

Health physics

Facility, site, operations and processes

Keeping abreast of current developments

Organizing/categorizing

Principles of DOE budget formulations, budget execution and

Assessing strengths and weaknesses of alternatives

Defining applicable criteria

Examining trade-offs among alternatives

National consensus standards

DOE management strategies

Defining boundaries of a problem

Proper engineering/technical standards

Human health impacts

Health and radiological safety impacts and benefits

Safety and health

Hazardous, chemical, radioactive, and mixed-waste management

Environmental sciences

Quality assurance

Providing clear guidance and direction to accomplish tasks

Decision analysis

Waste treatment/disposal technologies 
TABLE B.34 (Cont)

\begin{tabular}{lc}
\hline \multicolumn{1}{c}{ Skills } & Score $^{\mathrm{b}}$ \\
\hline & \\
Delegating authority and responsibility & 2.75 \\
Prioritizing based on criteria & 2.70 \\
Biology, zoology, and ecology & 2.70 \\
Physical sciences & 2.70 \\
Emergency response procedures & 2.70 \\
Writing succinctly and clearly & 2.60 \\
Negotiating/conflict resolution & 2.60 \\
Meeting facilitation/leadership & 2.60 \\
Reading technical material for a set purpose & 2.60 \\
Valid engineering solutions & 2.60 \\
RCRA/CERCLA process & 2.60 \\
\hline
\end{tabular}

a Skills with the 10 highest scores.

b 4-point scale: 1 = irrelevant for task; $2=$ minimum level of knowledge needed to perform task; $3=$ skill needed to perform task well; and $4=$ critical - one cannot perform this task without knowledge or skill in this area. 
TABLE B.35 Most Critical Skills ${ }^{a}$ Needed to Oversee NEPA Activities

\section{Skills}

Score $^{b}$

NEPA process

Schedule constraints

Identifying relevant material/requirements

3.36

Assessing strengths and weaknesses of alternatives

3.33

Anticipating unintended consequences

Keeping abreast of current developments

Defining applicable criteria

Defining boundaries of a problem

Negotiating/conflict resolution

3.20

EM's mission, policies, management plans, procedures,

priorities, goals, and objectives

Organizing/categorizing

Comparing existing conditions to pre-set standards

Examining trade-offs among alternatives

RCRA/CERCLA process

Writing succinctly and clearly

Decision analysis

a Skills with the 10 highest scores.

b 4-point scale: 1 = irrelevant for task; 2 = minimum level of knowledge needed to perform task; 3 = skill needed to perform task well; and $4=$ critical - one cannot perform this task without knowledge or skill in this area. 
TABLE B.36 Most Critical Skills ${ }^{a}$ Needed to Oversee

Decontamination and Decommissioning

\section{Skills}

Score ${ }^{b}$

D\&D techniques

EM's mission, policies, management plans, procedures, priorities, goals, and objectives

Weighing costs versus benefits

Project or program management techniques

Radiation effects

Health and radiological safety impacts and benefits

Safety and health

Schedule constraints

Identifying relevant material/requirements

DOE management strategies

Facility, site, operations and processes

Keeping abreast of current developments

Planning, programming, funding and acquisition management

Defining applicable criteria

Examining trade-offs among alternatives

Health physics

NEPA process

Writing succinctly and clearly

Providing clear guidance and direction to accomplish tasks

Defining boundaries of a problem

Organizing/categorizing

Principles of DOE budget formulations, budget execution and cost accounting, and the procedures involved

Analyzing information

Anticipating unintended consequences

Prioritizing based on criteria

Waste treatment/disposal technologies

Human health impacts

Hazardous, chemical, radioactive, and mixed-waste management

Quality assurance

Assessing strengths and weaknesses of alternatives 
TABLE B.36 (Cont.)

Skills

Score ${ }^{b}$

Remediation techniques

RCRA/CERCLA process

2.91

Organizing information and presenting cogent arguments

Proper engineering/technical standards

Time management

2.82

Emergency response procedures

Interagency agreements, administration, and process

DOE departmental organization

2.73

Negotiating/conflict resolution

Environmental sciences

National consensus standards

Consolidating materials

Transportation issues

Packaging issues

2.64

Physical sciences

a Skills with the 10 highest scores.

b 4-point scale: 1 = irrelevant for task; $2=$ minimum level of knowledge needed to perform task; $3=$ skill needed to perform task well; and $4=$ critical - one cannot perform this task without knowledge or skill in this area. 
TABLE B.37 Most Critical Skills ${ }^{\text {a }}$ Needed to Oversee Quality Assurance and Quality Control

\begin{tabular}{|c|c|}
\hline Skills & Score $^{b}$ \\
\hline Emergency response procedures & 3.50 \\
\hline Comparing existing conditions to pre-set standards & 3.20 \\
\hline Defining applicable criteria & 3.20 \\
\hline Providing clear guidance and direction to accomplish tasks & 3.10 \\
\hline DOE management strategies & 3.10 \\
\hline $\begin{array}{l}\text { EM's mission, policies, management plans, priorities, goals, and } \\
\text { objectives }\end{array}$ & 3.10 \\
\hline Keeping abreast of current developments & 3.10 \\
\hline Project or program management techniques & 3.10 \\
\hline Identifying relevant material/requirements & 3.10 \\
\hline Writing succinctly and clearly & 3.00 \\
\hline Defining boundaries of a problem & 3.00 \\
\hline Organizing/categorizing & 3.00 \\
\hline Weighing costs versus benefits & 3.00 \\
\hline Analyzing information & 3.00 \\
\hline Prioritizing based on criteria & 3.00 \\
\hline Facility, site, operations and processes & 2.90 \\
\hline Schedule constraints & 2.90 \\
\hline Planning, programming, funding and acquisition management & 2.90 \\
\hline Assessing strengths and weaknesses of alternatives & 2.90 \\
\hline Examining trade-offs among alternatives & 2.90 \\
\hline Proper engineering/technical standards & 2.90 \\
\hline National consensus standards & 2.80 \\
\hline $\begin{array}{l}\text { Principles of DOE budget formulations, budget execution and } \\
\text { cost accounting, and the procedures involved }\end{array}$ & 2.80 \\
\hline Valid engineering solutions & 2.80 \\
\hline Waste treatment/disposal technologies & 2.80 \\
\hline Radiation effects & 2.80 \\
\hline RCRA/CERCLA process & 2.80 \\
\hline Negotiating/conflict resolution & 2.70 \\
\hline Anticipating unintended consequences & 2.70 \\
\hline Remediation techniques & 2.70 \\
\hline Health and radiological safety impacts and benefits & 2.70 \\
\hline Human health impacts & 2.70 \\
\hline Safety and health & 2.70 \\
\hline
\end{tabular}


TABLE B.37 (Cont.)

Skills

Score $^{b}$

NEPA process

Quality assurance

2.70

Hazardous, chemical, radioactive, and mixed-waste management

2.67

Decision analysis

2.60

Time management

2.60

Delegating tasks

2.60

2.60

Reading technical material for a set purpose

2.60

Consolidating materials

2.60

Health physics

2.60

D\&D techniques

2.50

2.50

DOE departmental organization

2.50

Supervising staff

a Skills with the 10 highest scores.

b 4-point scale: 1 = irrelevant for task; $2=$ minimum level of knowledge needed to perform task; $3=$ skill needed to perform task well; $4=$ critical - one cannot perform this task without knowledge or skill in this area. 
TABLE B.38 Most Critical Skills ${ }^{\mathrm{a}}$ Needed to Oversee Land Use Activities

\section{Skills}

Score ${ }^{b}$

Land use

Examining trade-offs among alternatives

Defining applicable criteria

Providing clear guidance and direction to accomplish tasks

EM's mission, policies, management plans, priorities, and objectives

Identifying relevant material/requirements

Writing succinctly and clearly

DOE management strategies

Defining boundaries of a problem

Keeping abreast of current developments

Interagency agreements, administration, and process

Weighing costs versus benefits

Assessing strengths and weaknesses of alternatives

Prioritizing based on criteria

Negotiating/conflict resolution

Meeting facilitation/leadership

Analyzing information

Anticipating unintended consequences

Organizing information and presenting cogent arguments

Facility, site, operations and processes

Organizing/categorizing

Schedule constraints

RCRA/CERCLA process

Natural resources

NEPA process

Cultural diversity issues

Hazardous, chemical, radioactive, and mixed-waste management

a Skills with the 10 highest scores.

b 4-point scale: 1 = irrelevant for task; 2 = minimum level of knowledge needed to perform task; 3 = skill needed to perform task well; and $4=$ critical - one cannot perform this task without knowledge or skill in this area. 
TABLE B.39 Most Critical Skills ${ }^{\text {a }}$ Needed to Evaluate Alternatives for Cleanup

Skills

Score $^{b}$

Assessing strengths and weaknesses of alternatives

3.78

Examining trade-offs among alternatives

3.70

Weighing costs versus benefits

3.67

Identifying relevant material/requirements

3.50

RCRA/CERCLA process

3.50

Keeping abreast of current developments $\quad 3.44$

Schedule constraints $\quad 3.44$

Prioritizing based on criteria $\quad 3.44$

Remediation techniques $\quad 3.40$

Human health impacts $\quad 3.40$

Writing succinctly and clearly $\quad 3.33$

Providing clear guidance and direction to accomplish tasks 3.33

Organizing/categorizing $\quad 3.33$

Project or program management techniques $\quad 3.33$

Planning, programming, funding and acquisition management 3.33

Analyzing information $\quad 3.33$

Defining applicable criteria $\quad 3.30$

Safety and health $\quad 3.30$

National consensus standards $\quad 3.22$

Interagency agreements, administration, and process $\quad 3.22$

EM's mission, policies, management plans, procedures, priorities, $\quad 3.22$ goals, and objectives

Principles of DOE budget formulations, budget execution and 3.22 cost accounting, and the procedures involved

Anticipating unintended consequences $\quad 3.22$

Comparing existing conditions to pre-set standards $\quad 3.22$

$\begin{array}{ll}\text { Proper engineering/technical standards } & 3.20\end{array}$

Waste treatment/disposal technologies $\quad 3.20$

Health and radiological safety impacts and benefits $\quad 3.20$

a Skills with the 10 highest scores.

b 4-point scale: 1 = irrelevant for task; 2 = minimum level of knowledge needed to perform task; $3=$ skill needed to perform task well; and $4=$ critical - one cannot perform this task without knowledge or skill in this area. 
TABLE B.40 Most Critical Skills ${ }^{\text {a }}$ Needed to Oversee

Program Evaluations

Skills

Score $^{b}$

Project or program management techniques

EM's mission, policies, management plans, procedures,

priorities, goals, and objectives

Principles of DOE budget formulations, budget execution

and cost accounting, and the procedures involved

DOE management strategies

Schedule constraints

Planning, programming, funding and acquisition management

Assessing strengths and weaknesses of alternatives

Identifying relevant material/requirements

3.20

Defining applicable criteria

3.20

Providing clear guidance and direction to accomplish tasks

Defining boundaries of a problem

Keeping abreast of current developments

Organizing/categorizing

Comparing existing conditions to pre-set standards

Examining trade-offs among alternatives

Writing succinctly and clearly

DOE departmental organization

Weighing costs versus benefits

Analyzing information

3.00

Prioritizing based on criteria

3.00

RCRA/CERCLA process

Delegating authority and responsibility

Anticipating unintended consequences

Facility, site, operations and processes

Time management

Conducting analyses involving complex variables

a Skills with the 10 highest scores.

b 4-point scale: $1=$ irrelevant for task; $2=$ minimum level of knowledge needed to perform task; $3=$ skill needed to perform task well; and 4 = critical - one cannot perform this task without knowledge or skill in this area. 
TABLE B.41 Most Critical Skills ${ }^{\mathrm{a}}$ Needed to Manage Contracts

\begin{tabular}{|c|c|}
\hline Skills & Score $^{b}$ \\
\hline Planning, programming, funding and acquisition management & 3.40 \\
\hline EM's mission, policies, management plans, procedures, priorities, goals, and objectives & 3.30 \\
\hline DOE management strategies & 3.20 \\
\hline $\begin{array}{l}\text { Principles of DOE budget formulations, budget execution and cost accounting, and } \\
\text { the procedures involved }\end{array}$ & 3.20 \\
\hline Identifying relevant material/requirements & 3.20 \\
\hline Writing succinctly and clearly & 3.10 \\
\hline Contractor organization and procedures & 3.10 \\
\hline Keeping abreast of current developments & 3.10 \\
\hline Project or program management techniques & 3.10 \\
\hline Supervising staff & 3.10 \\
\hline Defining applicable criteria & 3.10 \\
\hline Negotiating/conflict resolution & 3.00 \\
\hline Providing clear guidance and direction to accomplish tasks & 3.00 \\
\hline Defining boundaries of a problem & 3.00 \\
\hline Organizing/categorizing & 3.00 \\
\hline Schedule constraints & 3.00 \\
\hline Analyzing information & 3.00 \\
\hline Assessing strengths and weaknesses of alternatives & 3.00 \\
\hline DOE departmental organization & 2.90 \\
\hline Anticipating unintended consequences & 2.90 \\
\hline Interpersonal facility (e.g., public, media, other employees, and special interest groups) & 2.80 \\
\hline Weighing costs versus benefits & 2.80 \\
\hline Delegating authority and responsibility & 2.80 \\
\hline Delegating tasks & 2.80 \\
\hline Meeting facilitation/leadership & 2.70 \\
\hline Comparing existing conditions to pre-set standards & 2.70 \\
\hline Prioritizing based on criteria & 2.70 \\
\hline Organizing information and presenting cogent arguments & 2.60 \\
\hline Facility, site, operations and processes & 2.60 \\
\hline Time management & 2.60 \\
\hline Examining trade-offs among alternatives & 2.60 \\
\hline Public speaking (e.g., public presentations, employee/employer interactions) & 2.50 \\
\hline Proper engineering/technical standards & 2.50 \\
\hline
\end{tabular}

a Skills with the 10 highest scores.

b 4-point scale: 1 = irrelevant for task; 2 = minimum level of knowledge needed to perform task; $3=$ skill needed to perform task well; and $4=$ critical - one cannot perform this task without knowledge or skill in this area. 
TABLE B.42 Most Critical Skills ${ }^{\mathrm{a}}$ Needed to Evaluate Contractors

\begin{tabular}{lc}
\hline \multicolumn{1}{c}{ Skills } & Score \\
\hline & \\
\hline Contractor organization and procedures & 3.30 \\
EM's mission, policies, management plans, procedures, priorities, & 3.20 \\
goals, and objectives & \\
Organizing/categorizing & 3.10 \\
Project or program management techniques & 3.10 \\
Schedule constraints & 3.10 \\
Planning, programming, funding and acquisition management & 3.10 \\
Supervising staff & 3.10 \\
Defining applicable criteria & 3.10 \\
Writing succinctly and clearly & 3.00 \\
Providing clear guidance and direction to accomplish tasks & 3.00 \\
Defining boundaries of a problem & 3.00 \\
Keeping abreast of current developments & 3.00 \\
Principles of DOE budget formulations, budget execution and & 3.00 \\
cost accounting, and the procedures involved & \\
Analyzing information & 3.00 \\
Assessing strengths and weaknesses of alternatives & 3.00 \\
Comparing existing conditions to pre-set standards & 3.00 \\
DOE management strategies & 2.90 \\
Organizing information and presenting cogent arguments & 2.80 \\
Interpersonal facility (e.g., public, media, other employees, and & 2.80 \\
special interest groups) & 2.00 \\
Negotiating/conflict resolution & 2.80 \\
Weighing costs versus benefits & 2.60 \\
Delegating authority and responsibility & 2.60 \\
Anticipating unintended consequences & 2.80 \\
Identifying relevant material/requirements & 2.80 \\
Meeting facilitation/leadership & 2.80 \\
Facility, site, operations and processes & 2.80 \\
Prioritizing based on criteria & 2.70 \\
Delegating tasks & 2.70 \\
Examining trade-offs among alternatives & 2.70 \\
DOE departmental organization & 2.70 \\
Decision analysis & 2.70 \\
Time management & 2.60 \\
Reading technical material for a set purpose & \\
\hline & \\
Public speaking (e.g., public presentations, employee/employer interactions) & \\
\hline
\end{tabular}

a Skills with the 10 highest scores.

b 4 -point scale: $1=$ irrelevant for task; $2=$ minimum level of knowledge needed to perform task; 3 = skill needed to perform task well; and $4=$ critical - one cannot perform this task without knowledge or skill in this area. 
TABLE B.43 Most Critical Skills ${ }^{\mathrm{a}}$ Needed to Oversee Independent Verification

\section{Skills}

Score $^{b}$

EM's mission, policies, management plans, priorities, goals, and objectives

Providing clear guidance and direction to accomplish tasks

Defining boundaries of a problem

Organizing/categorizing

Analyzing information

Comparing existing conditions to pre-set standards

Defining applicable criteria

Writing succinctly and clearly

Facility, site, operations and processes

Keeping abreast of current developments

Identifying relevant material/requirements

Negotiating/conflict resolution

DOE management strategies

Contractor organization and procedures

Project or program management techniques

Schedule constraints

Assessing strengths and weaknesses of alternatives

Anticipating unintended consequences

Organizing information and presenting cogent arguments

Supervising staff

Prioritizing based on criteria

Delegating tasks

D\&D techniques

Interpersonal facility (e.g., public, media, other employees, and special interest groups)

Meeting facilitation/leadership

DOE departmental organization

Weighing costs versus benefits

Delegating authority and responsibility

Remediation techniques

Public speaking (e.g. public presentations, employee/employer ctions)

Decision analysis

Time management

Planning, programming, funding and acquisition management

Reading technical material for a set purpose

Examining trade-offs among alternatives 
TABLE B.43 (Cont.)

\begin{tabular}{lc}
\hline \multicolumn{1}{c}{ Skills } & Score \\
\hline Proper engineering/technical standards & 2.60 \\
Valid engineering solutions & 2.60 \\
Waste treatment/disposal technologies & 2.60 \\
Safety and health & 2.60 \\
Principles of DOE budget formulations, budget execution and & 2.50 \\
cost accounting, and the procedures involved & \\
Radiation effects & 2.50 \\
Consolidating materials & 2.40 \\
Health and radiological safety impacts and benefits & 2.40 \\
Health physics & 2.40 \\
\hline
\end{tabular}

a Skills with the 10 highest scores.

b 4-point scale: 1 = irrelevant for task; $2=$ minimum level of knowledge needed to perform task; $3=$ skill needed to perform task well; and 4 = critical - one cannot perform this task without knowledge or skill in this area. 
TABLE B.44 Most Critical Skills ${ }^{\mathrm{a}}$ Needed to Identify and

Allocate Resources

Skills

EM's mission, policies, management plans, procedures, priorities, goals, and objectives

Planning, programming, funding and acquisition management

Weighing costs versus benefits

Principles of DOE budget formulations, budget execution and cost accounting, and the procedures involved

Identifying relevant material/requirements

DOE departmental organization

Defining boundaries of a problem

Organizing/categorizing

Facility, site, operations and processes

Keeping abreast of current developments

Project or program management techniques

Defining applicable criteria

DOE management strategies

Schedule constraints

Analyzing information

Anticipating unintended consequences

Examining trade-offs among alternatives

Writing succinctly and clearly

Negotiating/conflict resolution

Providing clear guidance and direction to accomplish tasks

Time management

Delegating authority and responsibility

Assessing strengths and weaknesses of altematives

Prioritizing based on criteria

Contractor organization and procedures

Decision analysis

Interpersonal facility (e.g., public, media, other employees, and special interest groups)

Supervising staff

Delegating tasks

Organizing information and presenting cogent arguments

Public speaking (e.g., public presentations, employee/employer interactions)

Meeting facilitation/leadership

Understanding principles of database requirements and baseline techniques

Consolidating materials
Score ${ }^{b}$

3.30

3.20

3.10

3.10

3.10

3.00

3.00

3.00

2.90

2.90

2.90

2.90

2.80

2.80

2.80

2.80

2.80

2.70

2.70

2.70

2.70

2.70

2.70

2.70

2.60

2.60

2.50

2.50

2.50

2.40

2.40

2.40

2.40

2.40

a Skills with the 10 highest scores.

b 4-point scale: 1 = irrelevant for task; $2=$ minimum level of knowledge needed to perform task; 3 = skill needed to perform task well; 4 = critical — one cannot perform this task without knowledge or skill in this area. 
TABLE B.45 Most Critical Skills ${ }^{\text {a }}$ Needed to Provide Goods and Services

Skills Score $^{\mathrm{b}}$

Planning, programming, funding and acquisition management

Weighing costs versus benefits

Principles of DOE budget formulations, budget execution and cost accounting, and the procedures involved

EM's mission, policies, management plans, procedures, priorities, goals, and objectives

Keeping abreast of current developments

Organizing/categorizing

Schedule constraints

Providing clear guidance and direction to accomplish tasks

Defining boundaries of a problem

Identifying relevant material/requirements

Defining applicable criteria

Writing succinctly and clearly

Facility, site, operations and processes

Prioritizing based on criteria

Negotiating/conflict resolution

DOE departmental organization

Time management

Project or program management techniques

Analyzing information

Anticipating unintended consequences

Examining trade-offs among alternatives

DOE management strategies

Contractor organization and procedures

Understanding principles of database requirements and baseline

Delegating authority and responsibility

Comparing existing conditions to pre-set standards

Meeting facilitation/leadership

Assessing strengths and weaknesses of alternatives

Interpersonal facility (e.g., public, media, other employees, and special interest groups) 


\section{TABLE B.45 (Cont.)}

\begin{tabular}{lc}
\hline \multicolumn{1}{c}{ Skills } & Score $^{\mathrm{b}}$ \\
\hline & \\
Decision analysis & 2.20 \\
Reading technical material for a set purpose & 2.10 \\
Proper engineering/technical standards & 2.10 \\
Valid engineering solutions & 2.10 \\
\hline
\end{tabular}

a Skills with the 10 highest scores.

b 4-point scale: 1 = irrelevant for task; 2 = minimum level of knowledge needed to perform task; 3 = skill needed to perform task well; and $4=$ critical - one cannot perform this task without knowledge or skill in this area. 
TABLE B.46 Most Critical Skills ${ }^{2}$ Needed to Oversee Cost Reviews

\begin{tabular}{ll}
\hline \multicolumn{1}{c}{ Skills } & Score \\
\hline & \\
\hline Weighing costs versus benefits & 3.20 \\
Project or program management techniques & 3.20 \\
EM's mission, policies, management plans, procedures, priorities, goals, & 3.10 \\
and objectives & \\
Planning, programming, funding and acquisition management & 3.10 \\
Identifying relevant material/requirements & 3.10 \\
Examining trade-offs among alternatives & 3.10 \\
Principles of DOE budget formulations, budget execution and & 3.00 \\
cost accounting, and the procedures involved & \\
Providing clear guidance and direction to accomplish tasks & 2.90 \\
Facility, site, operations and processes & 2.90 \\
Defining boundaries of a problem & 2.90 \\
Schedule constraints & 2.90 \\
Analyzing information & 2.90 \\
Prioritizing based on criteria & 2.90 \\
Defining applicable criteria & 2.90 \\
DOE management strategies & 2.80 \\
Decision analysis & 2.80 \\
Keeping abreast of current developments & 2.80 \\
Organizing/categorizing & 2.80 \\
Writing succinctly and clearly & 2.70 \\
Negotiating/conflict resolution & 2.70 \\
Meeting facilitation/leadership & 2.70 \\
Assessing strengths and weaknesses of alternatives & 2.70 \\
Comparing existing conditions to pre-set standards & 2.50 \\
Organizing information and presenting cogent arguments & 2.70 \\
Supervising staff & 2.60 \\
Delegating authority and responsibility & 2.60 \\
Anticipating unintended consequences & 2.60 \\
DOE departmental organization & 2.60 \\
Contractor organization and procedures & 2.50 \\
Reading technical material for a set purpose & 2.50 \\
Consolidating materials & 2.50 \\
Proper engineering/technical standards & \\
Valid engineering solutions & \\
& \\
\hline
\end{tabular}


TABLE B.46 (Cont.)

Skills

Score $^{\mathrm{b}}$

Remediation techniques

2.50

Interpersonal facility (e.g., public, media, other employees, and special interest groups)

Understanding principles of database requirements and baseline techniques

Delegating tasks

D\&D techniques

a Skills with the 10 highest scores.

b 4-point scale: 1 = irrelevant for task; 2 = minimum level of knowledge needed to perform task; $3=$ skill needed to perform task well; and $4=$ critical - one cannot perform this task without knowledge or skill in this area. 
TABLE B.47 Most Critical Skills ${ }^{\mathrm{a}}$ Needed to Formulate, Defend, and Modify Budget

\begin{tabular}{ll}
\hline \multicolumn{1}{c}{ Skills } & Score \\
\hline & \\
\hline & 3.90 \\
Principles of DOE budget formulations, budget execution and & \\
EM's mission, policies, management plans, procedures, priorities, & 3.70 \\
goals, and objectives & \\
Identifying relevant material/requirements & 3.50 \\
Planning, programming, funding and acquisition management & 3.40 \\
Assessing strengths and weaknesses of alternatives & 3.40 \\
Prioritizing based on criteria & 3.40 \\
Examining trade-offs among alternatives & 3.40 \\
Writing succinctly and clearly & 3.30 \\
Providing clear guidance and direction to accomplish tasks & 3.30 \\
DOE management strategies & 3.30 \\
Project or program management techniques & 3.30 \\
Schedule constraints & 3.30 \\
Weighing costs versus benefits & 3.20 \\
Analyzing information & 3.20 \\
Anticipating unintended consequences & 3.20 \\
Defining applicable criteria & 3.20 \\
Organizing information and presenting cogent arguments & 3.10 \\
Interpersonal facility (e.g., public, media, other employees, and & 3.10 \\
special interest groups) & \\
Negotiating/conflict resolution & 3.10 \\
Decision analysis & 3.10 \\
Keeping abreast of current developments & 3.10 \\
Organizing/categorizing & 3.10 \\
Interagency agreements, administration, and process & 3.00 \\
Meeting facilitation/leadership & 3.00 \\
DOE departmental organization & 3.00 \\
Defining boundaries of a problem & 3.00 \\
Comparing existing conditions to pre-set standards & 3.00 \\
Consolidating materials & 2.90 \\
Reading technical material for a set purpose & 2.80 \\
Remediation techniques & 2.80 \\
\hline
\end{tabular}

a Skills with the 10 highest scores.

b 4-point scale: 1 = irrelevant for task; 2 = minimum level of knowledge needed to perform task; $3=$ skill needed to perform task well; and $4=$ critical - one cannot perform this task without knowledge or skill in this area. 
TABLE B.48 Most Critical Skills ${ }^{\mathrm{a}}$ Needed to Develop, Coordinate, and Disseminate Program Management Policies and Procedures

\begin{tabular}{|c|c|}
\hline Skills & Score $^{b}$ \\
\hline Project or program management techniques & 3.60 \\
\hline $\begin{array}{l}\text { EM's mission, policies, management plans, procedures, priorities, } \\
\text { goals, and objectives }\end{array}$ & 3.30 \\
\hline Examining trade-offs among alternatives & 3.20 \\
\hline Providing clear guidance and direction to accomplish tasks & 3.10 \\
\hline DOE management strategies & 3.10 \\
\hline $\begin{array}{l}\text { Principles of DOE budget formulations, budget execution and } \\
\text { cost accounting, and the procedures involved }\end{array}$ & 3.10 \\
\hline Identifying relevant material/requirements & 3.10 \\
\hline Writing succinctly and clearly & 3.00 \\
\hline DOE departmental organization & 3.00 \\
\hline Schedule constraints & 3.00 \\
\hline Planning, programming, funding and acquisition management & 3.00 \\
\hline Analyzing information & 3.00 \\
\hline Assessing strengths and weaknesses of alternatives & 3.00 \\
\hline Comparing existing conditions to pre-set standards & 3.00 \\
\hline Prioritizing based on criteria & 3.00 \\
\hline Defining applicable criteria & 3.00 \\
\hline Decision analysis & 2.90 \\
\hline Organizing/categorizing & 2.90 \\
\hline Delegating authority and responsibility & 2.90 \\
\hline $\begin{array}{l}\text { Interpersonal facility (e.g., public, media, other employees, and } \\
\text { special interest groups) }\end{array}$ & 2.80 \\
\hline Negotiating/conflict resolution & 2.80 \\
\hline Meeting facilitation/leadership & 2.80 \\
\hline Defining boundaries of a problem & 2.80 \\
\hline Keeping abreast of current developments & 2.80 \\
\hline Anticipating unintended consequences & 2.80 \\
\hline Organizing information and presenting cogent arguments & 2.70 \\
\hline Weighing costs versus benefits & 2.70 \\
\hline Interagency agreements, administration, and process & 2.60 \\
\hline Facility, site, operations and processes & 2.60 \\
\hline Supervising staff & 2.60 \\
\hline Reading technical material for a set purpose & 2.60 \\
\hline RCRA/CERCLA process & 2.55 \\
\hline
\end{tabular}

a Skills with the 10 highest scores.

b 4-point scale: 1 = irrelevant for task; 2 = minimum level of knowledge needed to perform task; 3 = skill needed to perform task well; and $4=$ critical - one cannot perform this task without knowledge or skill in this area. 
TABLE B.49 Most Critical Skills ${ }^{2}$ Needed to Establish Cost Schedule and Technical Baselines

\section{Skills}

Score $^{b}$

EM's mission, policies, management plans, procedures, priorities, goals, and objectives

Anticipating unintended consequences

Project or program management techniques

3.30

Planning, programming, funding and acquisition management

DOE management strategies

3.20

Principles of DOE budget formulations, budget execution and

3.10

cost accounting, and the procedures involved

Identifying relevant material/requirements

3.10

Examining trade-offs among alternatives

3.10

Keeping abreast of current developments

3.10

Organizing/categorizing

3.00

Prioritizing based on criteria

3.00

Remediation techniques

3.00

D\&D techniques

3.00

Writing succinctly and clearly

Providing clear guidance and direction to accomplish tasks

Decision analysis

2.90

Defining boundaries of a problem

2.90

Schedule constraints

2.90

Analyzing information

Defining applicable criteria

2.90

Interagency agreements, administration, and process 2.80

Facility, site, operations and processes $\quad 2.80$

Weighing costs versus benefits $\quad 2.80$

Waste treatment/disposal technologies $\quad 2.80$

RCRA/CERCLA process $\quad 2.73$

Organizing information and presenting cogent arguments $\quad 2.70$

Negotiating/conflict resolution $\quad 2.70$

Understanding principles of database requirements and baseline techniques $\quad 2.70$

Assessing strengths and weaknesses of alternatives $\quad 2.70$

Comparing existing conditions to pre-set standards $\quad 2.70$

$\begin{array}{ll}\text { Valid engineering solutions } & 2.70\end{array}$

Hazardous, chemical, radioactive, and mixed-waste management $\quad 2.64$

a Skills with the 10 highest scores.

b 4-point scale: $1=$ irrelevant for task; $2=$ minimum level of knowledge needed to perform task; $3=$ skill needed to perform task well; and $4=$ critical - one cannot perform this task without knowledge or skill in this area. 
TABLE B.50 Most Critical Skills ${ }^{\mathrm{a}}$ Needed to Develop Resource Requirements (Budgets/FTEs)

Skills

Score ${ }^{b}$

Principles of DOE budget formulations, budget execution and

cost accounting, and the procedures involved

Planning, programming, funding and acquisition management

Identifying relevant material/requirements

EM's mission, policies, management plans, procedures, priorities, goals, and objectives

Prioritizing based on criteria

Examining trade-offs among altematives

Writing succinctly and clearly

DOE management strategies

DOE departmental organization

Schedule constraints

Analyzing information

Assessing strengths and weaknesses of alternatives $\quad 3.20$

$\begin{array}{ll}\text { Anticipating unintended consequences } & 3.20\end{array}$

Defining applicable criteria $\quad 3.20$

Interpersonal facility (e.g., public, media, other employees, and $\quad 3.10$ special interest groups)

Providing clear guidance and direction to accomplish tasks $\quad 3.10$

Keeping abreast of current developments $\quad 3.10$

Defining boundaries of a problem $\quad 3.00$

$\begin{array}{ll}\text { Organizing/categorizing } & 3.00\end{array}$

Project or program management techniques $\quad 3.00$

Comparing existing conditions to pre-set standards $\quad 3.00$

Consolidating materials $\quad 3.00$

Interagency agreements, administration, and process $\quad 2.90$

Organizing information and presenting cogent arguments $\quad 2.90$

$\begin{array}{ll}\text { Negotiating/conflict resolution } & 2.80\end{array}$

Decision analysis $\quad 2.80$

Weighing costs versus benefits $\quad 2.80$

Remediation techniques $\quad 2.80$

Public speaking (e.g., public presentations, employee/employer interactions) $\quad 2.70$

$\begin{array}{ll}\text { Meeting facilitation/leadership } & 2.70\end{array}$

$\begin{array}{ll}\text { Supervising staff } & 2.70\end{array}$

Waste treatment/disposal technologies $\quad 2.70$

RCRA/CERCLA process $\quad 2.64$

a Skills with the 10 highest scores.

b 4-point scale: 1 = irrelevant for task; 2 = minimum level of knowledge needed to perform task; 3 = skill needed to perform task well; and 4 = critical — one cannot perform this task without knowledge or skill in this area. 


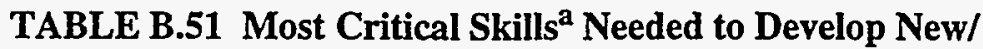
Improve Technologies

Skills

Score ${ }^{\mathrm{b}}$

Keeping abreast of current developments

Assessing strengths and weaknesses of alternatives

3.40

Identifying relevant material/requirements

3.40

Examining trade-offs among alternatives

3.40

Prioritizing based on criteria

3.30

EM's mission, policies, management plans, procedures, priorities, goals, and objectives

Anticipating unintended consequences

Defining applicable criteria

Reading technical material for a set purpose

Remediation techniques

3.20

Defining boundaries of a problem

3.10

Weighing costs versus benefits

3.10

Analyzing information

3.10

Waste treatment/disposal technologies

3.10

DOE management strategies

3.00

Comparing existing conditions to pre-set standards

3.00

Geomapping, remote sensing techniques

3.00

Proper engineering/technical standards

3.00

Valid engineering solutions

3.00

Hazardous, chemical, radioactive, and mixed-waste management

2.91

Writing succinctly and clearly

2.90

Providing clear guidance and direction to accomplish tasks

2.90

DOE departmental organization

2.90

Decision analysis

2.90

Schedule constraints

2.90

Conducting analyses involving complex variables

$D \& D$ techniques

2.82

RCRA/CERCLA process

2.82

Emergency response procedures

2.82

Organizing information and presenting cogent arguments

2.80

Interpersonal facility (e.g., public, media, other employees, and special interest groups)

Facility, site, operations and processes

Organizing/categorizing 
TABLE B.51 (Cont.)

\begin{tabular}{lc}
\hline \multicolumn{1}{c}{ Skills } & Score $^{\mathrm{b}}$ \\
\hline & 2.80 \\
Principles of DOE budget formulations, budget execution and cost & \\
accounting, and the procedures involved & 2.80 \\
Planning, programming, funding and acquisition management & 2.80 \\
Human health impacts &
\end{tabular}

a Skills with the 10 highest scores.

b 4-point scale: 1 = irrelevant for task; 2 = minimum level of knowledge needed to perform task; 3 = skill needed to perform task well; and $4=$ critical - one cannot perform this task without knowledge or skill in this area. 
TABLE B.52 Most Critical Skills ${ }^{\mathrm{a}}$ Needed to Oversee Research, Development, Demonstration, Testing, and

Evaluation Activities

\begin{tabular}{|c|c|}
\hline Skills & Score ${ }^{\mathrm{b}}$ \\
\hline Keeping abreast of current developments & 3.40 \\
\hline Identifying relevant material/requirements & 3.30 \\
\hline Reading technical material for a set purpose & 3.30 \\
\hline $\begin{array}{l}\text { EM's mission, policies, management plans, procedures, priorities, } \\
\text { goals, and objectives }\end{array}$ & 3.20 \\
\hline Assessing strengths and weaknesses of alternatives & 3.20 \\
\hline Prioritizing based on criteria & 3.20 \\
\hline Examining trade-offs among alternatives & 3.20 \\
\hline Anticipating unintended consequences & 3.10 \\
\hline Defining applicable criteria & 3.10 \\
\hline DOE management strategies & 3.00 \\
\hline Organizing/categorizing & 3.00 \\
\hline Analyzing information & 3.00 \\
\hline Proper engineering/technical standards & 3.00 \\
\hline Valid engineering solutions & 3.00 \\
\hline Remediation techniques & 3.00 \\
\hline Organizing information and presenting cogent arguments & 2.90 \\
\hline Writing succinctly and clearly & 2.90 \\
\hline Providing clear guidance and direction to accomplish tasks & 2.90 \\
\hline Defining boundaries of a problem & 2.90 \\
\hline Schedule constraints & 2.90 \\
\hline Comparing existing conditions to pre-set standards & 2.90 \\
\hline Geomapping, remote sensing techniques & 2.90 \\
\hline Waste treatment/disposal technologies & 2.90 \\
\hline Negotiating/conflict resolution & 2.80 \\
\hline DOE departmental organization & 2.80 \\
\hline Decision analysis & 2.80 \\
\hline Facility, site, operations and processes & 2.80 \\
\hline Weighing costs versus benefits & 2.80 \\
\hline Planning, programming, funding and acquisition management & 2.80 \\
\hline Conducting analyses involving complex variables & 2.80 \\
\hline $\begin{array}{l}\text { Interpersonal facility (e.g., public, media, other employees, and } \\
\text { special interest groups) }\end{array}$ & 2.70 \\
\hline Meeting facilitation/leadership & 2.70 \\
\hline Project or program management techniques & 2.70 \\
\hline $\begin{array}{l}\text { Principles of DOE budget formulations, budget execution and } \\
\text { cost accounting, and the procedures involved }\end{array}$ & 2.70 \\
\hline
\end{tabular}




\section{$B-63$}

TABLE B.52 (Cont.)

\begin{tabular}{lc}
\hline \multicolumn{1}{c}{ Skills } & Score $^{\mathrm{b}}$ \\
\hline Delegating tasks & 2.70 \\
Health and radiological safety impacts and benefits & 2.70 \\
Hazardous, chemical, radioactive, and mixed-waste management & 2.64 \\
Human health impacts & 2.60 \\
Radiation effects & 2.60 \\
Safety and health & 2.60 \\
Transportation issues & 2.60 \\
\hline
\end{tabular}

a Skills with the 10 highest scores.

b 4-point scale: $1=$ irrelevant for task; $2=$ minimum level of knowledge needed to perform task; $3=$ skill needed to perform task well; and $4=$ critical - one cannot perform this task without knowledge or skill in this area. 

C-1

APPENDIX C:

SKILLS WITH HIGHEST AND LOWEST AVERAGE SCORES 
C-2

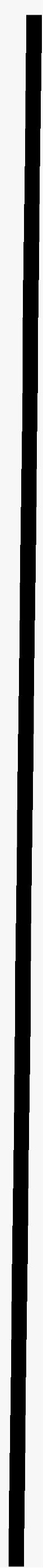


TABLE C.1 10 Highest Average Scores on General Knowledge/Skills

\begin{tabular}{|c|c|c|c|}
\hline General Skill & Average $^{a}$ & $\begin{array}{l}\text { Wide Range } \\
\text { of Abilities }\end{array}$ & $\begin{array}{l}\text { Common } \\
\text { Skills }\end{array}$ \\
\hline Organizing/categorizing & 3.14 & $\checkmark$ & $\checkmark$ \\
\hline Analyzing information & 3.14 & & $\checkmark$ \\
\hline Conducting library research & 3.05 & & \\
\hline Organizing information and presenting cogent arguments & 3.05 & & $\checkmark$ \\
\hline Operating and using computer & 3.05 & & \\
\hline Assessing strengths and weaknesses of alternatives & 3.05 & & $\checkmark$ \\
\hline Prioritizing based on criteria & 3.05 & & $\checkmark$ \\
\hline Writing succinctly and clearly & 2.95 & & $\checkmark$ \\
\hline Providing clear guidance and direction to accomplish tasks & 2.95 & & $\checkmark$ \\
\hline Keeping abreast of current developments & 2.95 & $\checkmark$ & $\checkmark$ \\
\hline Consolidating materials & 2.95 & $\cdot$ & \\
\hline Project or program management techniques & 2.90 & $\checkmark$ & $\checkmark$ \\
\hline Schedule constraints & 2.90 & & $\checkmark$ \\
\hline Examining trade-offs among alternatives & 2.90 & & $\checkmark$ \\
\hline Defining boundaries of a problem & 2.86 & & \\
\hline Weighing costs versus benefits & 2.86 & $\checkmark$ & \\
\hline Reading technical material for a set purpose & 2.85 & $\checkmark$ & \\
\hline Anticipating unintended consequences & 2.81 & & \\
\hline Identifying relevant materials/requirements & 2.81 & & \\
\hline Delegating tasks & 2.81 & & \\
\hline $\begin{array}{l}\text { Public speaking (e.g., public presentations, } \\
\text { employee/employer interactions) }\end{array}$ & 2.80 & $\checkmark$ & \\
\hline Meeting facilitation/leadership & 2.76 & $\checkmark$ & \\
\hline DOE departmental organization & 2.76 & & \\
\hline Comparing existing conditions to preset standards & 2.76 & & \\
\hline Time management & 2.75 & & \\
\hline Defining applicable criteria & 2.75 & & $\checkmark$ \\
\hline
\end{tabular}

a Average score is based on a 4-point scale: $1=$ no/little knowledge; 2 = heard of/familiar with; $3=$ proficient; $4=$ expert. 
TABLE C.2 10 Lowest Average Scores on General Knowledge/Skills

\begin{tabular}{|c|c|c|c|}
\hline General Skill & Average $^{\mathrm{a}}$ & $\begin{array}{c}\text { Wide } \\
\text { Range of } \\
\text { Abilities }\end{array}$ & $\begin{array}{l}\text { Common } \\
\text { Skills }\end{array}$ \\
\hline $\begin{array}{l}\text { Understanding principles of database requirements and } \\
\text { baseline techniques }\end{array}$ & 2.62 & & \\
\hline Decision analysis & 2.60 & & \\
\hline Planning, programming, funding, and acquisition management & 2.52 & & $\checkmark$ \\
\hline Facility, site, operations and processes & 2.43 & $\checkmark$ & \\
\hline DOE management strategies & 2.38 & & $\checkmark$ \\
\hline Conducting analyses involving complex variables & 2.38 & & \\
\hline Operating equipment (e.g., testing equipment, respirators) & 2.14 & $\checkmark$ & \\
\hline Contractor organization and procedures & 2.10 & & \\
\hline Touch typing & 1.95 & $\checkmark$ & \\
\hline Equal Opportunity Program plans & 1.95 & $\checkmark$ & \\
\hline National consensus standards & 1.68 & & \\
\hline Speed reading & 1.67 & $\checkmark$ & \\
\hline
\end{tabular}

.a Average score is based on a 4-point scale: 1 = no/little knowledge; 2 = heard of/familiar with; $3=$ proficient; $4=$ expert. 
TABLE C.3 10 Highest Average Scores on Technical Knowledge

\begin{tabular}{|c|c|c|c|}
\hline General Skill & Average $^{a}$ & $\begin{array}{l}\text { Wide Range } \\
\text { of Abilities }\end{array}$ & $\begin{array}{l}\text { Common } \\
\text { Skills }\end{array}$ \\
\hline RCRA/CERCLA process & 2.95 & $\checkmark$ & $\checkmark$ \\
\hline NEPA process & 2.67 & $\checkmark$ & \\
\hline Environmental sciences & 2.67 & $\checkmark$ & \\
\hline Remediation techniques & 2.62 & $\checkmark$ & $\checkmark$ \\
\hline Physical sciences & 2.57 & $\checkmark$ & \\
\hline $\begin{array}{l}\text { Hazardous, chemical, radioactive, and mixed waste } \\
\text { management }\end{array}$ & 2.57 & $\checkmark$ & \\
\hline Valid engineering solutions & 2.40 & $\checkmark$ & \\
\hline Biology, zoology, and ecology & 2.38 & $\checkmark$ & \\
\hline Waste treatment/disposal technologies & 2.33 & $\checkmark$ & \\
\hline Natural resources & 2.33 & $\checkmark$ & \\
\hline Radiation effects & 2.29 & $\checkmark$ & \\
\hline Safety and health & 2.24 & & \\
\hline Transportation issues & 2.24 & & \\
\hline Chemistry & 2.24 & & \\
\hline Emergency response procedures & 2.24 & $\checkmark$ & \\
\hline Quality assurance & 2.24 & & \\
\hline D\&D techniques & 2.19 & $\checkmark$ & \\
\hline Land use & 2.19 & $\checkmark$ & \\
\hline
\end{tabular}

a Average score is based on a 4-point scale: $1=$ no/little knowledge; $2=$ heard of/familiar with; $3=$ proficient; $4=$ expert. 


\begin{tabular}{lccc}
\hline \multicolumn{1}{c}{ General Skill } & & Wide Range & $\begin{array}{c}\text { Common } \\
\text { Skills }\end{array}$ \\
\hline & Average $^{2}$ & of Abilities & \\
D\&D techniques & 2.19 & $\checkmark$ \\
Land use & 2.19 & \\
Proper engineering/technical standards & 2.14 & $\checkmark$ \\
Health and radiological safety impacts and benefits & 2.14 & \\
Human health impacts & 2.10 & $\checkmark$ \\
Health physics & 2.05 & $\checkmark$ \\
Geohydrology & 1.95 & $\checkmark$ \\
Aquatic resources & 1.95 & $\checkmark$ \\
Cultural diversity issues & 1.90 & \\
Soils and minerals & 1.90 & $\checkmark$ \\
Packaging issues & 1.86 & \\
Wildlife management & 1.86 & \\
Geophysics & 1.81 & \\
Nuclear engineering & 1.76 & \\
Cartography & 1.76 & $\checkmark$ \\
Geomapping, remote sensing techniques & 1.67 & $\checkmark$ \\
\hline
\end{tabular}

a Average score is based on a 4-point scale: $1=$ no/little knowledge; $2=$ heard of/familiar with; 3 = proficient; 4 = expert. 
TABLE C.5 10 Highest Average Scores on Regulatory Knowledge

\begin{tabular}{|c|c|c|c|}
\hline General Skill & Average $^{\mathrm{a}}$ & $\begin{array}{l}\text { Wide Range } \\
\text { of Abilities }\end{array}$ & $\begin{array}{l}\text { Common } \\
\text { Skills }\end{array}$ \\
\hline $\begin{array}{l}\text { Comprehensive Environmental Response, Compensation } \\
\text { and Liability Act }\end{array}$ & 2.90 & $\checkmark$ & \\
\hline National Environmental Policy Act & 2.86 & $\checkmark$ & \\
\hline Resource Conservation and Recovery Act & 2.81 & $\checkmark$ & \\
\hline $\begin{array}{l}\text { DOE Order } 5400.4 \text { Comprehensive Environmental } \\
\text { Response, Compensation and Liability Act Requirements }\end{array}$ & 2.71 & $\checkmark$ & \\
\hline $\begin{array}{l}\text { DOE Order 5440.1E National Environmental Policy Act } \\
\text { Compliance Program }\end{array}$ & 2.52 & $\checkmark$ & \\
\hline Occupational Safety and Health Act & 2.33 & $\checkmark$ & \\
\hline Clean Water Act, as amended & 2.25 & & \\
\hline $\begin{array}{l}\text { DOE Order 5000.3B Occurrence Reporting and Processing } \\
\text { of Operations Information }\end{array}$ & 2.24 & & \\
\hline Emergency Planning and Community Right-to-Know Act & 2.19 & $\checkmark$ & \\
\hline Toxic Substances Control Act & 2.19 & $\checkmark$ & \\
\hline $\begin{array}{l}\text { DOE Order } 4700.1 \text { Project Management System and } \\
\text { Associated Notices }\end{array}$ & 2.15 & $\checkmark$ & \\
\hline
\end{tabular}

a Average score is based on a 4-point scale: $1=$ no/little knowledge; 2 = heard of/familiar with; $3=$ proficient; $4=$ expert. 
TABLE C.6 10 Lowest Average Scores on Regulatory Knowledge

\begin{tabular}{|c|c|}
\hline General Skill & Average $^{a}$ \\
\hline DOE Order 5482.1B Environment, Safety, and Health Appraisal Program & 1.50 \\
\hline DOE Order 1500.3 Foreign Travel Authorization & 1.48 \\
\hline $\begin{array}{l}\text { DOE Order } 5484.1 \text { Environmental Protection, Safety, and Health Protection } \\
\text { Information Reporting Requirements }\end{array}$ & 1.48 \\
\hline DOE Order 5500.1B Emergency Management System & 1.48 \\
\hline $\begin{array}{l}\text { DOE Order 5500.2B Emergency Categories, Classes, and Notification and Reporting } \\
\text { Requirements }\end{array}$ & 1.48 \\
\hline Coastal Zone Management Act & 1.43 \\
\hline Fish and Wildlife Coordination Act & 1.43 \\
\hline DOE Order 4300.1C Real Property Management & 1.43 \\
\hline Executive Order 12088 Federal Compliance with Pollution Control Standards & 1.43 \\
\hline $\begin{array}{l}\text { Executive Order } 12780 \text { Federal Agency Recycling and the Council on Federal Recycling } \\
\text { and Procurement Policy }\end{array}$ & 1.43 \\
\hline Marine Mammal Protection Act & 1.40 \\
\hline Noise Control Act & 1.38 \\
\hline Prime Farmland Act & 1.38 \\
\hline Archaeological Resources Protection Act & 1.33 \\
\hline National Wildlife Refuge System Administration Act & 1.33 \\
\hline $\begin{array}{l}\text { DOE Order } 5480.15 \text { Department of Energy Laboratory Accreditation Program for } \\
\text { Personnel Dosimetry. }\end{array}$ & 1.33 \\
\hline Executive Order 11514 Protection and Enhancement of Environmental Quality & 1.33 \\
\hline Executive Order 11593 Protection and Enhancement of the Cultural Environment & 1.33 \\
\hline $\begin{array}{l}\text { Executive Order } 12777 \text { Implementation of Section } 311 \text { of the Federal Water Pollution } \\
\text { Control Act of 1972, as amended, and the Oil Pollution Act of } 1990\end{array}$ & 1.33 \\
\hline Rivers and Harbors Act & 1.29 \\
\hline DOE Order 4320.1B Site Development Planning & 1.29 \\
\hline Marine Protection, Research, and Sanctuaries Act & 1.24 \\
\hline Oil Pollution Act & 1.24 \\
\hline $\begin{array}{l}\text { Executive Order } 11738 \text { Providing for the Administration of the Clean Air Act and the } \\
\text { Federal Water Pollution Control Act with Respect to Federal Contracts, Grants, or Loans }\end{array}$ & 1.24 \\
\hline American Indian Religious Freedom Act & 1.19 \\
\hline International Maritime Act & 1.19 \\
\hline Migratory Bird Treaty Act & 1.19 \\
\hline Wild Horse and Burros Act & 1.19 \\
\hline Executive Order 11989 Off-Road Vehicles on Public Lands & 1.19 \\
\hline Native American Graves Protection and Repatriation Act & 1.14 \\
\hline Noxious Weed Act & 1.14 \\
\hline Repatriation Act & 1.14 \\
\hline Executive Order 11987 Exotic Organisms & 1.14 \\
\hline
\end{tabular}

a Average score is based on a 4-point scale: $1=$ no/little knowledge; 2 = heard of/familiar with; 3 = proficient; 4 = expert. 